MASS-YIELD VARIATIONS IN THE THERMAL AND EPI-THERMAL FISSIONS OF ${ }^{239} \mathrm{Pu}$ 


\title{
MASS-YIELD VARIATIONS IN THE THERMAL AND EPI-THERMAL FISSIONS OF ${ }^{239} \mathrm{Pu}$
}

\author{
By \\ SOO-LOONG TONG, B.SC.
}

\begin{abstract}
A Thesis
Submitted to the School of Graduate Studies

in Partial Fulfilment of the Requirements

for the Degree

Doctor of Philosophy
\end{abstract}

McMaster University

September, 1971 
TITLE: Mass-yield Variations in the Thermal and Epi-thermal Fissions of $239 \mathrm{Pu}$

AUTHOR: Soo-Loong Tong, B.Sc. (Nanyang University, Singapore)

SUPERVISOR: Dr. K. Fritze

NUMBER OF PAGES: $\quad x i, 121$

ABSTRACT :

Some cumulative mass-yield ratios for thermal to epithermal neutron fissions of ${ }^{239} \mathrm{Pu}$ have been measured by nondestructive $\mathrm{Ge}(\mathrm{Li})$ spectrometric and radiochemical

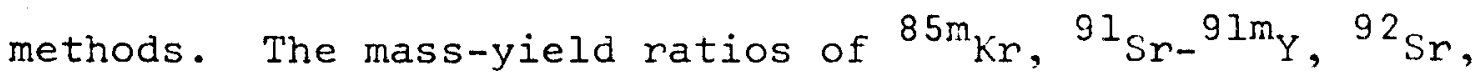
${ }^{97} \mathrm{Nb}-{ }^{97} \mathrm{Zr},{ }^{99 \mathrm{~m}_{\mathrm{Tc}},}{ }^{103} \mathrm{Ru},{ }^{105} \mathrm{Ru},{ }^{13 I_{\mathrm{I}},}{ }^{132} \mathrm{Te}-{ }^{132} \mathrm{I},{ }^{13} 3_{\mathrm{I}}$, ${ }^{135} \mathrm{I}-{ }^{135} \mathrm{Xe},{ }^{140} \mathrm{Ba}-{ }^{140} \mathrm{La},{ }^{143} \mathrm{Ce}$ and ${ }^{147} \mathrm{Nd}$ for both thermal to epi-Cd and thermal to epi-Sm fissions did not show any significant fluctuations. The yields of ${ }^{72} \mathrm{Zn}$ and ${ }^{166}$ Dy${ }^{166} 6_{\mathrm{HO}}$ in epi-Sm fission were found to be $30 \pm 2 \%$ lower than in the fission caused by thermal neutrons. Similarly the yields of ${ }^{115} \mathrm{Cd}$ and ${ }^{112} \mathrm{Pd}$ were found to be lower by $46 \pm 2 \%$ and $24 \pm 3 \%$ respectively. The 77 mass chain, measured as both ${ }^{77} \mathrm{Ge}$ and ${ }^{77} \mathrm{As}$, showed only a very slight, if any, decrease for epi-Cd and epi-Sm fissions as compared with thermal neutron fissions.

The results are consistent with the two-mode-offission hypothesis. An attempt has also been made to 
correlate the observations with the effect of the spin states of the fissioning nucleus at the saddle point. 


\section{ACKNOWLEDGEMENTS}

The author wishes to express his appreciation for the guidance and encouragement of his research director, Professor K. Fritze, throughout the course of this work. The author is indebted to the members of his supervisory committee, Professors R. H. Tomlinson and T. J. Kennett, for their helpful suggestions.

Financial support by the National Research Council of Canada and the Department of Chemistry, McMaster University is gratefuliy acknowledged. 
TABLE OF CONTENTS

Page

CHAPTER 1. INTRODUCTION

A. General Introduction 1

B. The Nature of Fission 10

C. Radiochemical Studies of Cumulative Massyield Distribution $\quad 14$

1. Radiochemistry 14

2. Cumulative Mass-yield Distribution 15

D. The Two-mode-of-fission Hypothesis 19

E. The Effect of the Spin States of the Compound Nucleus at the Saddle Point 25

CHAPTER 2. EXPERIMENTAL PROCEDURE

A. Introduction

B. The ${ }^{239}$ Pu Sample Preparation and Irradiation 34

C. Ge(Li) Gamma-ray Spectroscopy 38

D. Radiochemistry 39

1. Carrier solution preparations 39

2. Separation of ${ }^{239} \mathrm{Pu}$ from fission products

3. Separation and purification of $72 \mathrm{Zn}$, ${ }^{112} \mathrm{Pd}$ and ${ }^{115} \mathrm{Cd}$

4. Separation and purification of ${ }^{77} \mathrm{Ge} \quad 46$

5. Separation and purification of ${ }^{77} \mathrm{As} \quad 48$ 


\section{TABLE OF CONTENTS (Continued)}

Page

6. Separation and purification of ${ }^{166_{\text {Ho }}} 49$

CHAPTER 3. EXPERIMENTAL RESULTS

A. Treatment of Gamma-ray Spectra

B. The Thermal to epi-Cd and Thermal to epi-Sm Mass-yield Ratios of Products from Asymmetric Fission

C. The Thermal to epi-Sm Mass-yield Ratios of ${ }^{72} \mathrm{Zn},{ }^{112} \mathrm{Pd}$ and ${ }^{115} \mathrm{Cd}$

D. The Thermal to epi-Cd and Thermal to epiSm Mass-yield Ratios of ${ }^{77} \mathrm{Ge}$ and ${ }^{77} \mathrm{As} \quad 74$

E. The Thermal to epi-Sm Mass-yield Ratios of ${ }^{166} \mathrm{Dy}-{ }^{166} \mathrm{HO}$

F. Cadmium Ratios and Fast Neutron Fission Contribution

\section{CHAPTER 4. DISCUSSION}

A. Relative Fission Rates of the Low-energy

Resonances in the Thermal, epi-Cd and epi-Sm Fissions of ${ }^{239} \mathrm{Pu}$

B. Mass-yield Variations for Asymmetric Fissions

C. Mass-yield Variations for Symmetric and Very Asymmetric Fission

1. Interpretation in terms of the twomode of fission hypothesis 
TABLE OF CONTENTS (Continued)

Page

2. Interpretation in terms of the effect of the transition states of the compound nucleus at the saddle point 102

D. Conclusion

106

APPENDIX A. Correction for Neutron Capture Effect

107

APPENDIX B. Calculation of the Fractional Chain Yield 111

BIBLIOGRAPHY

113 


\section{LIST OF FIGURES}

Number

Page

1 Schematic map of the liquid-drop distortion

energy as function of two deformation

coordinates

2 Saddle-point shapes for various values

of the fissionability parameter $\mathrm{x}$

Schematic illustrations of single-humped

and double-humped fission barriers

Typical fission mass-yield distribution

curves

Dependence of fragment mass-yield on

excitation energy and target atomic number

Branching scheme of the $A=77$ mass chain

Distribution coefficient of $\mathrm{Pu}(\mathrm{IV})$ on

Dowex-1 in nitric acid

8

The effect of $\mathrm{pH}$ on elution of tracer

lanthanides from Dowex-50 cation exchange

resin with $\alpha-H I B$

Distribution coefficients of the lanthanides in Ambelite IRA-400 in EDTA form

Elution curve of holmium and ${ }^{165}$ Dy tracer

from Dowex-50 cation exchange column with $\alpha-H I B$ as eluant 


\section{LIST OF FIGURES (Continued)}

Number

Page

11 Elution curve of holmium and ${ }^{90} \mathrm{Y}$ tracer

12 Gross fission-product $\mathrm{Ge}(\mathrm{Li})$ spectrum for ${ }^{239} \mathrm{Pu}(\mathrm{n}, \mathrm{f})$ ( 20 hours after irradiation)

13 Gross fission-product $\mathrm{Ge}(\mathrm{Li})$ spectrum for ${ }^{239} \mathrm{Pu}(\mathrm{n}, \mathrm{f})$ (100 hours after irradiation) 64

Gamma-ray spectrum of ${ }^{72} \mathrm{Zn}$

Gamma-ray spectrum of ${ }^{112} \mathrm{Pd}-{ }^{112} \mathrm{Ag}$

Gamma-ray spectrum of ${ }^{115} \mathrm{Cd}-115 \mathrm{~m}$ In

72

17

Gamma-ray spectrum of ${ }^{77} \mathrm{Ge}$

75

18

Gamma-ray spectrum of ${ }^{77}$ As

76

19

Gamma-ray spectrum of ${ }^{166_{\text {Ho }}}$

78

20

$\mathrm{Ge}(\mathrm{Li})$ gamma-ray spectrum of epi-Sm

neutron irradiated ${ }^{232} \mathrm{Th}$

21

The reactor neutron spectrum

Decomposition of fission mass-yield

curve into two components 


\section{LIST OF TABLES}

1. Direct and indirect assignments of ${ }^{235} \mathrm{U} \quad$ Page resonance $\operatorname{spin}(\mathrm{J})$

2. Direct and indirect assignments of ${ }^{239} \mathrm{Pu}$ resonance $\operatorname{spin}(\mathrm{J})$

3. Distribution coefficients of plutonium on 42. strong base anion exchange resin in $\mathrm{HCl}$

4. Separation factors of rare earth elements on Dowex-50 with ammonium $\alpha$-hydroxyisobutyrate as eluant

5. Atomic abundances of rare earth elements 60

6. Prominent gamma-rays in gross fission products 65 Ge $(I i)$ spectrum

7. Activity ratios of fission products for thermal 67,68 to epi-thermal fission of ${ }^{239} \mathrm{Pu}$ from nondestructive Ge $(L i)$ measurements

8. Measured and normalized mass-yield ratios for thermal to epi-thermal fission of ${ }^{239} \mathrm{Pu}\left({ }^{72} \mathrm{zn}\right.$, $112 \mathrm{Pd}$ and ${ }^{115} \mathrm{Cd}$ )

9. Measured and normalized mass-yield ratios for thermal to epi-thermal fission of ${ }^{239} \mathrm{Pu}$ $\left({ }^{77} \mathrm{Ge}\right.$ and $\left.{ }^{77} \mathrm{As}\right)$

10. Measured and normalized mass-yield ratios for thermal to epi-thermal fission of ${ }^{239} \mathrm{Pu}$ 


\section{LIST OF TABLES (Continued)}

Page

11. Fast-neutron fission contribution in ${ }^{239} \mathrm{Pu}(\mathrm{n}, \mathrm{f}) \quad 87$

12. Parameters of prominent neutron capture 89 resonances of Samarium

13. Relative fission rates of low energy resonances 90 in the thermal, epi-cd and epi-sm fission of ${ }^{239} \mathrm{Pu}$

14. Activity ratios of fission products for thermal 93 to epi-cd fission of ${ }^{235_{U}}$ from nondestructive Ge (Li) measurements

15. Summary of the mass-yield ratios for thermal to 96 epi-thermal fission of ${ }^{239} \mathrm{Pu}$ 
CHAPTER 1

INTRODUCTION

\section{A. General Introduction}

The splitting of the atomic nucleus was first discovered by Hahn and Strassmann (1) in 1939 through careful and unambiguous radiochemical identification of barium and lanthanum activities from neutron-bombarded uranium targets. The term 'fission' was proposed by Meitner and Frisch (2) and they gave a qualitative interpretation of the process as the division of an excited uranium nucleus into two fragments of intermediary weights, in analogy with a charged liquid drop in which the repulsive Coulomb forces compete with the attractive surface tension. This was followed by the first theoretical paper of Bohr and Wheeler (3) which described the mechanism of fission in terms of the liquid drop model.

Intensive experimental efforts made during the war

years (4) have revealed many of the fission properties as we know them today. Advances in radiochemistry and, particularly in the techniques of nuclear physics in the past two decades have added many detailed features concerning the energetics, and mass and charge distributions of this phenomemon (see reviews (5), (6) and (7)). 
We list below a few of the more remarkable features for common fissile materials such as ${ }^{235} \mathrm{U}$ and ${ }^{239} \mathrm{Pu}$. Those relevant to the present work are discussed in the subsequent sections. Regarding the mass-yield distribution, the asymmetric double-humped nature which indicates the much higher probability for fission into two unequal fragments (of masses $\sim 100$ and 140 ) than into two nearly equal fragments ( 600 to 1 for thermal neutron induced fission of ${ }^{235} \mathrm{U}$ ), has been well established and is still puzzling.

Charge distribution data have been correlated and predicted in a strictly empirical way more successfully than the theoretically oriented postulates. There are two aspects to the empirical correlations: First, the most probable charge division for a given mass split can be summarized by the equal charge displacement hypothesis of Glendenin et al. (8). The hypothesis states that the most probable charges ( $\mathrm{pp}$ ) for one fission fragment and for its complementary fragment lie an equal.number of units away from the beta stability. Second, the distribution in fragment charge for a given mass chain can be approximated by a Gaussian, $\exp \left(-\left(z-z_{p}\right)^{2} / c\right)$, where $c=0.80 \pm$ 0.14 according to a recent paper of walhl et al. (9).

The major part of the fission energy release (180-200MeV) is in the form of kinetic energy of the fragments ( 150MeV) with the remainder as fragment deformation and intrinsic excitation energies. The total kinetic energy does not show a linear variation with the fragment mass ratio but rather a maximum 
occurs at a mass ratio of 1.2 to 1.3 instead of 1.0 . The excitation energy is dissipated mostly in neutron emission after the fragments are separated. The dependence of the neutron yield on fragment mass is rather striking. For near symmetric fission, the lighter fragment has a higher neutron yield than the heavier fragment. The effect is reversed for very asymmetric fission hence giving rise to the famous sawtooth shape for the prompt-neutron yield distribution. Theoretical developments on the other hand are briefly introduced below.

The fission of heavy elements such as uranium and plutonium is a many-body problem. An accurate solution of the many-body Schroedinger equation, in principle, will yield all aspects of fission. But this is still formidable in practice because of the limited capacity of even the most advanced computing machines. Various mathematical and physical approximations (models) have been proposed and useful information extracted and compared with experimental observations.

The most extensively studied theory is the liquid drop model which was originated from the monumental work of Bohr and wheeler (3). In this model, the potential energy of the charged liquid drop can be split into two terms, the electrostatic energy which pulls the drop apart, and the surface energy which holds it together. As a measure of the fissionability, Bohr and wheeler (3) introduced the 
parameter $x=E_{C}^{0} / 2 E_{s}^{0}$ where $E_{C}^{0}$ and $E_{s}^{0}$ are the Coulomb and surface energies of the uniformly charged spherical drop with a sharp surface. This is equivalent to $x=\left(z^{2} / A\right) /\left(z^{2} / A\right)$ critical where $\left(\mathrm{z}^{2} / \mathrm{A}\right)_{\text {critical }}=50.13$ $x \geq 1$ are unstable towards fission. The division of the drop is described as the transition from a nearly spherical configuration of low energy to another one representing the separated fragments. A typical contour map of the potential energy surface as function of the deformation coordinates, in the liquid drop model calculation, is shown in Fig. 1. The transition is pictured to pass through the saddle point and the configuration at this point is expected to be of special importance in fission theory. The statics of the model as a result of analytic and numerical development mainly from the works of Cohen and Swiatecki (10), and Strutinskii, Lyashchenko and Popov (11), is rather well understood. The saddle point shapes of nuclei with different fissionability parameters $(x)$ are depicted in Fig. 2.

Dynamic consideration was first introduced by Hill and Wheeler (12) in tracing out the shapes of the fissioning nucleus descending from the saddle point to the stage of scission. In recent works, Nix (13) has developed further the dynamics in addition to the statics, statistical mechanics and also some aspects of the quantum mechanics in studying the fission of the nucleus idealized as a nonviscous irrotational 


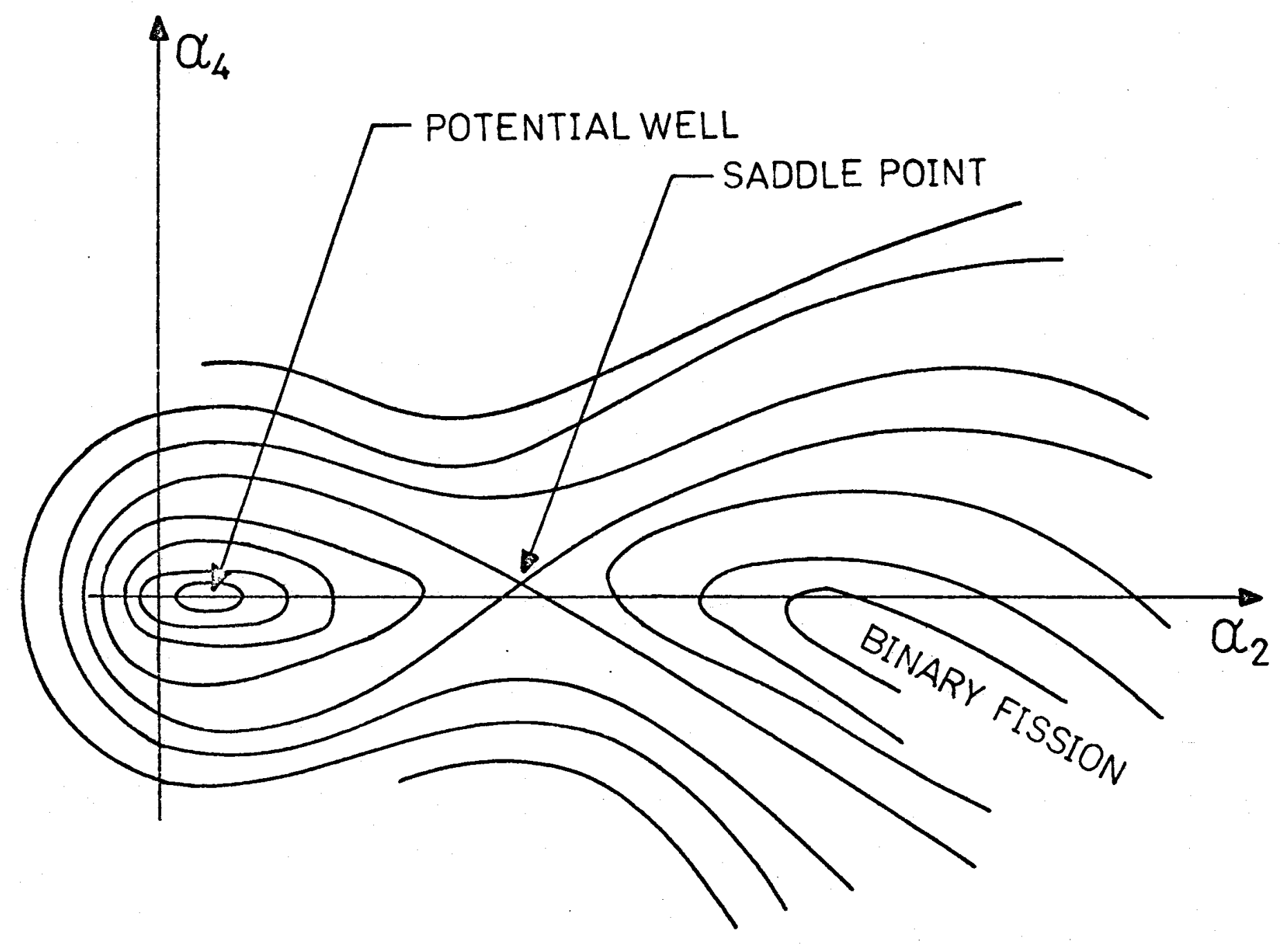

Fig. 1. Schematic Map of the Liquid-drop Distortion Energy as Function of Two Deformation Coordinates. 


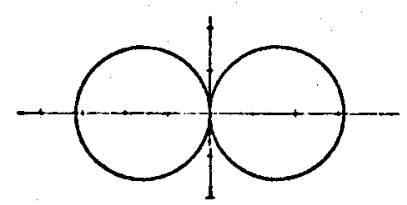

$x=0.0$

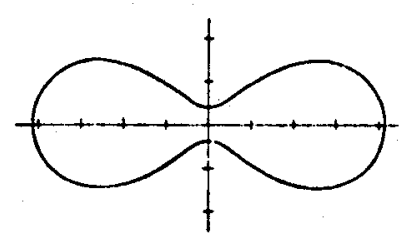

$x=0.5$

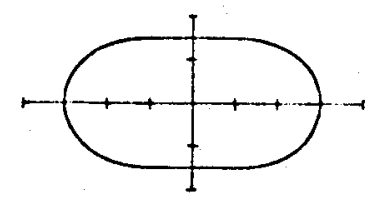

$\mathbf{x}=0.8$

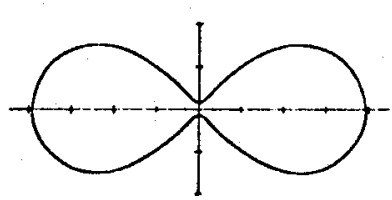

$x=0.3$

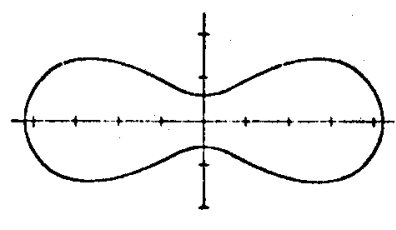

$x=0.6$

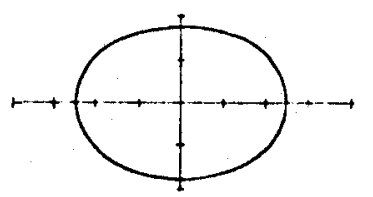

$x=0.9$

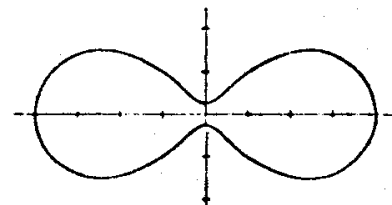

$x=0.4$

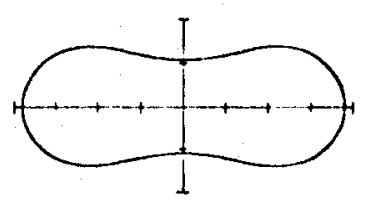

$x=0.7$

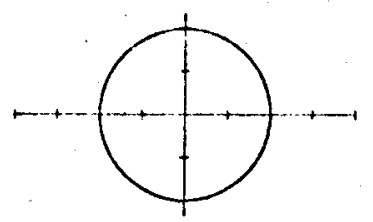

$x=1.0$

Fig. 2. Saddle-point Shapes for Various Values of the Fissionability Parameter $x$ (10). 
liquid drop. Though such a calculation is still incapable of accounting for fission of heavy elements (heavier than about radium) at low excitation energy, it did reproduce the experimental fission-fragment mass and energy distributions for fission of heavy elements at high excitation energy $(>40 \mathrm{MeV})$ and mediumweight nuclei (between about silver and radium) at all excitation energies.

The inclusion of a shell correction term in the liquid drop model calculation by strutinskii (14) which gave rise to a double-humped fission barrier instead of the conventional single-peaked shape (see Fig. 3) has been the most remarkable event in this field for the past few years. The doublehumped fission barrier provides satisfactory explanation for some anomalous observations of fission, particularly in the observed fission nuclei isomerism, broad resonance structures in the fission excitation functions, etc. (15)

So far, the liquid drop model approach still fails to explain the most striking feature of fission of the heavy elements such as ${ }^{235} \mathrm{U}$ and ${ }^{239} \mathrm{Pu}$, namely the asymmetric fission into two unequal fragments being much more probable than symmetric fission into two nearly equal fragments.

The statistical model proposed by P. Fong, (16) was the only one that has given detailed quantitative calculations and he obtained a mass-yield distribution curve for thermal neutron induced fission of ${ }^{235} \mathrm{U}$ which agreed well with the experimental data. Fong assumed that fission is a slow process 


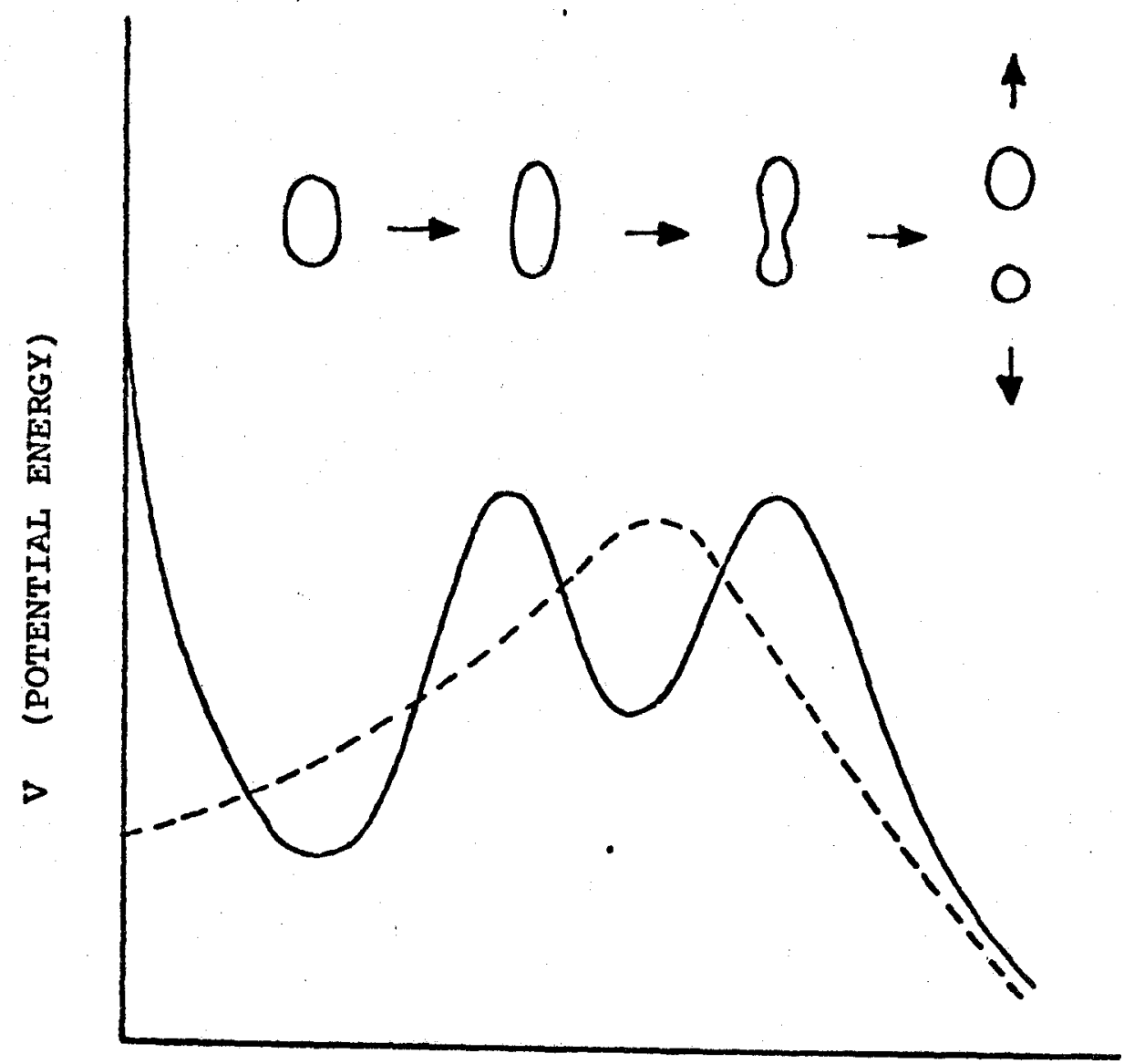

\section{B (DEFORMATION)}

Fig. 3. Schematic illustrations of single-humped (dashed line) and double-humped (solid line) fission barriers. The transition in the shape of the nucleus is schematically represented in the upper part of the figure. 
so that equilibrium is established instantaneously at or near the point of scission. The statistical quantities such as the mass distribution, are related to the total energy of excitation of the two nascent fragments at the point of scission as well as to the level density. The nuclear level density was examined empirically with shell effects taken into account. Though this model appears to be complete and free of arbitrary parameters, later application seems to indicate that it does not reproduce the experimental data satisfactorily. When Perring and storey ${ }^{(17)}$ applied the model to the thermal fission of ${ }^{239} \mathrm{Pu}$, a four-humped mass-yield distribution was obtained. As Fong pointed out more recently, (18) the application of this model is sensitive to the input parameters including the nuclear masses, the deformation parameters, the level density function etc. Therefore more reliable information on them should be available before the model may be tested conclusively.

Other models which emphasize the nuclear structure of the primary fragments at the point of scission as determining factor on fission fragment distributions include the works of Brunnerand Paul (19), Thomas and Vandenbosch, (20), Ramanna et al. (21) among others.

Another class of theory basically different from the statistical model is the adiabatic model (22). According to this model, the collective deformation is assumed to be slow compared with the typical period of nucleon motion so that 
particle motion can be adjusted adiabatically to the collective motion of the nuclear surface at the stage of transition from saddle to scission. The practical nonviscous liquid drop model calculation as pointed out by Swiatecki (23), and the channel theory of A. Bohr (24) fall into this class. Bohr's theory which suggests memory of the saddle-point properties in the fission process has enjoyed great success especially in accounting for fission fragment angular anisotropies among other phenomena. The cluster model of Faissner and wildermuth (25) also suggests that the mass and charge distribution may be determined by nuclear configuration already existing at the saddle point.

The assumption of equilibrium at or near the point of scission and the principle of memory of the saddle-point properties in fission process are contradictory to each other but both achieved a remarkable measure of success. Griffin (26) proposed in the Second Symposium on the Physics and Chemistry of Fission (1969), that rapid collective motion during the late stages of fission may lead to memory of the saddlepoint properties but not realizing the equilibrium in the mean time. An appreciable amount of contributions in this regard can also be found in the same symposium. However, no theory as yet has been able to account for all aspects of the complicated phenomenon.

B. The Nature of Fission 
peting with other modes of decay (emission of $\alpha, \beta, \gamma$ or other nuclear particles) whenever the nucleus is excited with sufficient energy to overcome the fission energy barrier. In those cases where the fission barrier is sufficiently low, tunnelling from the ground state leads to spontaneous fission.

Excitation of the nucleus may be through compound nucleus interaction with photons, neutrons, positively charged particles, mesons etc., or through direct interaction such as $(\alpha, p),(\alpha, \alpha)$ etc.. Many targets from about silver up to the heaviest man-made elements have been reported to undergo fission. The typical and most extensively studied are the low energy neutron fissions of ${ }^{233} \mathrm{U},{ }^{235} \mathrm{U}$ and ${ }^{239} \mathrm{Pu}$ and fast neutron fissions of 232 Th and ${ }^{238} \mathrm{U}$.

It is generally assumed that the fission process takes place in sequential stages, though no definite boundaries have been set between stages. The following discussion mainly refers to typical low energy neutron induced fission. However, though the lifetime for fission is related to the excitation energy, the fission mechanism at different excitation energies is in many ways quite similar.

The first stage is the formation of the compound nucleus. The excitation energy in the form of the binding energy and kinetic energy of the incident neutron is shared among the nucleons.

The second stage is the crossing of the saddle point. The surface energy increases initially with deformation but the 
Coulomb energy decreases. The Coulomb energy decreases faster than the increase in surface energy for large deformation so that at intermediate deformations there exist some transition states. They are usually designated as fission channels, each with one or a few modes of motion.

The passige over the deformed saddle shape is the ratedetermining step and the time required can be estimated from the observed sizes of the fission widths of resonances as about $10^{-14}$ to $10^{-12}$ se:c.

The next stage is the transition from the saddle shape to the point of sicission, which is defined as the point beyond which no nuclear interaction between the fragments exists. The time taken has been estimated to be $\sim 10^{-21}-10^{-20} \mathrm{sec}$ ( ${ }^{-28)}$. When the scission point has been reached, the fragment mass and charge distr:butions are established.

The scission process may involve a violent snapping action, permittiug the release of some of the prompt neutrons, and sometimes a light charged particle, most probably an alpha-particle in between the two separating fragments (27). This is known as ternary fission; typically the most probable a-ternary fission is about one four-hundredth of the binary fission for thermal neutron-induced fission of ${ }^{235} \mathrm{U}$ (28). After scission, the nascent fragments, usually called primary fission fragments, are highly deformed in shape and they possess little kinetic energy at the first instant. 
The fragments accelerate apart under the long range Coulomb interaction, taking approximately $10^{-20} \mathrm{sec}$ to reach $90 \%$ of the final kinetic energy, in the range of 50 to $105 \mathrm{MeV}$. Fission characteristics of interest that can be measured at this stage include primary fission fragment mass, kinetic energy and angular distributions.

At a later stage of the separation, the highly deformed fragments reorient themselves and emit most or all of the prompt neutrons, prompt fission gamma-rays and conversion-electron radiations. The times required for the prompt neutron and gamma-emission are $10^{-15}-10^{-18}$ and $\sim 10^{-11} \mathrm{sec}$ respectively. The prompt neutron and gamma emission yields as a function of fragment mass and the kinetic energy distribution provide useful information about the fission energetics.

Finally, the primary fission products (to be distinguished from primary fission fragments before neutron and gamma emissions) being far removed from the beta stability line will undergo sequential beta decays,accompanied by delayed gamma and conversion-electron, and characteristic $\mathrm{x}$-rays emissions. This process is slow and has a life-time $\geqslant 10^{-3}$ sec. Delayed neutron emission which is of crucial importance to nuclear reactor design arises following beta-decays of some primary fission products which lead to highly excited intrinsic states where neutron emission competes with further $\beta$ or $\gamma$-deexcitation.

If in a given mass chain the yields of nuclides with 
different atomic number were measured before any beta decay, they are known as the independent yields and reflect the charge distribution in the process of fission.

After sequential beta decays, the yield of the end product is called the cumulative yield of the mass chain. The cumulative mass-yield distribution is also expected to hinge on the nature of the mass splitting mechanism.

Since radiochemical studies of the cumulative massyield is of prime concern to the present work, the following is contributed as a reyiew of this field.

C. The Radiochemical Studies of Cumulative Mass-Yield Distribution

1. Radiochemical method

The common binary fission yields a spectrum of fission products of masses ranging from $\sim 70$ to $\sim 170$. Cumulative mass yield of a specific nuclide may be determined by chemical separation at the time all its precursors have undergone sequential beta decays, or when the parent and daughters are in a state of transient equilibrium.

At the beginning, a measured amount of the nonradioactive element, known as carrier of the specific nuclide of interest is added to the solution of the fissile material and its fission products. It is essential that isotopic 
exchange is completed between the stable and radioactive isotopes of the element. Conventional analytical procedure are then employed to separate the element from the fissile material and the gross fission products in a state of radiochemical purity. The beta or gamma radiations of the sample are measured with radio counting instruments to identify the isotope and determine the amount of the radioactive species. The chemical yield which was determined by some quantitative method should equal the percentage recovery of the isotope under investigation. Hence the fission yield of the specific isotope can be calculated from the counting data, the chemical yield and the known number of fission of the sample.

\section{Cumulative mass-yield distribution}

The mass-yield distributions of ${ }^{233} \mathrm{U},{ }^{235} \mathrm{U},{ }^{239} \mathrm{Pu},{ }^{232} \mathrm{Th}$, ${ }^{238} \mathrm{U}$ etc. have been well studied by this method. Fig. 4 shows the typical cumulative mass-yield distribution curves of the thermal neutron-induced fissions of ${ }^{235} \mathrm{U}$ and ${ }^{239} \mathrm{Pu}$, and the fast neutron fission of $232 \mathrm{Th}$.

For common fissile nuclides it is most striking that the mass-yield distributions are asymmetric in shape. The most probable heavy fragment mass number is 139, and the most probable light fragment mass number is found to vary from 94 to 100 , according to the mass number of the target nuclide. 


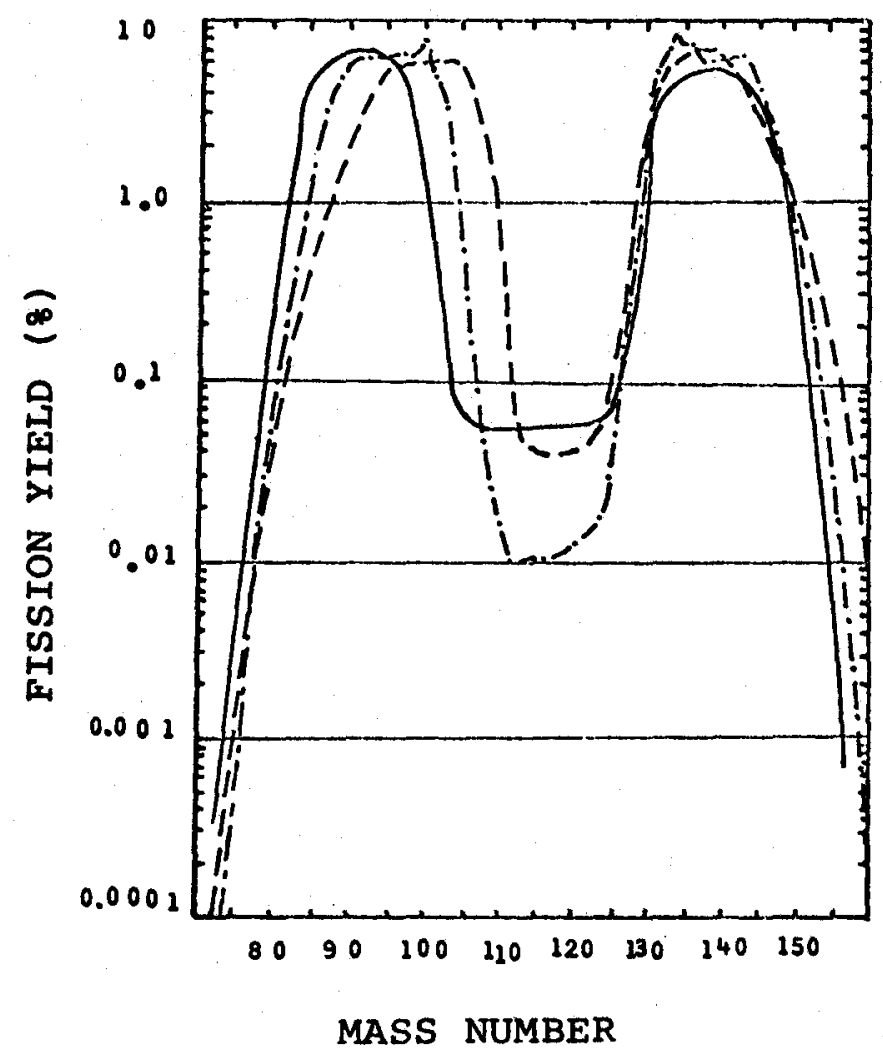

Fig. 4. Typical fission mass-yield distribution curves: for ${ }^{232}$ Th(fast $\left.n, f\right)$; _. _... for ${ }^{235} \mathrm{U}$ (thermal $n, f):--$ for ${ }^{239} \mathrm{Pu}($ thermal $n, f)$. 
The peak-to-valley mass-yield ratios for thermal neutron fissions ${ }^{235} \mathrm{U}$ and ${ }^{239} \mathrm{Pu}$ are 650 and 150 respectively.

Mass spectrometric techniques have also been applied in the studies of mass-yield distribution (30). Fine structure was found in the thermal neutron fission of ${ }^{235} \mathrm{U}$, for example, where the yield of ${ }^{134} \mathrm{Xe}$ was anomalously higher than expected from the smooth mass-yield distribution curve obtained by earlier radiochemical measurements.

The mass-yield distribution changes as a function of the mass number of the fissioning nucleus as well as a function of the excitation energy in the system. The general trends derived from experimental observed results can be summarized in Fig. 5 .

The fission of heavy $(z \geqslant 90)$ elements is predominantly asymmetric. The symmetric valley in the mass-yield distribution curve is virtually undectectable for spontaneous fission. But as the excitation energy of the fissioning nucleus is increased it begins to fill in. At high excitation, the situation is obscured because the later chanoe fission after emitting one or a few neutrons may contribute significantly. For excitation energies between the threshold and $\sim 20 \mathrm{MeV}$, very asymmetric fission yields in the wing regions of the mass yield curve increase in similar trend as the symmetric fission, according to the compilation of Katcoff (29) and also data reported by Levy et al. (31) and Borisova et al. (32). 


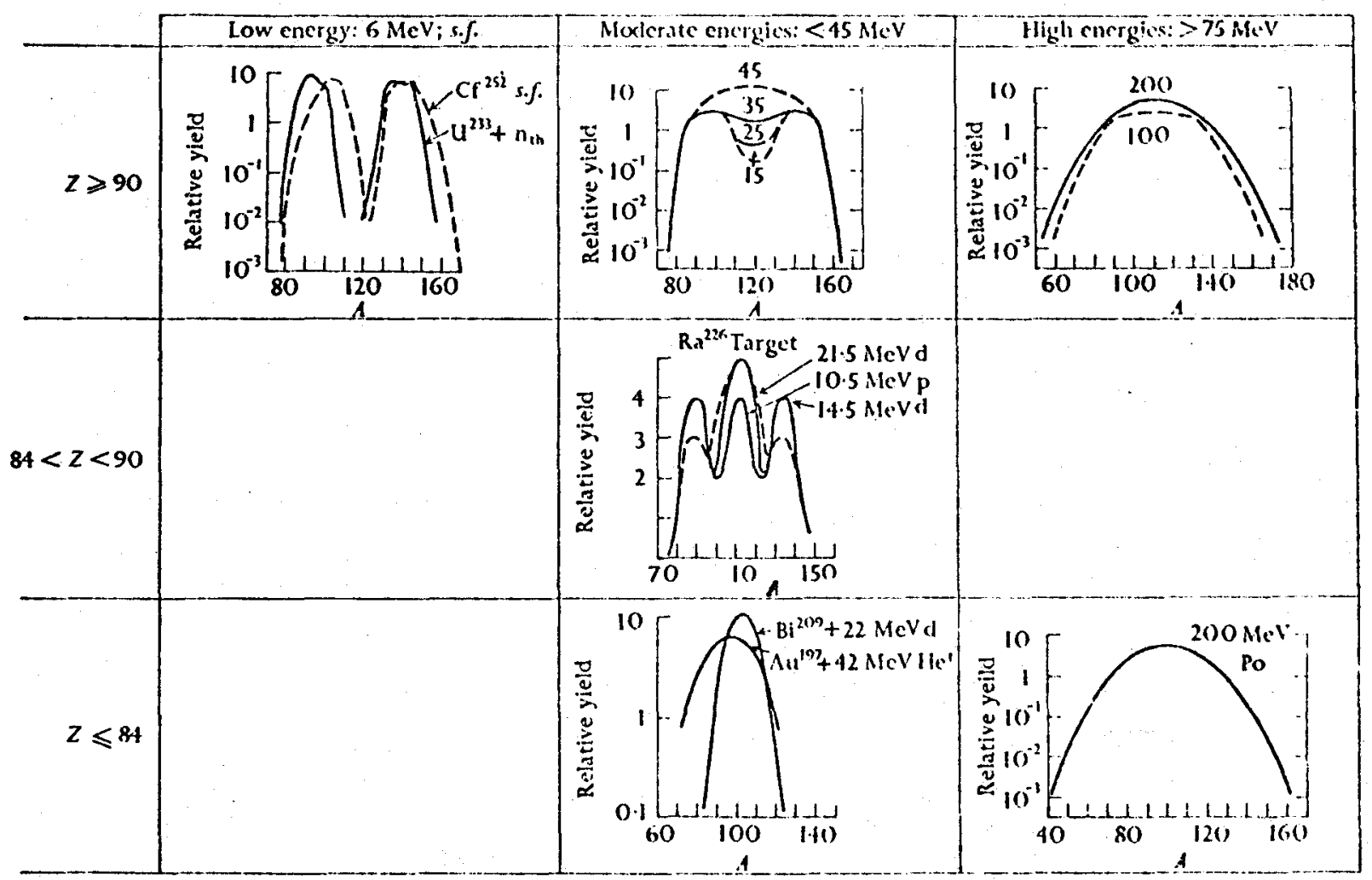

Fig. 5. The dependence of fragment mass-yield on excitation energy and the target atomic number. (From Wilets (22), 
The intermediate mass fissioners such as actinum and radium show a symmetric peak coexisting with the asymmetric peaks for near threshold fission

(33). Asymmetric fission diminishes at higher excitation.

The lighter fission elements $(z<84)$ exhibit predominantly symmetric fission distribution (33) which has also been found to broaden with increasing excitation energy

D. The Two-mode-of-fission Hypothesis

Speculative attempts have been made to account for the observed trends in mass yield distribution curves. One of these is the two-mode-of-fission hypothesis proposed by Turkevich and Niday $(34)$ in explaining a rather flat region observed in symmetric fission of ${ }^{232}$ Th induced by fast neutrons. According to this hypothesis, there are two independent modes for fission: one associated with symmetric and one with asymmetric mass distributions.

Fairhall et al. (33) defined the two-mode of fission on a different basis in view of the characteristic energy dependence and the lack of A (mass number) dependence of the symmetric fission in uranium and plutonium compound nuclei, coupled with the observation of the triple-peaked mass yield distribution curve of radium. They assumed that the symmetric mode corresponds to low excitation which occurs when fission takes place after the emission of one or more neutrons from the compound 
nucleus, and the asymmetric mode corresponds to primary fission of the compound nucleus at higher excitation energy. Decomposition of the mass yield curve into the two modes in several cases seems to be in satisfactory agreement with the hypothesis,e.g. ${ }^{233} \mathrm{U}$ fission with ${ }^{4} \mathrm{He}$ of energies ranging from $25.3 \mathrm{MeV}$ to $40.5 \mathrm{MeV}$ by Colby et al. (35) $232 \mathrm{Th}+$ pile neutrons by Iyer et al. (36) and also the work of Britt et al. (37) The latter authors were also able to correlate the character of total kinetic energy release as a function of mass split with the two fission modes.

Ford (38) developed a statistical test of the two-mode of fission hypothesis for experimentally observed mass-yield data in terms of linear algebra analysis. He regarded the two-mode hypothesis as implying that for a given target and projectile there are two and only two configurations for the compound nucleus that may result in fission. For each configuration there correspond many possible mass yields in different frequencies which can be represented by a vector as a whole. The total mass yield curve is a result of the combination of two linearly independent vectors. The variations of the mass yield distribution as a function of excitation energy is due to the change in proportions of the two configurations.

The statistical tests on fission yields from proton-induced fission of ${ }^{232} \mathrm{Th}$, proton-induced fission of ${ }^{238} \mathrm{U}, \alpha$ - particle induced fission of ${ }^{238} \mathrm{U}$, and deuteron-induced fission of natural uranium did give reasonably high levels of significance 
for accepting the hypothesis (38). Similar tests carried out by Levy et:al. (31) for neutron-induced fission of ${ }^{235} \mathrm{U}$ and ${ }^{238} \mathrm{U}$ showed general agreement except for thermal neutron induced fission. Continuing this work ${ }^{(39)}$ the authors found deviations at higher excitation energies for some mass numbers. It was thought that multi-chance fissions following emission of one or more neutrons might perturb the results. Fission before neutron emission is called first-chance, after the emission of one neutron is called second-chance, and so on. Hence in a later work, Ford and Leachman $(40)$ have taken this into account. The observed yields and the first-chance yields for ${ }^{236} \mathrm{U}$ compound nucleus, and also the observed yields for neutron-induced fission of ${ }^{232} \mathrm{Th}$ and ${ }^{238} \mathrm{U}$, were found in good agreement with the hypothesis. Using this method of analysis, Choppin and Meyer (41) also observed consistency with the two-mode hypothesis for their fission yield data of ${ }^{238} \mathrm{U}$ induced by $7.1,9.4$ and $12.0 \mathrm{MeV}$ protons.

A recent speculative interpretation of the mass yield distribution was made by Schmitt and Konecny (42). They suggested "two-component" instead of the two distinct modes hypothesis such that the symmetric and asymmetric components may arise from different "mechanisms"which may or may not involve separate saddle point states. On the basis of the experimental results of the fragment kinetics of the fission of ${ }^{226} \mathrm{Ra}$ with $13 \mathrm{MeV}$ protons, including fragment energies, masses 
and mass-energy distributions, they observed that the properties of the symmetric fission of radium were quantitatively consistent with the results of the liquid drop model calculations of $\mathrm{Nix}$ and Swiatecki (43) while the asymmetric fission could be ascribed to a mechanism determined by the nuclear structure properties (especially shell structures) of the fragments. For the symmetric component, the fragments were thought to be formed in softer, relatively highly deformed configurations characteristic of a liquid drop. While in the other component, the fragment shell effect seems to dominate, and the heavier fragments are formed in less highly deformed configurations.

The two-mode hypothesis derived from fission well above threshold energy has also been invoked in interpreting mass yield variations of fissions induced by resonance neutrons. Variations have been observed in the mass yield ratios of the asymmetric to symmetric fission fragments of ${ }^{233} \mathrm{U}$, and ${ }^{235} \mathrm{U}$ and ${ }^{239} \mathrm{Pu}$ for fissions with resonance neutrons. Radiochemical studies of such an effect are always measured in terms of the ' $R$ ' value which is defined as:

$$
R=\frac{\left(A_{\text {asym }} / A_{\text {sym }}\right)_{\text {resonance }}}{\left(A_{\text {asym }} / A_{\text {sym }}\right)_{\text {thermal }}}
$$

where $A$ is the corrected activity of a nuclide, subscript 'asym' refers to an asymmetric fission product (usually ${ }^{99} \mathrm{Mo}$ or ${ }^{140} \mathrm{Ba}$ ), and subscript 'sym' refers to a symmetric fission product 
$\left({ }^{115} \mathrm{Cd},{ }^{121} \mathrm{Sn},{ }^{113} \mathrm{Ag}\right.$, etc. $)$

The earliest work of the Los Alamos Radiochemistry

Group (44) measured the asymmetric to symmetric fission yield ratios for thermal to epi-cadmium neutron-induced fissions of ${ }^{235_{U}}$ and found a small but significant difference for the two types of fission. Later measurements of Faler and Tromp (45) on ${ }^{235} \mathrm{U}$ with monochromatic neutrons with energy below $2 \mathrm{eV}$ confirmed this effect.

More drastic changes were reported by Regier et al. (46) for the $0.297 \mathrm{eV}$ resonance fission of ${ }^{239} \mathrm{Pu}$, and "R" value of $\sim 2.4$ was found for ${ }^{115} \mathrm{Cd},{ }^{121} \mathrm{Sn}$ and ${ }^{125} \mathrm{sn}$. For all epi-samarium neutron fission, where the majority of the fission events is due to the $0.297 \mathrm{eV}$ resonance, effects of similar magnitude were also reported.

In the "wheel" experiments of Cowan et al. (47) for which atomic bomb explosions were used as neutron sources, fissions of ${ }^{235} \mathrm{U}$ and ${ }^{239} \mathrm{Pu}$ were studied with incident neutron energy ranging from tens to several hundred eV. Their results indicated that the asymmetric to symmetric yield ratios at different resonances can always be classified into two groups: one group was characterized by fission less symmetric than thermal-neutron fission and the other being more symmetric. Ford and Leachman ${ }^{(40)}$ applied statistical tests on the results of Cowan et al. and found no significant deviation from the two-mode hypothesis.

The idea of the variations in the mass yields of products 
from symmetric fission induced by neutrons in the resonance energy region as the competition of the two fission modes seems unable to account for the observation of Croall and Willis (48). They have found considerable increases in the yields of ${ }^{77}$ As and ${ }^{78}$ As accompanying decreases in the yields of products from symmetric fissions in the epi-Cd as compared with thermal neutron fissions of ${ }^{239} \mathrm{Pu}$. The contrary effect in 77 As and ${ }^{78} \mathrm{As}$ should be expected as we notice that for fission at well above threshold energy, the yields of products from very asymmetric fission on the far wings of the mass yield curve change in the same direction as the symmetric fission yields. Indeed a small but significant effect in ${ }^{72} \mathrm{zn}$ has been found in a study by Fritze (49) in which an $210 \%$ decrease in $72 \mathrm{zn}$ was associated with the decrease in ${ }^{115} \mathrm{Cd}$ for epi-Cd fission as compared with thermal neutron fission.

One of the purposes of this work was to throw some light on such contradictions. Regier et al. ${ }^{(46)}$ had shown that for all epi-sm neutron fission of ${ }^{239} \mathrm{Pu}$ the effect in the symmetric yields was almost comparable to the largest decrease observed for the $0.297 \mathrm{eV}$ resonance. One therefore could expect if there is any effect in the very asymmetric fission yields associated with that observed in the symmetric region for the $0.297 \mathrm{eV}$ resonance, study of the epi-Sm fission of ${ }^{239} \mathrm{Pu}$ would be as effective.

The fluctuations in the asymmetric to symmetric yield ratios which indicate that symmetric fission is more favorable 
in one group of resonances and less in the other has more often been related to the effect of the level structure of the compound nucleus at the saddle point, as we shall see in the next section.

E. The Effect of the Spin States of the Compound Nucleus at The Saddle Point on Fission Mass-Yield Distribution

Consideration of the effect of the spin states of the compound nucleus at the saddle point on post-scission characteristics was based on the channel theory introduced by A. Bohr in $1955^{(24)}$. According to Bohr, as the excited nucleus approaches the saddle point (for near threshold excitation), most of the excitation energy is converted to deformation energy. The nucleus is assumed to be "cold" at the saddle point and only a few widely separated quantum levels representing the motionare available. These levels are restricted to low energy vibrations and rotations similar to those which the compound system would exhibit near its ground state. Bohr assumed that some of the properties of the quantum states remain good quantum numbers, or otherwise "frozen-in", as the nucleus passes from the saddle point to the point of scission. Therefore one expects that these properties would give rise to some regularities in the post-scission observables. This assumption is basically the assumption of the adiabatic model and its validity depends on the duration of the transition from saddle point to scission. If it is so long that statistical equilibrium 
can be attained then the assumption of the statistical model prevails.

On the basis of Bohr's theory, it is possible to correlate the experimental fission fragment angular anisotropies with the level structure of the compound nucleus at the saddle point. It is generally accepted that the deformed nucleus at the saddle point is axially symmetric and it may be approximately described with a quantum number $\mathrm{k}$, defined as the component of the nuclear angular momentum along the symmetry axis of the nucleus itself. Bohr's theory predicts that the ultimate fission fragment direction would be closely related to the available $\mathrm{K}$ states in the transition state nucleus because the nuclear symmetry axis is assumed to be the axis along which the two fragments separate. There are numerous experimental works which have been consistently interpreted in terms of the theory (see (5), (6) and (7)). In fact, the general agreement between theory and experimental data on fission fragment angular distribution measurements has been so good that several authors have even used the theory to extract information on nuclear structure and the transition states for the saddle point nucleus.

Bohr's theory has been extended to a few other fission phenomena. Wheeler $(50)$ suggested that the mass yield ratios of asymmetric to symmetric fission products might differ from resonance to resonance for low energy neutron fissioners, depending on the spin states of the fissioning compound nucleus at the saddle point. The argument was based on a consideration 
of the symmetry characters of the wave function of the fissioning nucleus at the saddle point and assuming that (i) fission takes place through only a few lowest-lying fission channels, and (ii) the symmetry character is a constant of motion for the transition from saddle to scission. The type of symmetry character responsible is the octupole deformation which consists of the mass-asymmetry vibration (or sloshing mode) in which material is transferred from one end of the prolate body to the other, and the bending vibration mode.

The spin states for a compound nucleus formed by low energy (S-wave) neutrons incident on a target nucleus with spin and parity of $I^{\pi}$ can be either $(I+1 / 2)^{\pi}$ or $(I-1 / 2)^{\pi}$. The possible spin states $\mathrm{J}^{\pi}$ of the compound nuclei ${ }^{236} \mathrm{U}$ and ${ }^{240} \mathrm{Pu}$ formed by s-wave neutron absorption are $3^{-}$or $4^{-}$, and $\mathrm{O}^{+}$or $1^{+}$ respectively.

It is believed that for ${ }^{236} \mathrm{U}{ }^{(51)}$ the $3^{-}$levels proceed through a fully open saddle point channel with one quantum of of mass-asymmetry vibration (and rotation), for which symmetric fission will be forbidden. It may also proceed through a partially or fully open channel corresponding to bending vibration where symmetric fission is allowed. The latter is the only open channel possible for the $4^{-}$levels. Consequently, one expects the average mass yield ratios of asymmetric to symmetric fission fragments to be larger for the $3^{-}$levels than for the $4^{-}$levels.

For ${ }^{239} \mathrm{Pu}$, since the $\mathrm{O}^{+}$resonant states cannot decay 
through saddle point channels that contain mass-asymmetry vibration, some symmetric mass division is expected in these resonances. The $1^{+}$resonant states, as suggested by Griffin ${ }^{(52)}$, are probably a combination state of mass asymmetry and bending vibrations; so symmetric division is expected to be suppressed. Experimental results of Los Alamos Radiochemistry Group (44), Faler and Tromp (45), Regier et al. (46) and Cowan et al. (47) discussed earlier, have been interpreted satisfactorily in terms of the present theory. The experimental results suggest that for ${ }^{239} \mathrm{Pu}$ the $0.297 \mathrm{eV}$ resonant state has spin and parity of $\mathrm{I}^{+}$where symmetric fission is suppressed. In thermal neutron fission, the thermal cross-section is composed of certain amount of contribution from the $0.297 \mathrm{eV}$ resonance plus a larger contribution from the negative energy resonance believed to have spin and parity of $\mathrm{O}^{+}$.

Other types of experimental evidences in support of Bohr's theory include:

(i) the definite variations of fragment kinetic energy from level to level correlated with the spin states of the compound nucleus as reported by Melkonian and Mehta ${ }^{(53)}$ for ${ }^{235} \mathrm{U}$ and ${ }^{239} \mathrm{Pu}$. (ii) the significant variations of the mean number of neutron emitted per fission in a systematic manner among the resonances as observed by Weinstein et al. (54) for ${ }^{233} \mathrm{U},{ }^{235} \mathrm{U}$ and ${ }^{239} \mathrm{Pu}$. The spins for neutron resonances can be directly determined from neutron scattering measurements. Comparisons 
of the direct and indirect spin assignments based on Bohr's theory are displayed in Table 1 and Table 2 for ${ }^{235} \mathrm{U}$ and ${ }^{239} \mathrm{Pu}$ respectively. The general agreement shows definite correlations of some of the post-scission characteristics, such as mass-yield and kinetic energy distribution and the neutron multiplicity with the spins of the compound nucleus at the saddle point. However, the results of Croall and willis ${ }^{(48)}$ on the mass yield variations of the very asymmetric fission fragments $\left({ }^{77}\right.$ As and ${ }^{78}$ As $)$ for thermal and epi-cd neutron fission of ${ }^{239} \mathrm{Pu}$ described earlier, are unable to be accounted for with the present theory. In addition, it is worthwhile noting that popa et al. (63) reported significant fluctuations in the relative yields of asymmetric fission products for thermal and epi-ca neutron induced fission of ${ }^{235} \mathrm{U}$. Wheeler's extension of Bohr's theory also seems unalbe to provide a consistent interpretation. As shown by the works of Regier et al.(46) and Faler and Tromp (45), the spin state effect is much larger for ${ }^{239} \mathrm{Pu}$ than for ${ }^{235} \mathrm{U}$ in the variations of the symmetric yields. It is therefore expected that any effect in the asymmetric fission yields that may be related to the spin states of the compound nucleus at the saddle point should be more drastic for ${ }^{239} \mathrm{Pu}$ than for ${ }^{235} \mathrm{U}$. Another purpose of this work was to investigate carefully such a possibility for the thermal and epi-cd and epi-sm fissions of ${ }^{239} \mathrm{Pu}$. 
TABLE 1

${ }^{235_{U}}$ Resonance Spin (J) Assignments

DIRECT

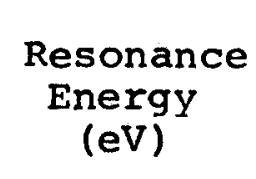

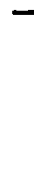
et al. (56)
Asghar et al. (55)

Bowman et al. (57)

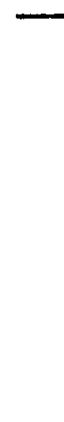

15.40

16.08

16.69

18.07

19.31

21.08

22.39

22.95

23.42

23.65

27.82

30.86

32.07

33.53

34.39

35.26

39.41
4
3
3
3
3
4
4
3

(4)*

3

3

4

3

3

(4)

4

4

4

4

4

3

3

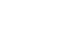
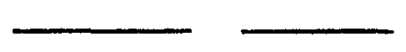

4

3

3

*$$
4
$$

3

3

3

3

3

3

3

3
3

3

\section{INDIRECT}

\begin{tabular}{l} 
Cowan Melkonian Weinstein \\
$\begin{array}{l}\text { et al. \& Mehta } \\
(47)\end{array}$ \\
\hline
\end{tabular}

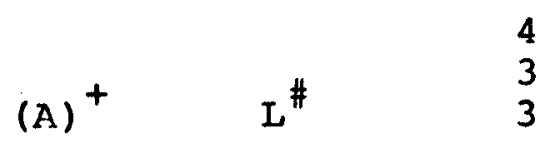

4
3
3

4

A $\mathrm{H}$

A

A

(A)

A

A

A

A $\mathrm{H}$

A

H

$\begin{array}{ll}\text { A } & \text { H } \\ \text { A } & \text { L } \\ \text { S } & \text { L } \\ \text { S } & \text { L } \\ \text { S } & \text { L }\end{array}$

* Uncertainty of assignments.

$+S=$ increased symmetric fission, $A=$ decreased symmetric fission.

\# $I=$ lower average kinetic energy of fragment, $\mathrm{H}=$ higher average kinetic energy of fragment. 
TABLE 2

${ }^{239} \mathrm{Pu}$ Resonance Spin (J) Assignments

DIRECT

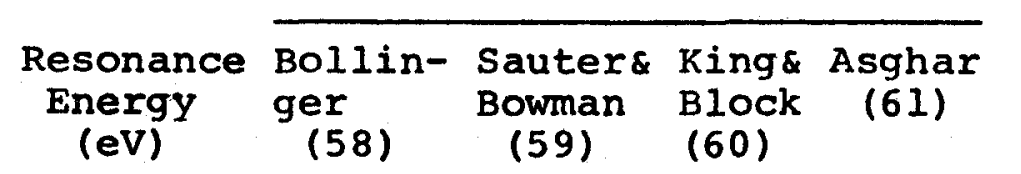

Cowan Melko- Farrell Weinsetal. nian \& (62)

(47) Mehta (53) tein et al. (54)

\begin{tabular}{|c|c|c|c|c|c|c|c|c|}
\hline 0.298 & 1 & & & & & & & 1 \\
\hline 7.85 & 1 & 1 & & & & & & 1 \\
\hline 10.95 & 1 & 1 & & & & & & 1 \\
\hline 11.9 & 1 & $i$ & & & & & & 1 \\
\hline 14.3 & & 1 & & & & & 1 & 1 \\
\hline 14.7 & 0 & 1 & & 1 & & & 1 & 1 \\
\hline 15.5 & 0 & 0 & & & 1 & & 0 & 0 \\
\hline 17.7 & 1 & 1 & & 1 & 1 & & 1 & 1 \\
\hline 22.3 & & 1 & & 1 & 1 & 1 & 1 & 1 \\
\hline 23.9 & & & & & & & 1 & 1 \\
\hline 26.2 & & & & 1 & 1 & (1) & 1 & 1 \\
\hline 32.3 & & 0 & & & $(0) *$ & 0 & 0 & 0 \\
\hline 41.5 & 0 & 1 & 1 & 1 & 1 & $(0)$ & 1 & 1 \\
\hline 44.5 & 1 & 1 & 1 & 1 & 1 & (1) & 1 & 1 \\
\hline 47.6 & & 0 & & 0 & 1 & 1 & 0 & 0 \\
\hline 52.6 & 1 & 1 & 1 & 1 & 1 & 1 & 1 & $(0)$ \\
\hline 62.7 & & & & & & & 0 & 0 \\
\hline 75.0 & & 1 & 1 & 1 & 1 & 1 & 1 & 1 \\
\hline 81.0 & & & & & 0 & & 0 & 0 \\
\hline 90.0 & & & 0 & 1 & & & & 0 \\
\hline
\end{tabular}

* Parentheses indicate uncertain assignment. 
CHAPTER 2

\section{EXPERIMENTAL PROCEDURES}

\section{A. Introduction}

The variations in the mass yields of several symmetric and very asymmetric products in the epi-thermal fission as compared with thermal fission of ${ }^{239} \mathrm{Pu}$ have been studied in this work by means of radiochemical methods. These nuclides included ${ }^{72} \mathrm{zn},{ }^{77} \mathrm{Ge},{ }^{77} \mathrm{As},{ }^{112} \mathrm{Pd},{ }^{115} \mathrm{Cd}$ and ${ }^{166} \mathrm{Ho}$. High yield products from asymmetric fission have also been investigated by nondestructive $G e(L i)$ spectroscopic measurements of the gross fission products (64). Brief information on these nuclides is given below.

The cumulative yield of ${ }^{72} \mathrm{zn}$ in the thermal neutron induced fission of ${ }^{239} \mathrm{Pu}$ is $1.2 \times 10^{-4} \%$ (29). This isotope cannot be produced by reactions other than fission. It has a half-life of $46.5 \mathrm{hr}$ and emits gamma-rays of $144.8 \mathrm{keV}(908)$ and 192.0 keV (10\%) with intensities shown in parentheses. According to Croall and willis (48), the yield of ${ }^{77}$ As is $7.3 \times 10^{-3} \%$ in the thermal fission of ${ }^{239} \mathrm{Pu}$. The branching of the $A=77$ mass chain is quite complicated and is illustrated in Fig. 6. About 978 of $77_{\text {As decays to the ground level of }}{ }^{77} \mathrm{Se}$ while the rest is associated with gamma-rays of $239.3 \mathrm{keV}$ (2.58) and $250.1 \mathrm{keV}(0.28)(66)$. Gamma-ray spectra following the decay of ${ }^{77} \mathrm{Ge}$ show a number of gamma transitions, the most prominent ones 


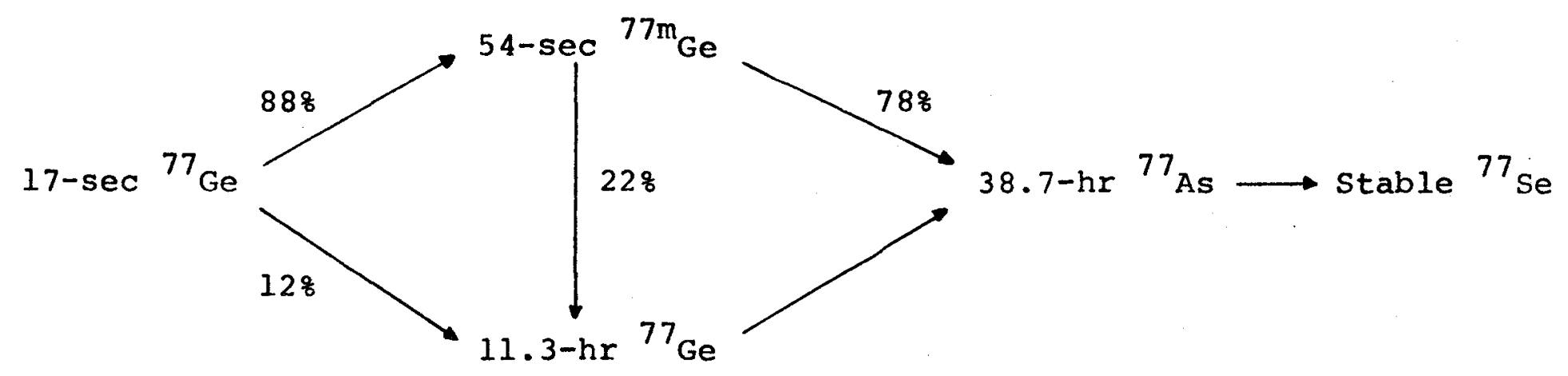

Fig. 6. Branching Scheme of the $A=77$ Mass Chain. 
are $211.0,215.6$ and $264.6 \mathrm{keV}$ with relative intensities of 50 , 48 and 100 respectively $(67)$.

The $21-\mathrm{hr}{ }^{112} \mathrm{Pd}$ decays to the ground state of $3.2-\mathrm{hr}$ ${ }^{112} \mathrm{Ag}$ which in turn emits gamma-rays with energies of $618.8 \mathrm{keV}$ $(100), 606.1 \mathrm{keV}(8), 691.5 \mathrm{keV}(1.1)$ and $694.2 \mathrm{keV}(6.8)(66)$. The yield of ${ }^{112} \mathrm{Pd}$ was reported to be 0.128 in the thermal fission of ${ }^{239} \mathrm{Pu}(29)$.

The yield of another symmetric fission product, ${ }^{115} \mathrm{Cd}$, is $3.8 \times 10^{-2} \%(29)$. The half-life of this isotope is $53.5-\mathrm{hr}$ and decays to ${ }^{115 m}$ n and ${ }^{115}$ In emitting gamma-rays of $492.1 \mathrm{keV}$ (10) and $527.7 \mathrm{keV}(26)$. A gamma-ray of $336.2 \mathrm{keV}$ is associated with the isomeric transition of $115 \mathrm{~m}_{\mathrm{In}}$.

of the heavy nuclides from very asymmetric fission, the $A=166$ mass chain seems to be the only one suitable for this study. The yield of ${ }^{166}$ Dy according to Katcoff (29) is $6.8 \times 10^{-5} 8$. Because of the close similarity of dysprosium and yttrium in chemical properties, radiochemical separation of ${ }^{166}$ Dy from highyield yttrium isotopes and other rare earth fission products presents a major problem. From a consideration of the half-lives and the decay schemes of the 81.5-hr ${ }^{166}$ Dy and the $26.9-\mathrm{hr}$ daughter $\left({ }^{166} \mathrm{HO}\right)$, it was decided to isolate ${ }^{166}$ Ho instead of ${ }^{166}$ Dy, after a state of transient equilibrium has been reached. Approximately 508 of the ${ }^{166}$ HO decays with the emission of an $80.6 \mathrm{keV}$ gamma-ray.

B. ${ }^{239} \mathrm{Pu}$ Sample Preparation and Irradiation

In each experiment, two samples of ${ }^{239} \mathrm{Pu}$ were irradiated 
simultaneously in the core of the McMaster swimming-pool reactor, one with either samarium or cadmium shielding, and the other one without shieldings. The simultaneous irradiations eliminate any necessity for saturation factor correction. The shieldings served to accentuate fissions in the resonance region while minimizing thermal neutron fission. Both types of shielding cut off thermal neutron effectively but the cut off energy is lower for samarium $(\sim 0.2 \mathrm{eV})$ than for cadmium $(\sim 0.5 \mathrm{eV})$. Radiochemical studies using monoenergetic neutrons for the very low yield fission products such as ${ }^{72} \mathrm{zn}$ and ${ }^{166} \mathrm{DY}-{ }^{166}$ Ho would not be feasible because of the low neutron flux available through a monochromator.

The samarium shielding used was a solid cylinder made of commercial cement to which $358 \mathrm{Sm}_{2}{ }_{3}$ by weight had been added and mixed uniformly. The sm-cement cylinder consisted of two parts, the bottom part was cast in an ordinary aluminum can used as the reactor irradiation container. The size of the sm-cement cylinder was $2 \mathrm{~cm}$ in diameter, $4 \mathrm{~cm}$ in height, with a hole at the centre to Which a sealed quartz capillary (2 $\mathrm{mm}$ in diameter, $1 \mathrm{~cm}$ in length) containing the plutonium sample could fit in. The upper part acted like a plug, $2 \mathrm{~cm}$ in diameter and $1 \mathrm{~cm}$ in height. It was calculated that the sample in centre position was being shielded by $1.8 \times 10^{21}$ atoms of $\mathrm{sm} / \mathrm{cm}^{2}$. However, the shielding made in this way is expected to be less effective compared to that made from pure samarium metal as used by Regier etal. (46), because of the perturbation effect of the sm-cement on the neutron flux, and 
"windows" in the Sm-cement as a result of imperfection in packing. Cadmium sheet of $1 \mathrm{~mm}$ in thickness was used to wrap around the sealed quartz capsule which contained the plutonium sample for shielding purposes. The effective shielding was calculated to be $4.6 \times 10^{21}$ atoms of $\mathrm{Cd} / \mathrm{cm}^{2}$.

The samples were PuAl-alloy containing 13.98 plutonium(96.18 ${ }^{239} \mathrm{Pu}$ ). Typically the amount of ${ }^{239} \mathrm{Pu}$ in the unshielded sample was $\sim 0.1 \mathrm{mg}$ and 10 and 20 times more respectively for the samarium and cadmium shielded ones. Before sealing into the quartz tube, the PuAl-alloy was wrapped in $0.001 "$ thick aluminum foil serving as recoil fission-product catcher.

The positions in the reactor core for the shielded and non-shielded samples were chosen in such a way that the total number of fission events in both samples were not very different, usually in the order of $10^{13}$ to $10^{14}$. The thermal neutron fluxes were $2.0 \times 10^{13}$ and $1.4 \times 10^{13} \mathrm{n} / \mathrm{cm}^{2}-\mathrm{sec}$ respectively for the shielded and nonshielded samples.

Cadmium ratios for both manganese and gold of these positions were measured as follows. Cadmium cylinders $1 \mathrm{~cm}$ in diameter and $3 \mathrm{~cm}$ in height with wall thickness of $0.8 \mathrm{~mm}$ were used. The samples of manganese and gold were in solution form $(12 \mathrm{mg} / \mathrm{ml}$ for $\mathrm{Mn}$ and $5.6 \mathrm{mg} / \mathrm{ml}$ for $\mathrm{Au}$ ) so that self-shielding correction can be avoided. They were sealed in small quartz capillaries for irradiations. The cadmium ratios were determined by counting the 
${ }^{56} \mathrm{Mn}(846.9 \mathrm{keV})$ and ${ }^{198} \mathrm{Au}(411.8 \mathrm{keV})$ activities using a $40-\mathrm{cm}^{3} \mathrm{Ge}(\mathrm{L} i)$ detector system, and then comparing the count rates of the non-shielded and the cd-shielded samples after necessary corrections of sample weights and decay factors.

The contribution of fast neutron induced fission in the shielded samples was estimated in one run by irradiating a known amount of metallic $232 \mathrm{Th}$ in similar shielding and in the same position, soon after the completion of the irradiation of the shielded ${ }^{239} \mathrm{Pu}$ sample. Fission product gamma-ray spectra for $232 \mathrm{Th}(n, f)$ were recorded with the Ge(Li) counter and compared with the gross fission product gamma-ray spectrum obtained for the shielded ${ }^{239} \mathrm{Pu}$ sample under similar conditions. Since ${ }^{232}$ gh fission can be caused by fast neutrons only, the contribution of fission by fast neutrons in the ${ }^{239} \mathrm{Pu}$ sample can be estimated from the comparison.

In the studies of ${ }^{72} \mathrm{zn},{ }^{112} \mathrm{Pd}$ and ${ }^{115} \mathrm{Cd}$, only thermal. and epi-Sm fission measurements were made. The samples prepared in the way as described above, were irradiated for six hours in one run and four hours in the other. Both were cooled for $\sim 24$ hours before carrying out the radiochemistry. The total number of fission events in the samples were $\sim 10^{13}$.

For ${ }^{77} \mathrm{Ge}$, both thermal to epi-Cd and thermal to epi-sm fissions were studied. The samples were irradiated for 2 hours in the case for epi-Sm and 4 hours for epi-cd experiments; both were cooled for $\sim 12$ hours. A total of $\sim 10^{13}$ fissions occured in each 
sample.

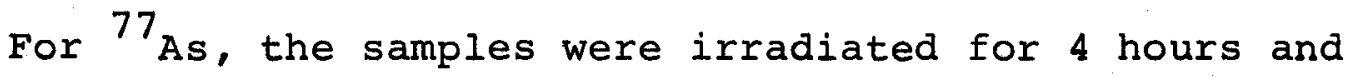
cooled for 36 hours. Only thermal and epi-cd fissions were studied.

Since the yield of the $A=166$ chain is very low, the samples were required to be irradiated for 20 hours to obtain $2 \times 10^{14}$ fission events in each. A cooling period of 100 hours was necessary because of the high level of radiation of the 47-hr ${ }^{153} \mathrm{Sm}$ produced in the samarium shielding during the irradiation.

\section{Ge(Ii) Gamma-ray Spectroscopy}

High resolution $\mathrm{Ge}(\mathrm{I} i)$ detectors developed since the midnineteen sixties have not only been very useful in studying the fission energetics but also relieved some unnecessary and tedious radiochemistry in fission mass-yield and fragment range measurements (64), (68). For a number of the high-yield fission products, prominent gamma rays of specific isotopes have been found to be well resolved in the gamma-ray spectrum recorded with an ordinary $\mathrm{Ge}(\mathrm{Li})$ counter (64), without any radiochemical treatment. Hence, relative fields of the high-yield fission products of any fission can be directly obtained by comparing with the Ge(Ii) spectrum of the gross fission products, usually of the thermal neutron fission of ${ }^{235} \mathrm{U}$, for which the mass-yield distribution is well-known. This technique has been employed in this work to study fluctuations in the relative yields of the asymmetric products in the epi-cd and epi-Sm fissions of ${ }^{239} \mathrm{Pu}$ as compared with the thermal fission. 
After the irradiation and an appropriate period of cooling, the quartz-sealed samples were washed with aqua regia and distilled water. The quartz capsules were then crushed open and the samples including the aluminum catcher foil were transferred to small glass vials. The epi-thermal and thermal neutron-irradiated samples were each counted twice in identical geometry at a distance of about $1 \mathrm{~m}$. away from the $\mathrm{Ge}(\mathrm{L} i)$ detector. The detecting system includes a $12-\mathrm{cm}^{3}$ co-axial $\mathrm{Ge}(\mathbb{I} 1)$ detector and a $40-\mathrm{cm}^{3}$ Nuclear Diodes circular closed-end co-axial Ge(Li) detector, coupled through an Ortec FET preamplifier and an ORTEC-435 amplifier to a Nuclear Data, Series 2200, 1024 channel analyser. The system resolution for both detectorswas $4.5 \mathrm{keV}$ FWHM at $1.33 \mathrm{MeV}$. Each counting was set at $1000 \mathrm{sec}$ live-time and the timing was controlled by feeding pulses from a pulser during the measurement. No discrepencies were found for the live timer and the results from the pulser. The dead time of the analyzer never exceeded $10 \%$.

\section{Radiochemistry \\ 1. Carrier solution preparations}

In all the radiochemical procedures to be described, a known amount of the carrier elements of the isotopes of interest were added when dissolving the sample. The percentage of recovery was determined after completion of the radiochemical treatment. Exchange between carrier ions and the radioisotopes of the same element was ensured, and the percentage chemical yield of the 
element should represent the percentage of recovery of the radioactive isotope.

For the experiments on ${ }^{72} \mathrm{zn},{ }^{112} \mathrm{Pd}$ and ${ }^{115} \mathrm{Cd}$, a mixed carrier solution of zinc, palladium and cadmium was prepared by dissolving the metals (analytical grade) of these elements in dilute $\mathrm{HCl}$ and a little of $\mathrm{HNO}_{3}$. The concentrations of the carriers were $7.96 \mathrm{mg}$ for $\mathrm{Zn}, 7.77 \mathrm{mg}$ for $\mathrm{Pd}$ and $15.75 \mathrm{mg}$ for Cd in a gramme of carrier solution.

The germanium carrier solution $\sim 10 \mathrm{mg} / \mathrm{ml}$ was prepared by dissolving $\sim 0.5 \mathrm{gm}$ germanium metal (99.98) in $30 \mathrm{ml}$ of $38 \mathrm{H}_{2} \mathrm{O}_{2}$ and dilute $\mathrm{HCl}$. This metal dissolves very slowly, and the residue was filtered and the solution made up to $50 \mathrm{ml}$.

A carrier solution of As (III) of $\sim 10 \mathrm{mg} / \mathrm{mI}$ was prepared in the following way. Approximately 1.3gm of dried analytical grade $\mathrm{As}_{2} \mathrm{O}_{3}$ (99.9\%) was dissolved with $20 \mathrm{ml}$ of $2 \mathrm{M} \mathrm{NaOH}$, and $20 \mathrm{ml}$ distilled water was added. This was neutralized with $20 \mathrm{ml} 2 \mathrm{M} \mathrm{HCl}$ and diluted to $100 \mathrm{ml}$.

The carrier solution of holmium was prepared by dissolving $\sim 0.6 \mathrm{gm}$ holmium metal in dilute $\mathrm{HCl}$ and diluted to $50 \mathrm{ml}$.

Since these experiments measured the mass-yield ratios of two samples (thermal and epi-thermal), one only needs to know the relative chemical yields rather than the absolute yields. Therefore, the knowledge of the exact concentration of the carrier solution is of no importance. 
2. Sample dissolution and the removal of ${ }^{239} \mathrm{Pu}$

The ${ }^{239} \mathrm{Pu}$ is a highly hazardous isotope because of its $4.5 \mathrm{Mev} \alpha$-activity $\left(t_{1 / 2}=20,000\right.$ years $)$. Hence, it is desirable to remove this element completely from the fission products as early as possible before any further radiochemical treatments of the samples.

Anion exchange chromatography has been very frequently used in removing plutonium from the gross fission products. Table 3 shows the behavior of plutonium in different oxidation states in HCl solution on a strong base anion exchange resin in the chloride form. Plutonium (IV) in nitric acid solution is also adsorbed on strong base anion exchange resin in the nitrate form (70). The distribution coefficients of $\mathrm{Pu}($ IV) as a function of the concentration on $\mathrm{HNO}_{3}$ is shown in Fig. 7 .

It is essential to have the right oxidation states for adsorption or elution of plutonium from an anion exchange column. The +III and +IV states are the stable and most common forms of plutonium. Oxidizing agents such as $\mathrm{HNO}_{3}, \mathrm{NaNO}_{2}\left(\mathrm{NH}_{4} \mathrm{NO}_{2}\right), \mathrm{FeCl}_{3}$ etc. are able to keep plutonium in the IV state and prevent its reduction. On the other hand, in order to prevent the uptake of plutonium or to achieve rapid elution one can add $\mathrm{HI}, \mathrm{NH}_{4} \mathrm{I}, \mathrm{SO}_{2}$ or ascorbic acid(71).

In the experiments for ${ }^{72} \mathrm{zn},{ }^{112} \mathrm{Pd}$ and ${ }^{115} \mathrm{Cd}$, the samples after the Ge(Li) gross fission product gamma-ray countings were 
TABLE 3

Distribution Coefficients of Plutonium on

strong Base Anion Exchange Resin in $\mathrm{HCl}^{(69)}$

$\mathrm{HCl}$

Concentration

1

(N)

$\mathrm{Pu}(\mathrm{III})$

$\mathrm{Pu}(\mathrm{IV})$

$\mathrm{Pu}(\mathrm{VI})$

\section{no adsorption}

$1<1<1 \quad 30>10^{3}>10^{3}>10^{3}$ strongly adsorbed from $\mathrm{HCl}>6 \mathrm{~N}$ 


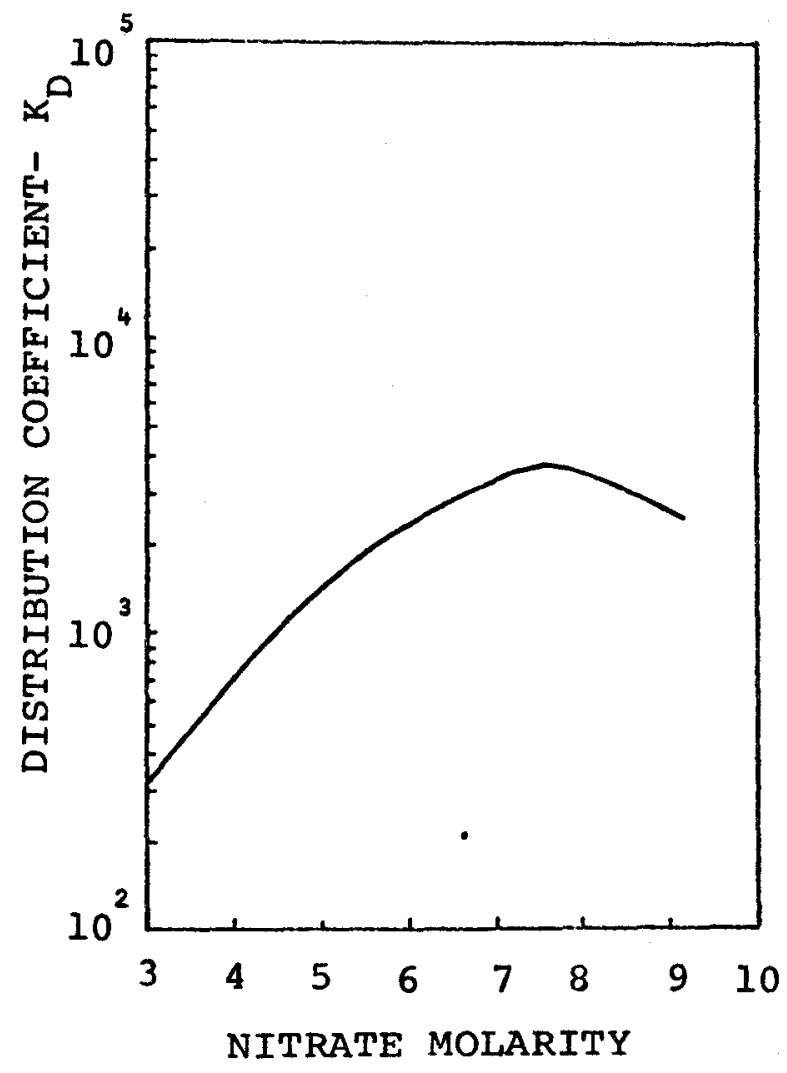

Fig. 7. Distribution coefficient of Pu(IV) on Dowex-1X4 (50-100 mesh) from nitric acid (70). 
dissolved in $2 \mathrm{ml}$ of concentrated $\mathrm{HCl}$ and few drops of concentrated $\mathrm{HNO}_{3}$ in the presence of $1.5 \mathrm{gm}$ of mixed carrier containing $\sim 12 \mathrm{mg} \mathrm{zn}^{2+}$, $12 \mathrm{mg} \mathrm{Pa}^{2+}$ and $20 \mathrm{mg} \mathrm{Cd}^{2+}$. The sample was diluted to $2 \mathrm{M}$ in $\mathrm{HCl}$ and passed through an anion exchange column of Dowex AGlX10, 200-400 mesh, $0.6 \mathrm{~cm} \times 6 \mathrm{~cm}$ in size and preconditioned with $2 \mathrm{M} \mathrm{HCl}$. All three elements were retained on the column while ${ }^{239} \mathrm{Pu}$ in either the +III or the +IV state simply passed through (69) and was completely removed by washing the column with $\sim 20 \mathrm{ml} 2 \mathrm{M} \mathrm{HCl}$.

In the experiments for ${ }^{77} \mathrm{Ge}$, preremoval of ${ }^{239} \mathrm{Pu}$ was not required. The samples were put into $250 \mathrm{ml}$ distillation flasks with associated closed-distillation systems and dissolved by adding $1.00 \mathrm{ml}$ of Ge-carrier solution and loml of $4 \mathrm{M} \mathrm{HCl}$ saturated with $\mathrm{Cl}_{2}$, and then adjusted to $8 \mathrm{M}$ in $\mathrm{HCl}$. Germanium was distilled over in a stream of $\mathrm{Cl}_{2}$ while ${ }^{239} \mathrm{Pu}$ was kept behind in the distillation flask. For ${ }^{77}$ As, a similar distillation procedure was used to leave the ${ }^{239} \mathrm{Pu}$ behind.

For $\quad{ }^{166} \mathrm{HO}$, the samples were dissolved in dilute $\mathrm{HCl}$ and in the presence of $\sim 12 \mathrm{mg} \mathrm{Ho}{ }^{3+}$ carrier and a few arops of concentrated $\mathrm{HNO}_{3}$. The solution was then brought to dryness, taken

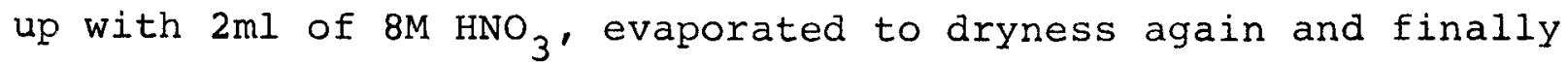
taken up with $2 \mathrm{ml} 8 \mathrm{M} \mathrm{HNO}_{3}$. These steps assured that the plutonium would be in the IV state and in the nitrate form. This solution was then passed through a Dowex AGlX10, 100-200 mesh, $0.6 \mathrm{~cm} \times 6 \mathrm{~cm}$ anion exchange column which had been preconditioned with sufficient $8 \mathrm{M} \mathrm{HNO}_{3}$. The $\mathrm{Pu}(\mathrm{IV})$ was very strongly adsorbed on the column while 
holmium went through.

3. Separation and purification of ${ }^{72} \mathrm{zn}, 112 \mathrm{Pd}$ and ${ }^{115} \mathrm{Cd}$ The group of ${ }^{72} \mathrm{zn},{ }^{112} \mathrm{pd}$ and ${ }^{115} \mathrm{Cd}$ with their carriers were adsorbed on the Dowex $1 \times 10$ anion exchange column with ${ }^{239} \mathrm{Pu}$ removed. Zinc and cadmium were then eluted off with $25 \mathrm{ml}$ of distilled water. After scavenging twice with ferric hydroxide and once with palladium sulfide from a $2 \mathrm{M} \mathrm{HCl}$ solution, the two elements were precipitated simultanously as sulfides. They were dissolved in $2 \mathrm{M} \mathrm{HCl}$ and adsorbed on a column of Dowex AG2X8, 100-200 mesh, $0.6 \mathrm{~cm} \times 16 \mathrm{~cm}$ in size. After washing with $2 \mathrm{M} \mathrm{HCl,} \mathrm{zinc}$ was stripped of with $20 \mathrm{ml}$ of $0.05 \mathrm{M} \mathrm{HCl}$. The eluant was scavenged once more by ferric hydroxide precipitation. Finally, $0.5 \mathrm{gm}$ $\mathrm{NH}_{4} \mathrm{Cl}$ was added to the zinc filtrate of $\sim 20 \mathrm{ml}$, the $\mathrm{pH}$ adjusted to $\sim 6$ using methyl red and bromthymolblue mixed indicator. The zinc was then precipitated as $\mathrm{ZnNH}_{4} \mathrm{PO}_{4}$ from the hot solution with $3 \mathrm{ml}$ of $10 \% \mathrm{NH}_{4} \mathrm{HPO}_{4}$ solution. The precipitate after digestion for $\sim 20 \mathrm{~min}$ on a hot plate, was filtered with a 20ml-chimney and sintered-glass filtering system onto a $3 \mathrm{~cm}-g$ lass fibre filter pad. The precipitate was dried in the oven at $\sim 110^{\circ} \mathrm{C}$ for half an hour and weighed for chemical yield determination.

The cadmium remaining on the Dowex AG2X8 column was eluted with $60 \mathrm{ml}$ of distilled water and then prepared for weighing and counting as $\mathrm{CdNH}_{4} \mathrm{PO}_{4} \cdot \mathrm{H}_{2} \mathrm{O}$ in a similar way as for $\mathrm{zinc}$.

Palladium was eluted from the first anion-exchange column with $1 \mathrm{M} \mathrm{NH}{ }_{4} \mathrm{OH}$ after the elution of $\mathbb{Z n}$ and $\mathrm{Cd}$. It was 
further purified by ferric hydroxide scavenging, sulfide precipitation, dissolution in nitric acid and finally scavenged with silver chloride precipitation. The filtrate of palladium was then adjusted to $0.4 \mathrm{M}$ in $\mathrm{HCl}$ and $\sim 5 \mathrm{ml}$ of 108 dimethylglyoximate solution was added. The precipitate of palladium dimethylglyoximate was filtered onto the glass fibre filter pad, washed with $50 \%$ ethanol, dried at $\therefore 110^{\circ} \mathrm{C}$ for $\sim 20 \mathrm{~min}$ and finally weighed as PaDMG. The chemical yields of $\mathrm{zn}, \mathrm{Cd}$ and $\mathrm{Pd}$ were approximately 80,70 and 908 .

The zinc samples were counted several times for $50 \mathrm{~min}$ each using a $7.5 \mathrm{cmX} 7.5 \mathrm{~cm} \mathrm{NaI(TI)} \mathrm{detector} \mathrm{in} \mathrm{connection} \mathrm{with} \mathrm{a}$ Victorean preamplifier and DD2-amplifier and a Nuclear Data 256 channel analyser. The cadmium and palladium samples were counted using the $12 \mathrm{~cm}^{3} \mathrm{Ge}(\mathrm{Li})$ unit described before. The cadmium samples were measured soon after their preparation and also after an appropriate growing-in period for its daughter, $115 \mathrm{~m}_{\mathrm{In}}$. For palladium samples $(112 \mathrm{Pd})$, countings were carried out via its daughter silver isotope $\left({ }^{112} \mathrm{Ag}\right)$ after a growing-in time of about 20 hours. These radiochemical procedures were based on references (72), (73) and (74).

4. Separation of ${ }^{77} \mathrm{Ge}$

Simple radiochemistry for the separation of $77^{\mathrm{Ge}}$ was possible with a fairly high decontamination factor by specific distillation of $\mathrm{GeCl}_{4}(75)$. The samples were dissolved in the way as described in section $(2-c-2)$ in a closed distillation system. 
The solution was $\sim 20 \mathrm{ml}$ in volume and $8 \mathrm{M} \mathrm{HCl}$. Germanium was distilled off in a stream of $\mathrm{Cl}_{2}$ gas at temperature kept below $110^{\circ} \mathrm{C}$. Five $\mathrm{ml}$ of the distillate was collected in $5 \mathrm{ml}$ of distilled water cooled in an ice bath. Chlorine in the distillate was expelled by passing air through it, and then the solution was adjusted to $6 \mathrm{M}$ in $\mathrm{HCl}$ and cooled in ice. Germanium sulfide was precipitated in the presence of a few $\mathrm{mg}$ of $\mathrm{KI}$, centrifuged and washed with $6 \mathrm{M} \mathrm{H}_{2} \mathrm{SO}_{4}$ presaturated with $\mathrm{H}_{2} \mathrm{~S}$. The precipitate was filtered onto a glass fibre filter pad in the usual way, dried at $110^{\circ} \mathrm{C}$ and weighed as $\mathrm{GeS}_{2}$. The chemical yield ratio was further checked by an activation method after all ${ }^{77} \mathrm{Ge}$ had virtually decayed. The chemical yields were about 958 .

The samples were counted with the $40 \mathrm{~cm}^{3} \mathrm{Ge}(\mathrm{Li})$ detector system. The spectra recorded (to be shown in the next section) revealed the presence of ${ }^{132} \mathrm{Te},{ }^{132} \mathrm{I}$ and ${ }^{135} \mathrm{Xe}$, the latter had grown in from ${ }^{135} \mathrm{I}$. However the decontamination was sufficient for ${ }^{77} \mathrm{Ge}$ activity determination using the prominent $254 \mathrm{keV}$ gamma-ray and the 211-215 keV doublet. The energies of the gamma-rays and the half-life calibrations both confirmed that they were free of interference from other gamma transitions.

Since ${ }^{77}$ Ge may also be produced by stable ${ }^{76} \mathrm{Ge}$ through ${ }^{76} \mathrm{Ge}(n, \gamma)$, the aluminum foil used for wrapping and as a recoil catcher for the samples were also investigated for possible germanium content. As a result, no detectable quantity of germanium was found. 
5. Separation and purification of $77^{\mathrm{AS}}$

There are two common methods of isolating arsenic activities from fission products (75), namely the solvent extraction of As(III) as iodide with $\mathrm{CHCl}_{3}$, and the distillation of As(III) from a hydrochloric acid solution. Since the yield of arsenic activities are relatively low, the solvent extraction or the distillation procedure usually has to be carried out repeatedly to obtain a radiochemically pure sample. Not only is the procedure tedius but it also frequently ends up with low chemical yields.

In the present work, it was found in preliminary experiments that only one cycle of the distillation and further purification with an anion exchange column and sulfide precipitation would give a reasonably clean arsenic sample of fairly high yield.

The plutonium samples were dissolved in $50 \mathrm{ml}$ distillation flasks by adding $2 \mathrm{ml}$ of $\mathrm{As}^{3+}$ carrier solution $\left(\sim 13 \mathrm{mg} \mathrm{As}^{3+}\right)$ and a few mg each germanium carrier and tellurium hold-back carrier, and $6 \mathrm{ml}$ of $9 \mathrm{M} \mathrm{HCl}$, in the presence of $\mathrm{Cl}_{2}$ gas. The ${ }^{77} \mathrm{Ge}-{ }^{77} \mathrm{As}$ separation was properly achieved by distilling ${ }^{77}$ Ge off both samples side by side within $20 \mathrm{~min}$ in a stream of $\mathrm{Cl}_{2}$ gas. The volume of distillate collected was $\sim 4 \mathrm{ml}$. Since ${ }^{77}$ As and its carrier would be in the $+V$ state under such conditions, it remained in the solution (75).

The $\mathrm{Cl}_{2}$ in solution was then expelled by passing nitrogen through. A suspension of $0.3 \mathrm{ml}$ hydrazine hydrate and $13 \mathrm{ml}$ of concentrated $\mathrm{HCl}$ were added into the distillation flask together with $0.25 \mathrm{gm}$ of boric acid dissolved in $7 \mathrm{ml}$ of conc HCl. Twenty milliliters of the solution was then distilled off using nitrogen 
as a carrier gas and the distillate was collected in $10 \mathrm{ml}$ of ice-cooled water. After adding another $20 \mathrm{ml}$ of conc.HCl into the distillation flask a further $20 \mathrm{ml}$ of distillate was collected. The arsenic in the +III state was distilled over.

The distillate was then diluted to $4 \mathrm{M}$ in $\mathrm{HCl}$ and passed through an anion exchange column of Dowex AGłxl0, 100-200 mesh, $1.0 \mathrm{~cm} \times 10 \mathrm{~cm}$ and preconditioned with $4 \mathrm{M} \mathrm{HCl}$. Contaminants carried over in the distillate such as ${ }^{132} \mathrm{Te}$ and the iodine isotopes were efficiently adsorbed on the column while $\mathrm{As}^{3+}$ passed through.

Finally the arsenic in the filtrate was precipitated with $\mathrm{H}_{2} \mathrm{~S}$. The $\mathrm{As}_{2} \mathrm{~S}_{3}$ was filtered, dried and weighed in a similar way as described for zinc. Chemical yield ratios of the thermal to epi-cd samples were obtained and further checked by the reactivation method (by ${ }^{75}$ As $(n, \gamma){ }^{76}$ As) after completion of radiocounting. The chemical yield was $\sim 80 \%$.

The samples were counted with the $40 \mathrm{~cm}^{3} \mathrm{Ge}(\mathrm{Li})$ system. The gamma-ray spectra, to be presented in the next chapter, showed that a clean ${ }^{77}$ As was obtained.

It is interesting to note that if no tellurium(IV) was added to the solution in the distillation flask as a hold-back carrier, the minor amount of ${ }^{132}$ Te carried through was impossible to remove by the anion exchanger.

6. Separation and purification of ${ }^{166}$ HO

Rare earth elements have been frequently separated as a group as fluorides and then separated from each other on cation 
exchange columns using $\alpha$-hydroxy-isobutyric acid $(\alpha-H I B)$ as an eluant $(77)$. A reproducible pattern of elution in the order that rare earths with higher atomic numbers are eluted in the earlier fractions than the lighter ones can always be obtained with these elements present in the +III state. The relative distribution coefficients are shown in Table 4. Note that the position of yttrium, chemically behaving like a rare earth element, nearly completely overlaps with dysprosium and is close to holmium.

Since the differences in the distribution coefficients of adjacent heavier rare earths are very small in the cation exchange system using $\alpha-H I B$ as eluant, good separation could only be obtained if the $\mathrm{pH}$ and concentration of the eluting agent are carefully adjusted. Figure 8 clearly shows the effect of the pH on the elution positions of the elements. The choice of resin of different cross-linkages is also of relevance. While resins of higher cross-linkages give good separation for certain rare earths at increased temperature, similar separation can be achieved using resin with lower cross-linkage at room temperature. (79) The latter is often preferred because difficulties arise at higher temperatures.

At an early stage of this work an attempt was made to obtain a complete separation of dysprosium from yttrium, specifically to obtain a clean ${ }^{166}$ Dy activity (with carrier) out of $\sim 10^{6}$ times as much carrier-free yttriumactivities in the fission products. This was often possible using an anion exchange resin in the 
TABLE 4

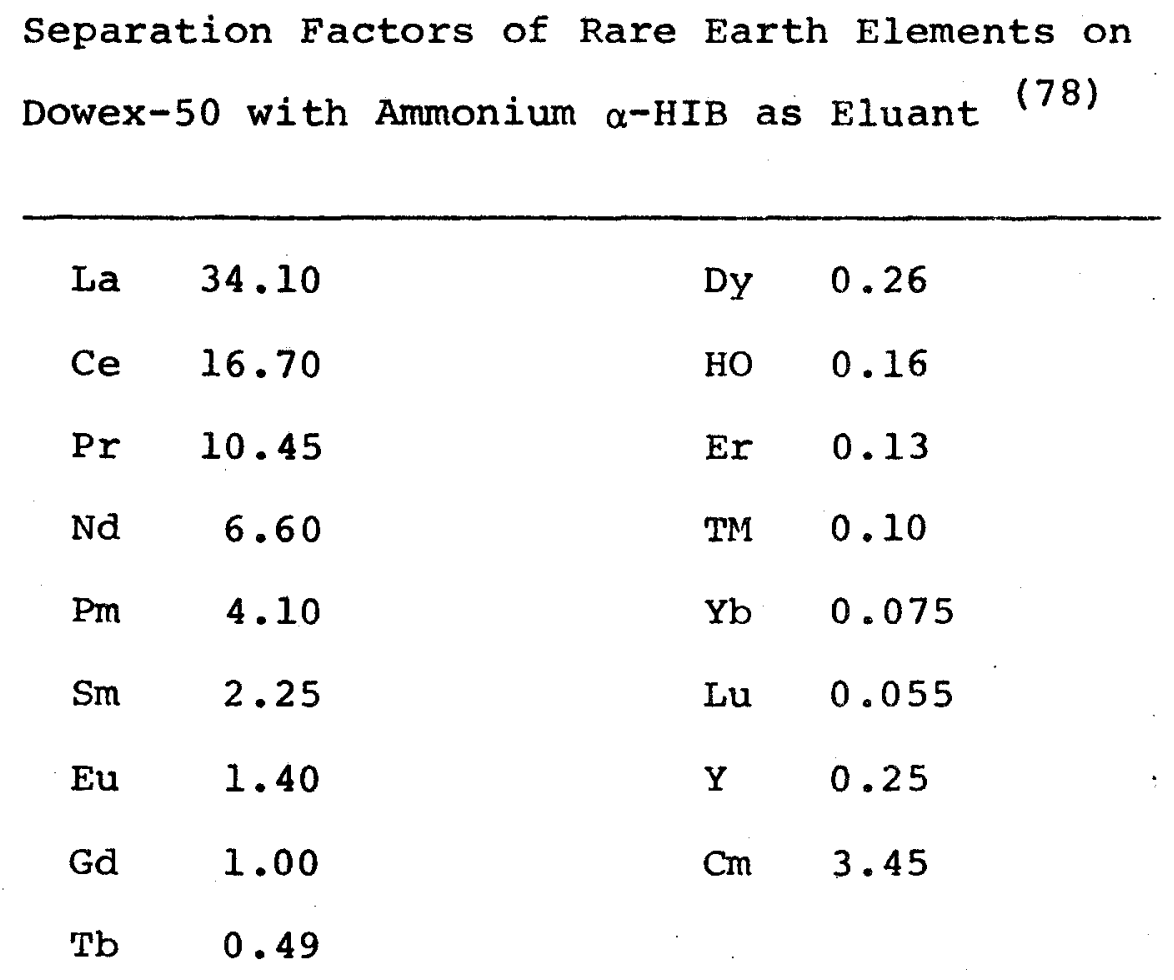




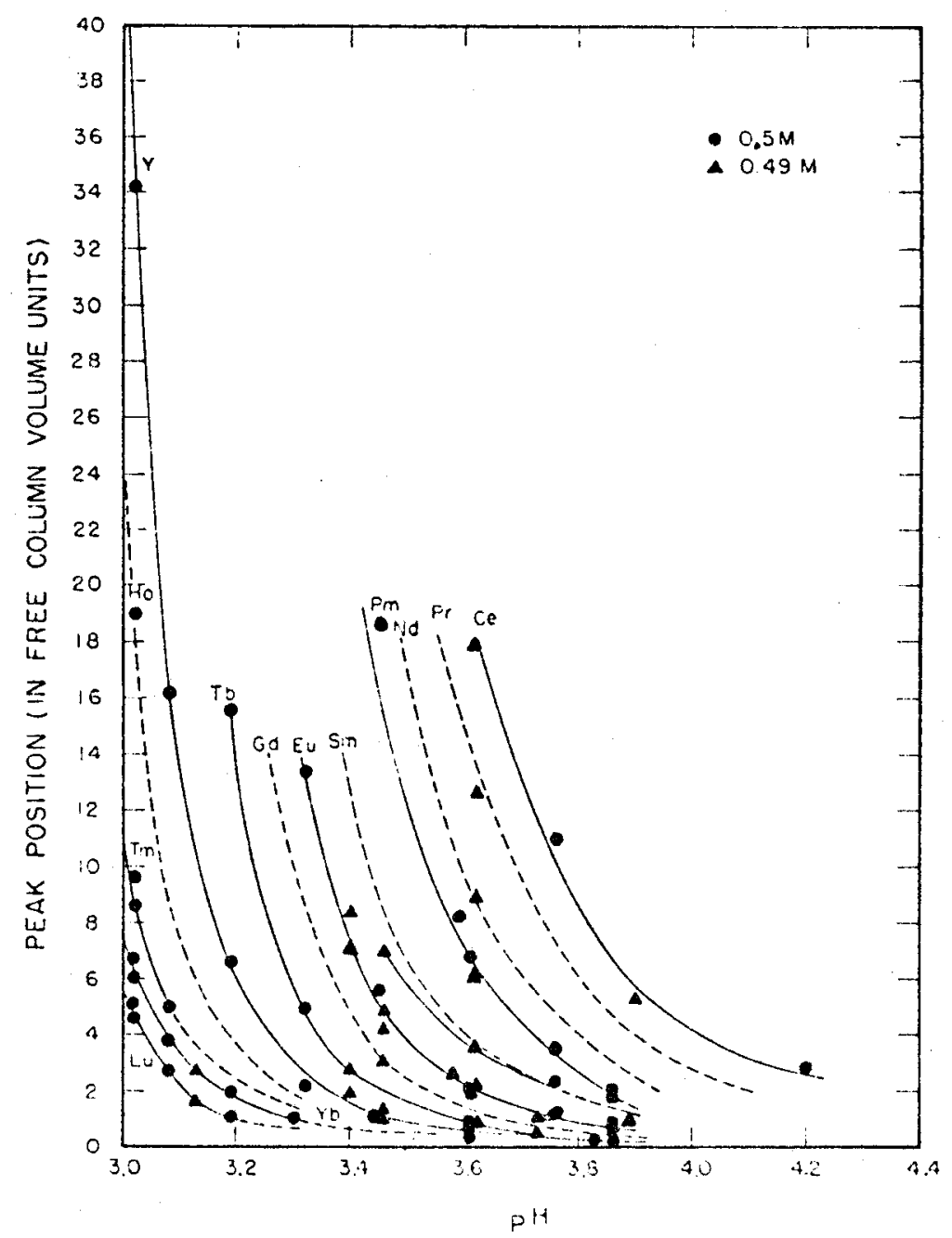

Fig. 8. Elution peak position as a function of $\mathrm{pH}$ for trace lanthanides from Dowex-50x4 with $0.5 \mathrm{M}$ ammonium $\alpha$-HIB (78). 
ethylenediaminetraacetate (EDTA) form and eluting with dilute disodium-EDTA solution. Figure 9 shows the distribution coefficients of the lanthanides on the anion exchange resin in the EDTA form. Successful separation of ${ }^{165}$ Dy from yttrium and other rare earths has also been reported by Alimarin et al. (81). The resin we used was Dowex AGlX10, 200-400 mesh, $0.6 \mathrm{~cm} \times 25 \mathrm{~cm}$ and preconditioned with $1 \mathrm{M}$ ammonium acetate, $\mathrm{H}_{2} \mathrm{O}$ and then with $0.1 \mathrm{M} \mathrm{Na} \mathrm{Na}_{2}$-EDTA solution until free of chloride ion. Dysprosium activity with lomg carrier which has been separated as a group of rare earths was precipitated as hydroxide, dissolved in $2 \mathrm{MHCl}$, evaporated to dryness and finally taken up with $1 \mathrm{ml}$ of $0.005 \mathrm{M} \mathrm{Na}_{2}$-EDTA solution. This was transferred to the column and then eluted with $0.01 \mathrm{M}$ $\mathrm{Na}_{2}$-EDTA solution at a flow rate of 1 drop/min. Yttrium was eluted before dysprosium. Radiochemically pure dysprosium free of yttrium activity was often obtained.

This attempt to isolate ${ }^{166}$ Dy was dropped because of the following difficulties: First, radiochemical purity was not always reproducible. Second, the complete removal of EDTA from the dysprosium solution is essential so that the chemical yield determination would not be affected. However, this always ends up with additional loss of chemical yield. Third, since the separation scheme took about 24 hours, the equilibrium of ${ }^{166}{ }_{\mathrm{DY}}{ }^{166_{\mathrm{HO}}}$ might be affected unpredictably; and the energy of the gamma-ray of ${ }^{166}$ Dy $(82.5 \mathrm{keV})$ is very close to that of ${ }^{166}$ Ho $(80.6 \mathrm{keV})$, thus counting with NaI(TI) detector may give erroneous result. The procedure developed in this study for the separation 


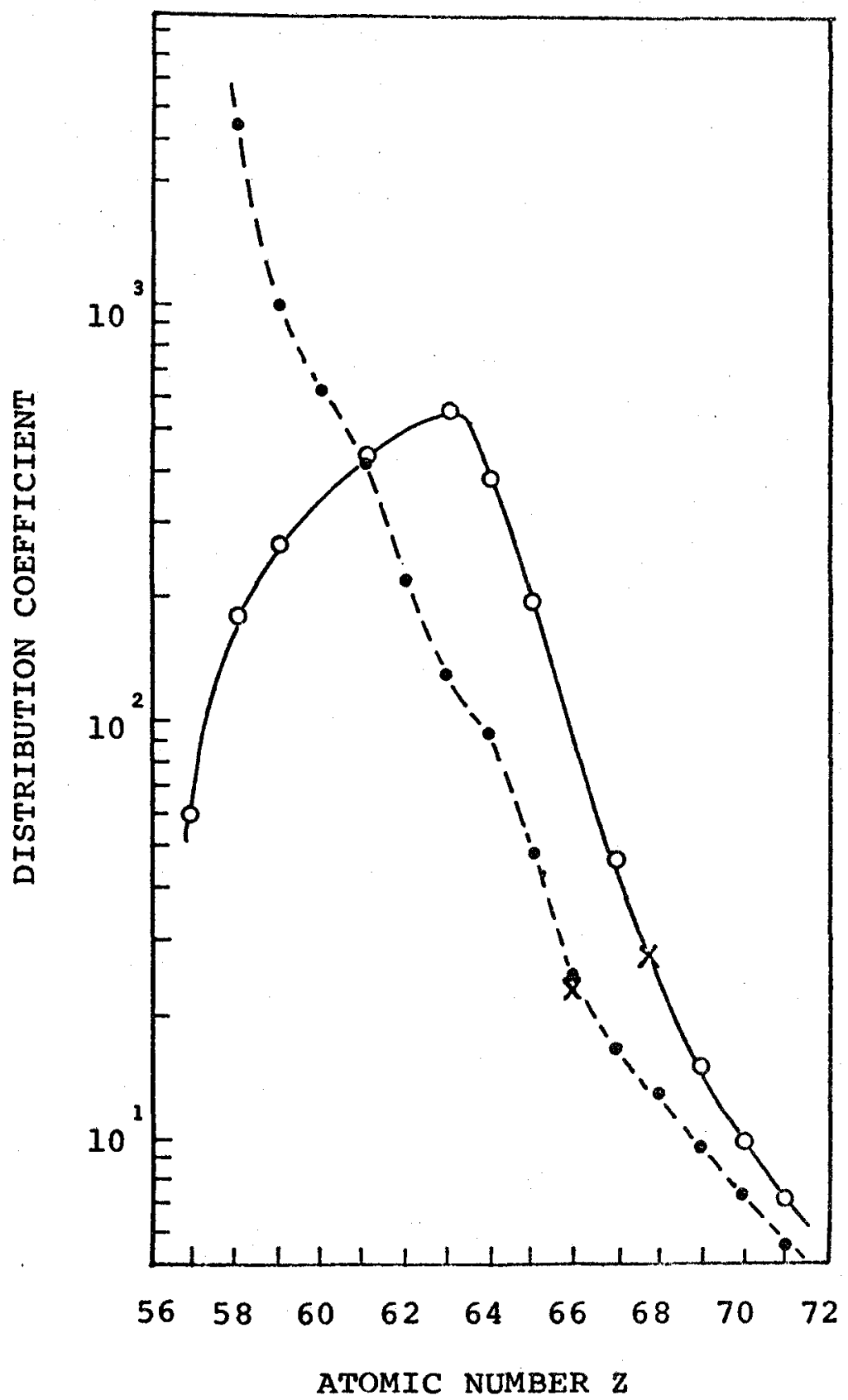

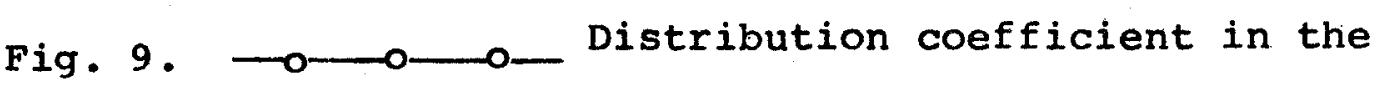

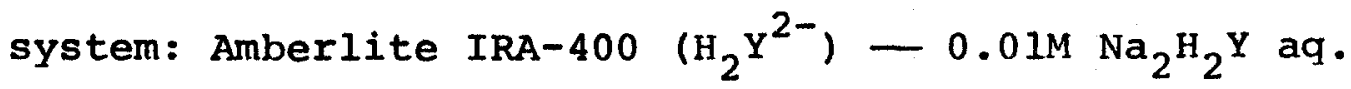
$(Y=$ EDTA $)$. - - - Relative distribution coefficients (Gd taken as standard) in the system: Dowex-50X12 - $\alpha$-HIB at $87^{\circ} \mathrm{C}(80) . x=y$ ttrium. 
of holmium from the rest of the rare earths and yttrium isotopes was based on reference (82). The cation exchange resin of Dowex$50 \times 4$, minus 400 mesh was treated with $6 \mathrm{M} \mathrm{HCl}, 1 \mathrm{M} \mathrm{NH} 4 \mathrm{SCN}, 6 \mathrm{M} \mathrm{HCl}$, $\mathrm{H}_{2} \mathrm{O}, 1 \mathrm{M} \mathrm{NH} 4 \mathrm{OH}$ and $\mathrm{H}_{2} \mathrm{O}$, in the respective order. The size of the column was $1 \mathrm{~cm} \times 36 \mathrm{~cm}$, and $0.5 \mathrm{M} \alpha-\mathrm{HIB}$ of $\mathrm{pH} 3.16$ was used as eluting agent. The sample which contained the rare earth activities and $\sim 13 \mathrm{mg}$ of $\mathrm{HO}^{3+}$ carrier was usually taken up with minimum amount $(\theta .3 \mathrm{ml})$ of the same resin and then transferred onto the column. It was then washed with $2 \mathrm{ml}$ of the eluting agent and then eluted under controlled pressure to adjust the flow rate to $\sim 7 \mathrm{ml} / \mathrm{hour}$. Fig. 10 shows an elution curve obtained under the described condition in

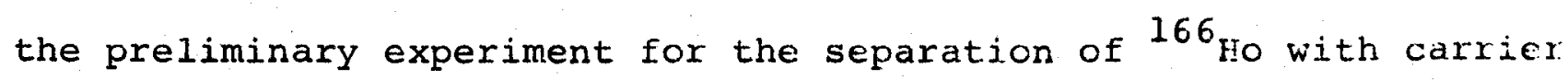
from ${ }^{165}$ Dy tracer. Fig. 11 shows a similar elution curve for the separation of stable holmium and carrier free ${ }^{90} \mathrm{Y}$. Since complete separation was not obtained, it was necessary to discard minor portions in the overlapping region.

The detailed procedure for the isolation of ${ }^{166_{\text {Ho }}}$ from the gross fission products is given below.

The solution of the sample from which ${ }^{239} \mathrm{Pu}$ had been removed by an anion exchanger in the nitrate form was in $8 \mathrm{M} \mathrm{HNO}_{3}$ and contained the holmium carrier and the fission products. The $\mathrm{Ho}(\mathrm{OH})_{3}$ was precipitated with $\mathrm{NH}_{4} \mathrm{OH}$ and then dissolved in $2 \mathrm{M} \mathrm{HCl}$ and precipitated as $\mathrm{HoF}_{3}$ with $38 \% \mathrm{HF}$. The fluoride was digested on a hot bath, centrifuged and washed with $0.1 \mathrm{M} \mathrm{HF}$, and then decomposed by adding $3 \mathrm{ml}$ saturated hydroboric acid solution and $2 \mathrm{ml}$ conc $\mathrm{HNO}_{3}$. The $\mathrm{Ho}(\mathrm{OH})_{3}$ was precipitated again from the diluted solution, dissolved in conc $\mathrm{HCl}$ and passed through an anion exchange 


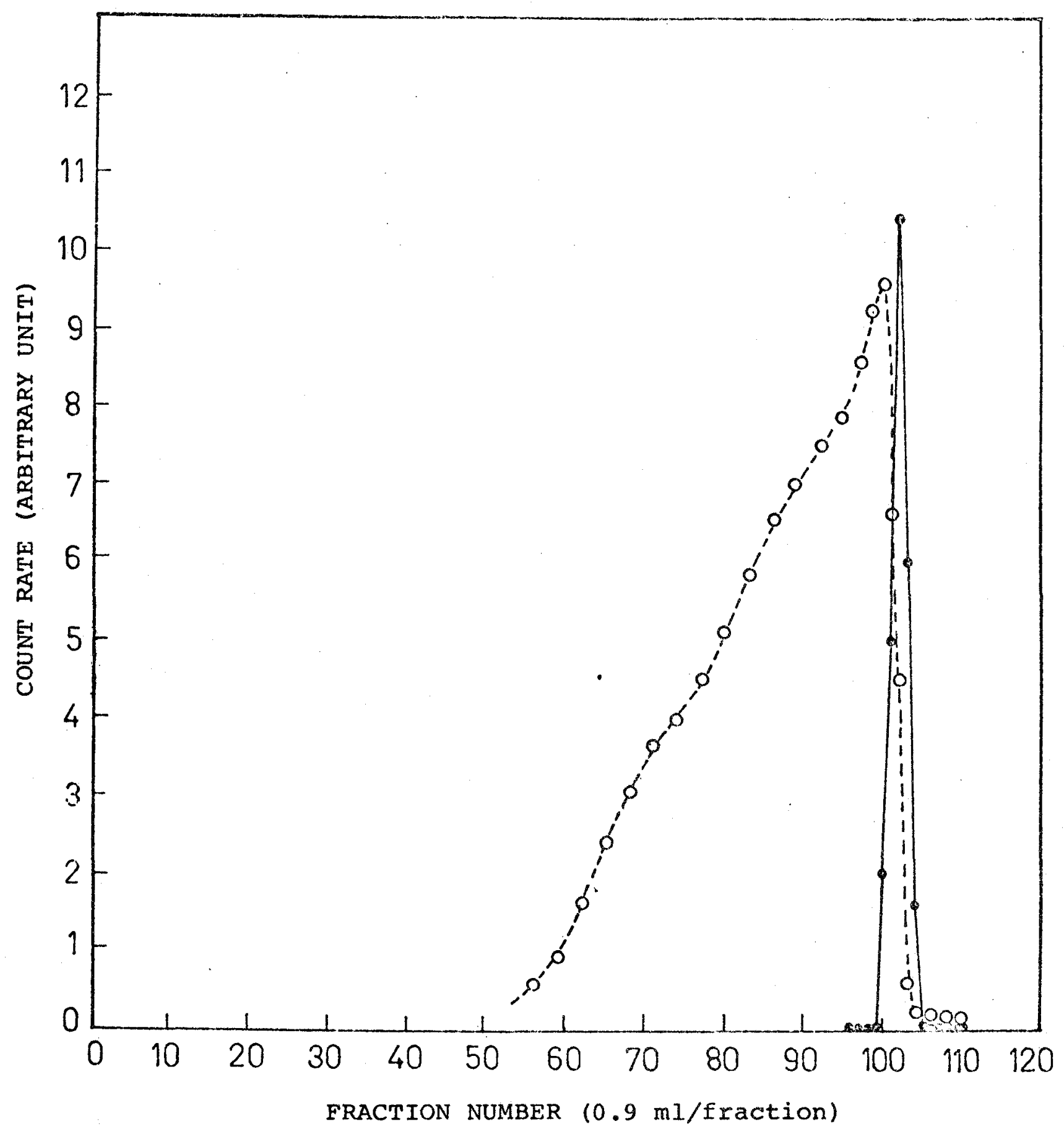

Fig. 10. Elution curve of ${ }^{166}$ Ho + carrier (dashed line) and ${ }^{165}$ Dy tracer (solid line) from Dowex $-50 \times 4$ with $0.5 \mathrm{M} \alpha-\mathrm{HIB}$ as eluant (pH 3.16). 


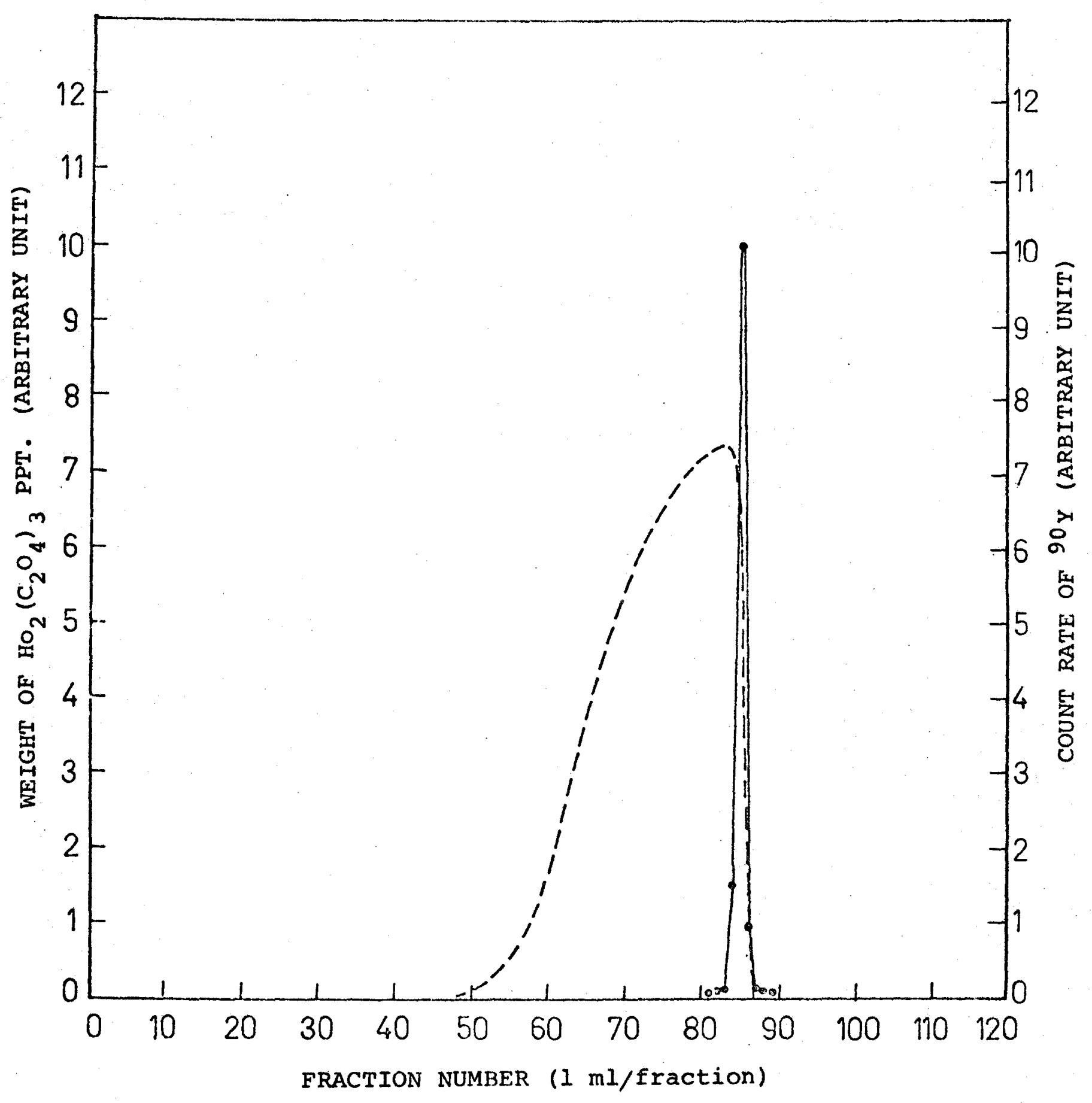

Fig. 11. Elution curve of holmium (dashed line) and ${ }^{90} \mathrm{Y}$ (solid line) from Dowex-50X4 with $0.5 \mathrm{M} \alpha-\mathrm{HIB}$ as eluant (pH 3.16). 
column of Dowex-AGIX10, $100-200$ mesh, $0.6 \mathrm{~cm} \times 6 \mathrm{~cm}$, preconditioned with conc HCl. From the diluted filtrate the holmium hydroxide was precipitated and then dissolved in 3 drops conc $\mathrm{HNO}_{3}$ and diluted to $30 \mathrm{ml}$. About $0.3 \mathrm{ml}$ of wet cation exchange resin of Dowex$50 \times 4$, minus 400 mesh was added to take up the holmium.

In the next step to separate ${ }^{166}$ HO with holmium carrier from the rest of the rare earth fission products, the above resin was transferred to the cation exchange column of Dowex-50x4, minus 400 mesh, $1 \mathrm{~cm} \times 36 \mathrm{~cm}$, prepared as described before. The colimn was eluted with $0.5 \mathrm{M} \alpha-H I B$ solution of $\mathrm{pH} 3.16$ at a flow rate of $\sim 7 \mathrm{ml} / \mathrm{hr}$. Fractions of the eluant were collected at $10 \mathrm{~min}$ intervals and they were examined for the presence of holmium by the addition of saturated oxalic acid. The later fractions containing holmium which might also contain yttrium isotopes were checked with the $\mathrm{NaI}(\mathrm{Tl})$ detector for the $10-\mathrm{hr}{ }^{93} \mathrm{Y}$ activity $\left(\mathrm{E}_{\gamma}=267 \mathrm{keV}\right)$. Fractions free of yttrium activity were combined and holmium oxalate completely precipitated, digested on a hot bath, centrifuged and washed with dilute oxalic acid. Finally the precipitate was taken up with water and filtered onto a glass fibre filter pad, washed with water and 508 alcohol. The samples were dried in a furnace kept at 200$240^{\circ} \mathrm{C}$ for an hour, cooled and then weighed. This was repeated until constant weight was obtained. The chemical form under such condition is known to be $\mathrm{Ho}_{2}\left(\mathrm{O}_{2} \mathrm{O}_{4}\right)_{3} \mathrm{H}_{2} \mathrm{O}^{(83)}$. Chemical yield ratio was calculated and the actual yield was about 608 .

After the chemical yield determination, the samples were carefully placed into the bottom of a $2 \mathrm{cmXl} 5 \mathrm{~cm}$ test-tube and 
$2 \mathrm{ml} 6 \mathrm{M} \mathrm{HCl}$ added for dissolution. It was then counted with a $7.5 \mathrm{~cm} \times 7.5 \mathrm{~cm}$ well-type NaI(Tl) detector coupled to the preamplifier, amplifier and the multichannel analyser system as described before. Each sample was counted several times for $4000 \mathrm{sec}$, background count rates were subtracted after each counting. The resulting ${ }^{166}$ Ho spectra (to be shown in the next chapter) matched identically with standard ${ }^{166}$ Ho spectra. In addition, the half-life measurement also confirmed the purity of the ${ }^{166_{\text {Ho }}}$ samples.

It has to be pointed out that at the time of the separation of $27.2-\mathrm{hr}{ }^{166_{\mathrm{Ho}}}$ from other rare fission products, any primary formed ${ }^{166}$ Ho has undergone decay of several half-lives. Therefore, the ${ }^{166_{\text {Ho }}}$ activity was actually the daughter of $82-\mathrm{hr}{ }^{166} \mathrm{DY}$, which represented the cumulative yield of the 166 mass chain. At the time of the separation of ${ }^{166}$ Dy $-{ }^{166} \mathrm{HO}$, apparently transient equilibrium had been reached and since the two cation exchange columns of the thermal and epi-Sm samples were running side by side, the time difference between the two samples was negligible in the regard of when transient equilibrium was disrupted.

It is also necessary to investigate the possible contamination sources of the very low yield $A=166$ chain from fission of ${ }^{239} \mathrm{Pu}$. The ${ }^{166_{\mathrm{HO}}}$ can be formed from the stable holmium via ${ }^{165_{\text {HO }}}$ $(n, \gamma)$ reaction and ${ }^{166}$ Dy via the double neutron capture of stable 164 Dy. Any contamination due to natural rare earths in the 239 PuAl-alloy or in the aluminum catcher foil could be discovered from the following consideration.

The relative abundances of the rare earths in nature are shown in Table 5. Among the heavier rare earths (> HO), ${ }^{174} \mathrm{Yb}$ 
TABLE 5

Relative Abundances of Rare Earth Elements (84)

$\mathrm{Si}=1 \times 10^{6}$

Sc $\quad 28.0$

$\mathrm{Gd} \quad 0.684$

$\mathrm{Y} \quad 8.9$

$\mathrm{Tb} \quad 0.096$

Ia 2.00

DY $\quad 0.556$

Ce 2.26

Ho $\quad 0.118$

Pr 0.40

Er 0.316

Nd $\quad 1.44$

$\operatorname{Tm} \quad 0.032$

Pm 0

Yb $\quad 0.220$

$\mathrm{Sm} \quad 0.664$

Isu $\quad 0.050$

$\mathrm{Eu} \quad 1.87$ 
and ${ }^{176} \mathrm{Lu}$ are two isotopes with relatively high neutron capture cross-sections. The products of these isotopes from $(n, \gamma)$ reactions are $4.2-\mathrm{d}^{175} \mathrm{Yb}(\gamma$-rays: $113.8 \mathrm{keV}$ and $396.1 \mathrm{keV})$ and $6.8-\mathrm{d}$ ${ }^{177} \mathrm{Lu}$ ( $\gamma$-rays: $113.0 \mathrm{keV}$ and $208.3 \mathrm{keV}$ ). One can therefore estimate the relative activities of ${ }^{166} \mathrm{HO}$, and ${ }^{175} \mathrm{Yb}$ and ${ }^{177} \mathrm{Lu}$ formed for any quantity of rare earths under smilar conditions of irradiation and radiochemical treatments as for the plutonium samples. If significant amount of ${ }^{166}$ Ho were produced by ${ }^{165}$ HO $(n, \gamma)$ and for ${ }^{164}$ Dy $(2 n, \gamma)-{ }^{166}$ Dy $-{ }^{166}$ Ho reactions rather than from fission alone, both ${ }^{175} \mathrm{Yb}$ and ${ }^{177}$ Lu should be detected as described below.

From the elution pattern of the rare earths from the cation exchange resin column with $\alpha-H I B(78)$,ytterbium and lutetium were expected to appear in earlier fractions than holmium in the elution. Fractions before the appearance of holmium were divided into three portions. A few $\mathrm{mg}$ of rare earth carrier $\left(\mathrm{Eu}^{3+}\right.$ ) was added to each portion and then saturated oxalic acid added for the precipitation of rare earth oxalate. The oxalate was counted in a similar way as for holmium oxalate. No 4.2-d ${ }^{175} \mathrm{Yb}$ (396, $114 \mathrm{keV})$ and $6.8-\mathrm{d}{ }^{177_{\mathrm{Lu}}}(113,208 \mathrm{keV})$ activities were detected with the $7.5 \mathrm{~cm} \times 7.5 \mathrm{~cm}$ well-type NaI(TI) counter. Thus the possibility of contamination due to the presence of natural rare earths can be excluded. 


\section{CHAPTER 3}

\section{EXPERIMENTAL RESULTS}

\section{A. Treatment of Gamma-ray Spectra}

The digital gamma-ray spectra recorded were usually plotted and peak positions located for energy alibration. Intensities of gamma-rays were determined by summing the count rates in each channel in the peak and subtracting a background which was estimated by manually fitting a smooth background curve through the spectrum. The count rates of fission products from thermal and epi-cd and from thermal and epi-Sm were compared to give the corresponding mass-yield ratios after necessary decay factor correction. In cases with radiochemical treatments, chemical yield corrections were also made.

B. The Thermal to epi-Cd and the Thermal to epi-Sm Mass-Yield Ratios of Products from Asymmetric Fission

Typical gross gamma-ray spectra of the fission products of ${ }^{239} \mathrm{Pu}$ after $\sim 20$ hours and $\sim 100$ hours cooling periods are presented in Fig. 12 and Fig. 13. Energies of the prominent gamma-rays of each nuclide used for the calculation of the activity ratio are listed in Table 6 . The spectra were consistent with those obtained for thermal neutron fission of ${ }^{235} \mathrm{U}$ by Gordon et al. ${ }^{(64)}$ and Heath (85). 


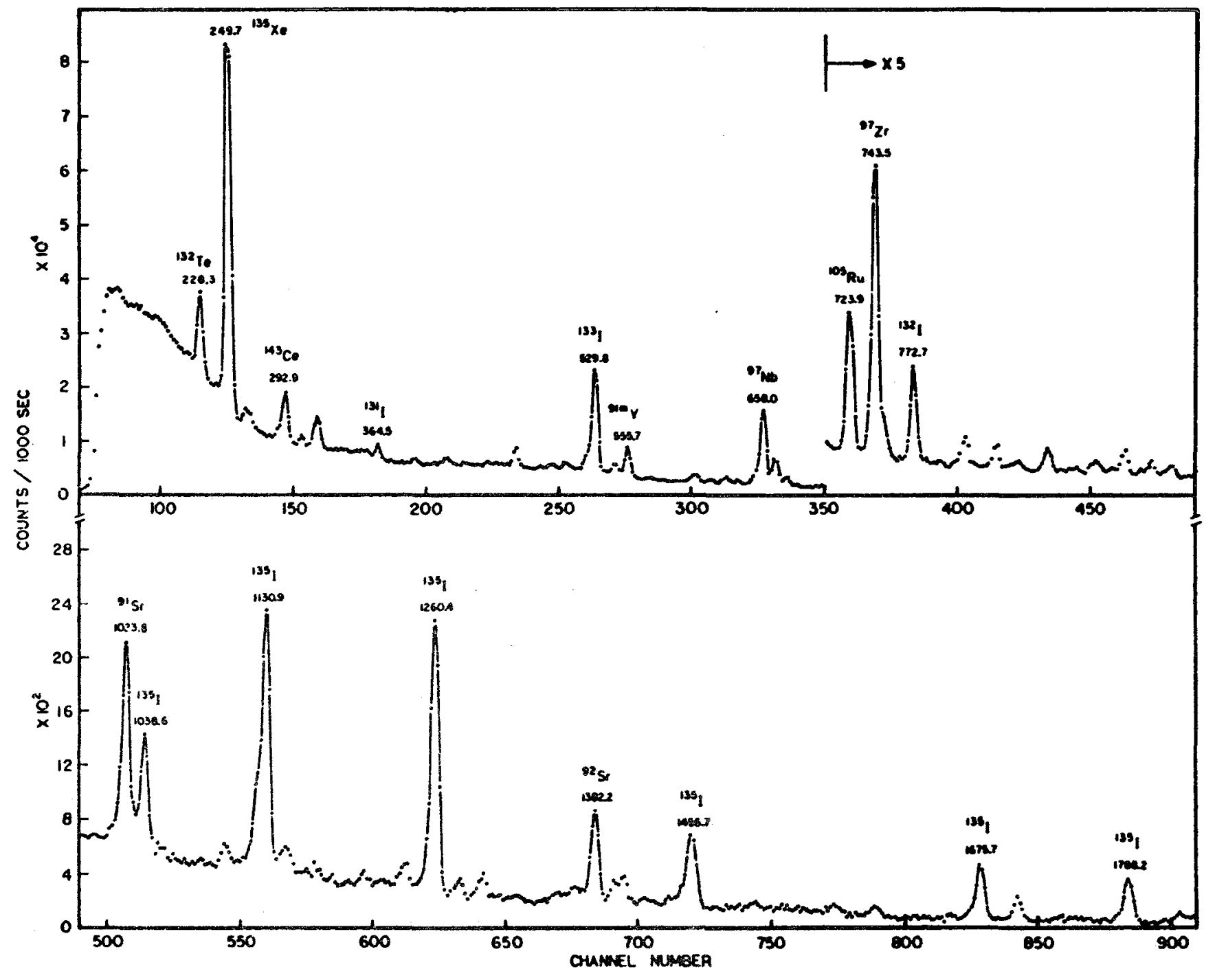

Fig. 12. Gross fission-product $G e(L i)$ spectrum for ${ }^{239} \mathrm{Pu}(n, f), 20$ hours after irradiation. (Peak energies are in $\mathrm{keV}$ ). 


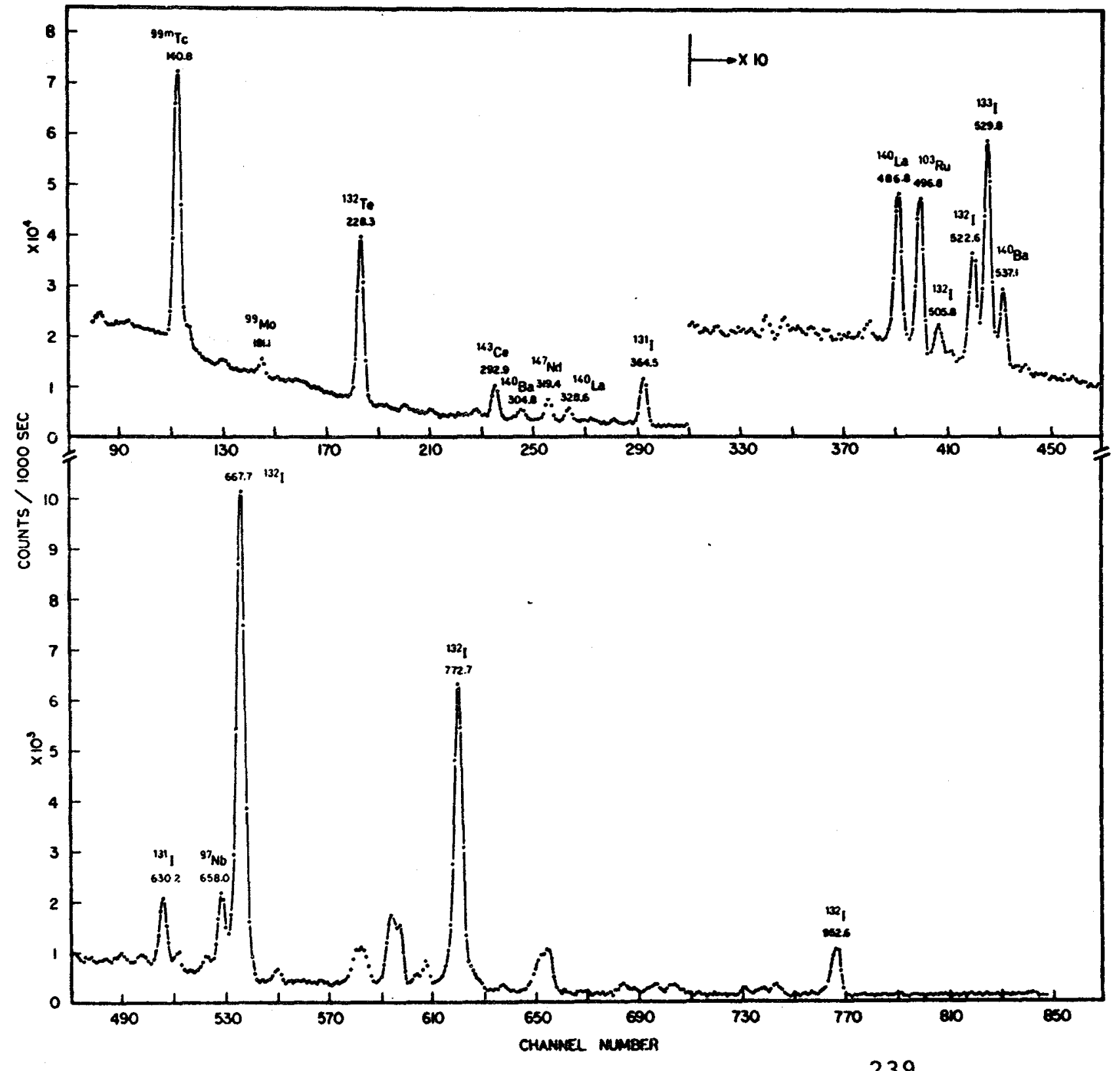

Fig. 13. Gross fission-product Ge(Li) spectrum for ${ }^{239} \mathrm{Pu}(\mathrm{n}, \mathrm{f}), 100$ hours after irradiation. 
TABIE 6

Prominent Gamma-rays in Gross Fission

Products Ge(Li) Spectrum

Nuclide

Half-life
Prominent $\gamma$-rays

(keV)

$\begin{array}{ccc}85 \mathrm{~m}_{\mathrm{Kr}} & 4.4-\mathrm{hr} & 151.2 \\ 9{ }^{1} \mathrm{Sr} & 9.7-\mathrm{hr} & 1024.3 \\ 91 \mathrm{~m}_{\mathrm{Y}} & 50-\mathrm{min} & 555.6 \\ 92 \mathrm{Sr} & 2.7-\mathrm{hr} & 1384.0 \\ 97 \mathrm{Zr} & 17-\mathrm{hr} & 743.5 \\ 97 \mathrm{Nb} & 72-\mathrm{min} & 658.0 \\ 99 \mathrm{~m}_{\mathrm{Tc}} & 6.0-\mathrm{hr} & 140.5 \\ 103 \mathrm{Ru} & 40-\mathrm{d} & 496.9 \\ 105 \mathrm{Ru} & 4.4-\mathrm{hr} & 723.9 \\ 131 \mathrm{I} & 8.05-\mathrm{d} & 364.5 \\ 132 \mathrm{Te} & 78-\mathrm{hr} & 228.3 \\ 132 \mathrm{I} & 2.3-\mathrm{hr} & 667.7,772.8 \\ 133_{\mathrm{I}} & 21-\mathrm{hr} & 529.8 \\ 135 \mathrm{I} & 6.7-\mathrm{hr} & 1260.4 \\ 135 \mathrm{Xe} & 9.2-\mathrm{hr} & 249.6 \\ 140 \mathrm{Ba} & 12.8-\mathrm{d} & 304.8,537.2 \\ 140 \mathrm{La} & 40.2-\mathrm{hr} & 328.8,487.0 \\ 143 \mathrm{Ce} & 33-\mathrm{hr} & 293.3 \\ 147 \mathrm{Nd} & 11.1-\mathrm{d} & 319.4\end{array}$


Any minor components overlapping with the prominent gammarays were resolved. Peak areas of the prominent peaks were estimated by summing the count rates in the peak channel and subtracting a smooth background which was fitted to the spectrum. The intensities of the high-yield fission products for thermal and epi-Cd fissions, and for thermal and epi-Sm fissions, corrected for any half-life effect, were directly compared.

Table 7 shows the ratio from each run of the experiments, and the errors quoted are solely due to counting statistics. No fluctuations were observed in these activity ratios.

The thermal and epi-thermal samples were counted at identical geometry, and any sequential beta decays of the gamma transitions were allowed to go to completion or otherwise have reached the state of transient equilibrium before counting. From the mass-yield distribution curve we also see that the total yields of products from asymetric fission virtually sum up to $200 \%$. It is therefore possible to assume that the activity ratios represent the mass-yield ratios and hence the relative number of fission events in the thermal and epi-thermal fissions of ${ }^{239} \mathrm{Pu}$. Thus it is implied in these data that there are no significant variations in the mass yields of asymmetric products for epi-Cd or epi-Sm as compared with thermal neutron fissions.

c. The Thermal to epi-Sm Mass-Yield Ratios of ${ }^{72} \mathrm{zn},{ }^{112} \mathrm{Pd}$ and ${ }^{115} \mathrm{Cd}$ Typical experimental spectra of ${ }^{72} \mathrm{zn}$ (Obtained with 
TABLE 7

Activity Ratios of Fission Products for Thermal to epiThermal Fission of ${ }^{239} \mathrm{Pu}$ from Nondestructive Ge(Li) Measurements

$\begin{array}{clllllll}\begin{array}{c}\text { Experiment } \\ \text { Number }\end{array} & 1 & 2 & 3 & 4 & 5 & 6\end{array}$

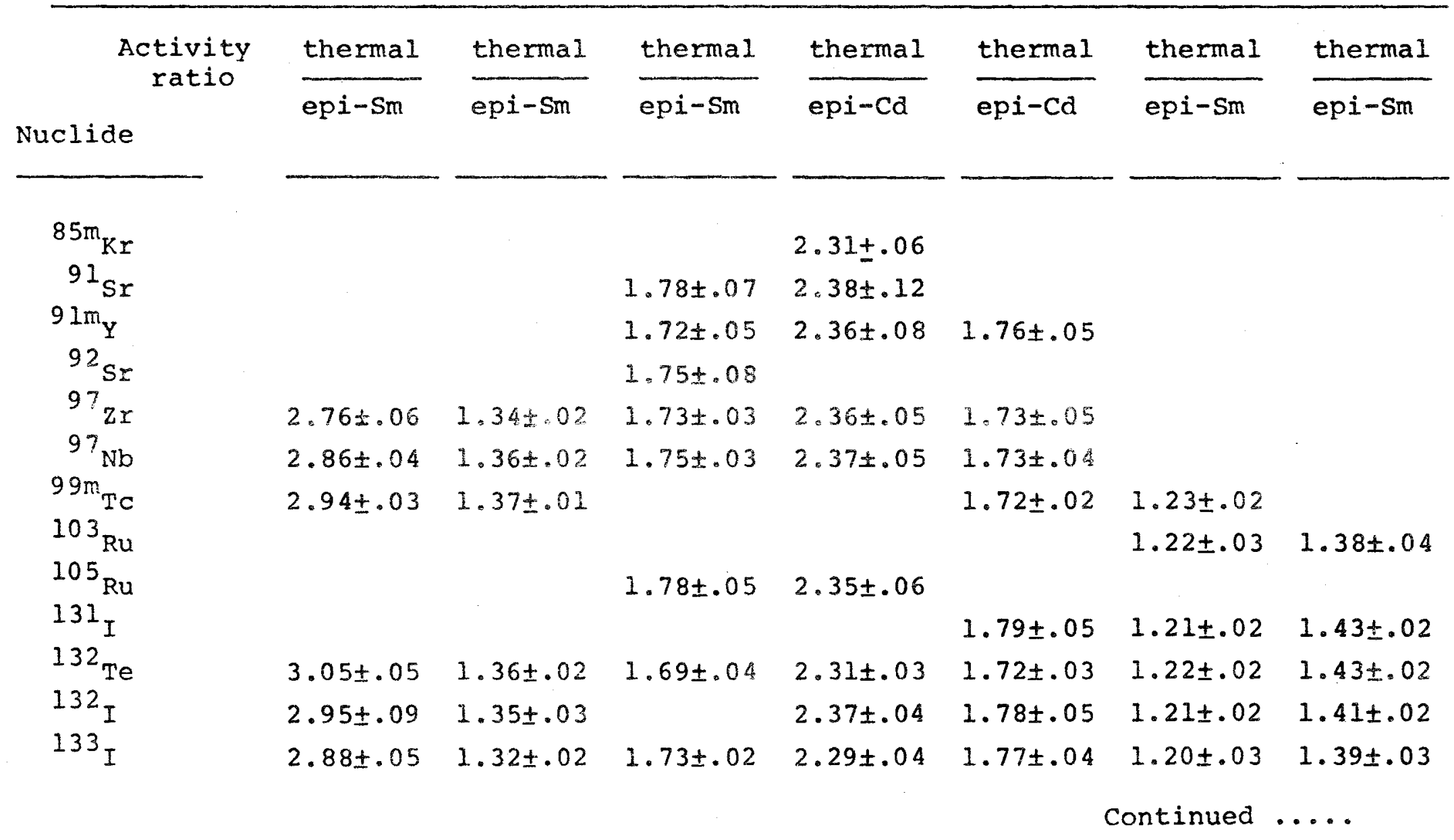


TABLE 7 (Continued)

Experiment Number

Nuclide

\begin{tabular}{l}
$135 \mathrm{I}$ \\
$135_{\mathrm{Xe}}$ \\
$140 \mathrm{Ba}$ \\
$140 \mathrm{Ia}$ \\
$143_{\mathrm{Ce}}$ \\
$147 \mathrm{Nd}$ \\
\hline
\end{tabular}

Weighted Average
3

4

5

6

7

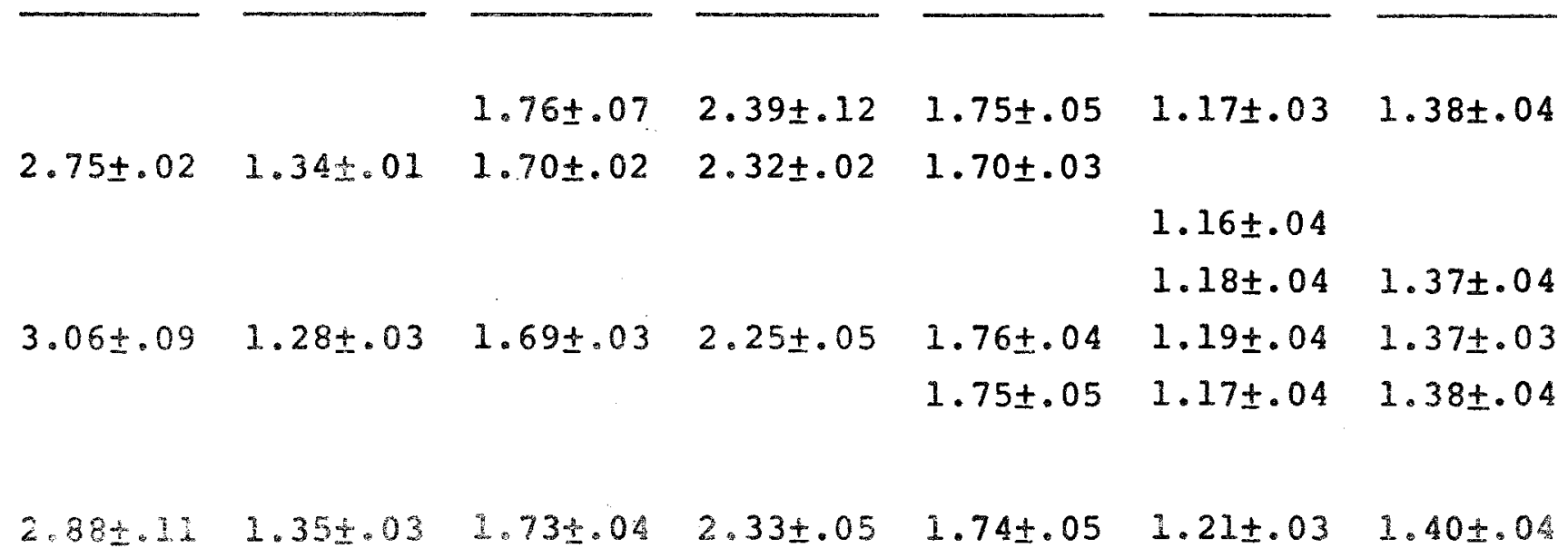

$2.38+.121 .35+.03$ 
$\mathrm{NaI}(\mathrm{T} 1)$ detector) and ${ }^{112} \mathrm{Pd}$ and ${ }^{115} \mathrm{Cd}$ (obtained with Ge(Li) detector) are shown in Figs. 14,15 and 16 respectively. Energies of the relevant gamma peaks were calibrated as closely as possible. Countings carried out at several intervals for each sample also allowed to measure the half-life, and hence to determine whether there were any interferences to these gamma-rays or not.

The count rates obtained were corrected for decay effects and chemical yields and then compared. Results of the activity ratios, i.e. activity of product from thermal fission of ${ }^{239} \mathrm{pu}$ to activity from epi-Sm fission, of the above isotopes are presented in Table 8 . Since the relative number of fission events in the thermal and epi-Sm samples were given by the activity ratios of the high-yield fission products as we have shown in the last section, the thermal to epi-Sm mass-yield ratios for these isotopes were also computed. We recall that the definition of " $R$ " value, discussed in Chapter 1, as measure of the mass-yield ratios of asymmetric to symmetric fission products as

$$
R=\frac{\left(A_{\text {asym }} / A_{\text {sym }}\right) \text { resonance }}{\left(A_{\text {asym }} / A_{\text {sym }}\right) \text { thermal }}
$$

Obviously, the mass-yield ratios we obtained for $72 \mathrm{zn}, 112 \mathrm{Pd}$ and ${ }^{115} \mathrm{Cd}$ are actually equivalent to the " $R$ " values. 


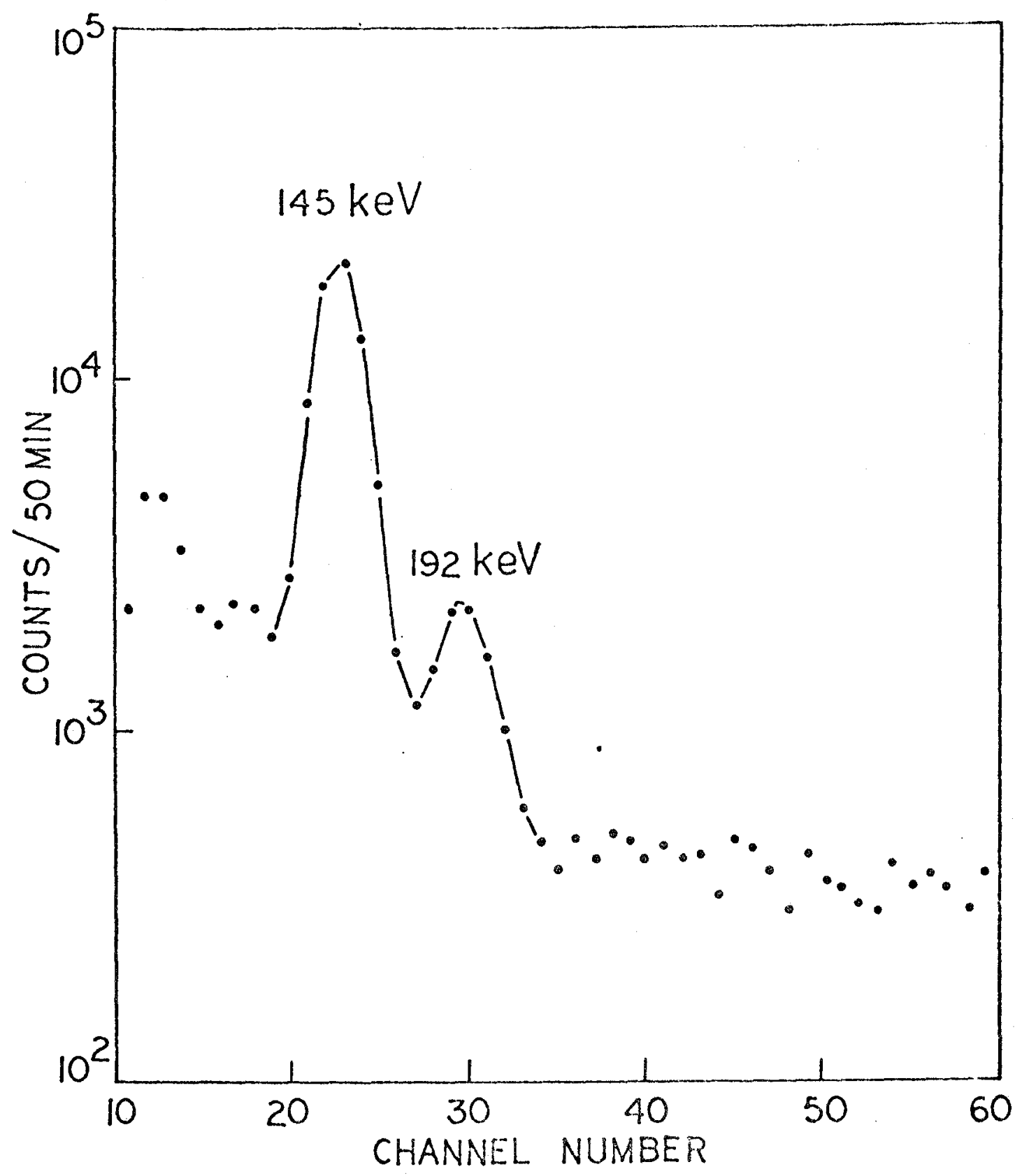

Fig. 14. NaI(TI) gamma-ray spectrum of ${ }^{72} \mathrm{zn}$. 


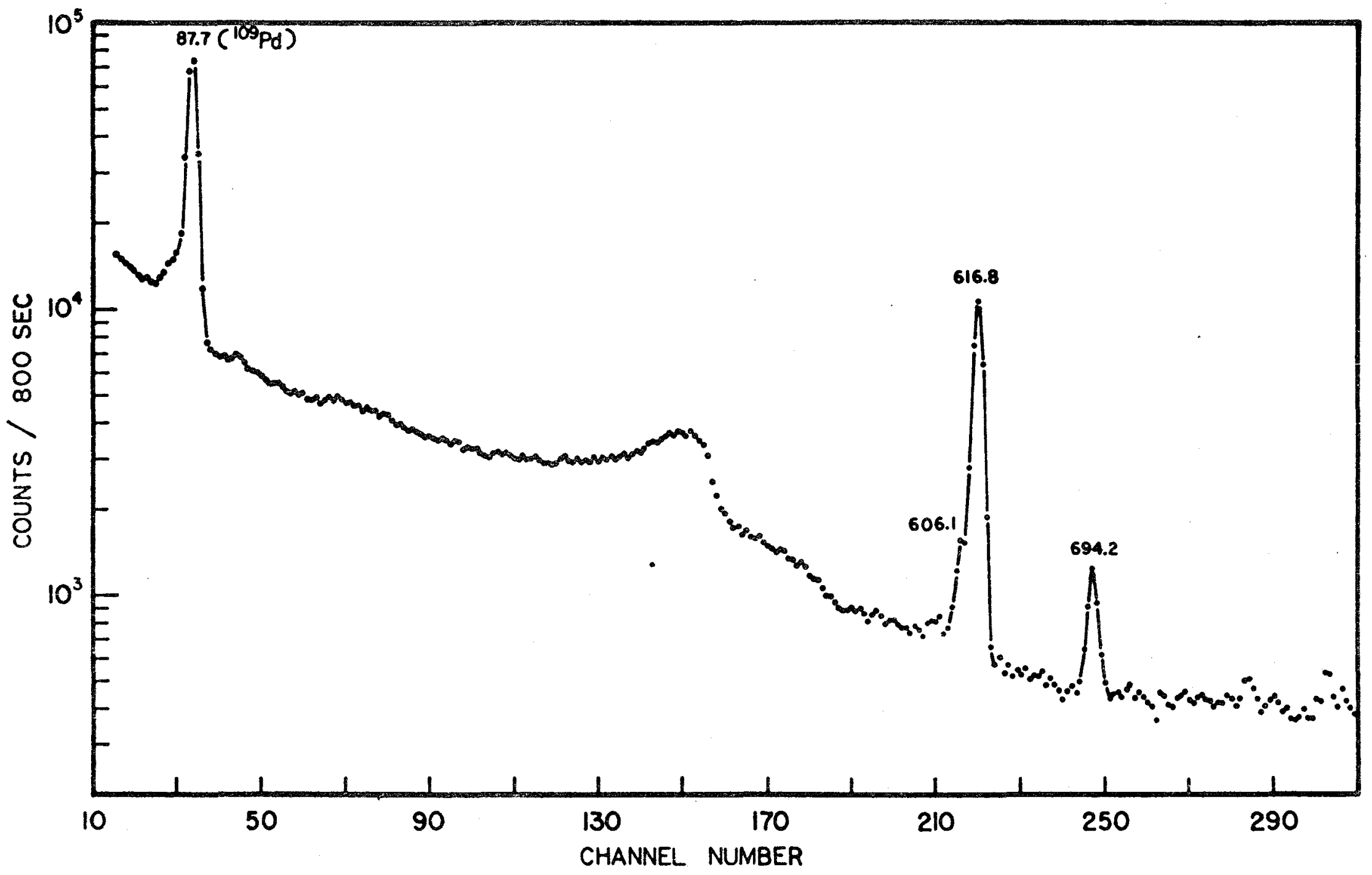

Fig. 15. The Ge(Li) gamma-ray spectrum of ${ }^{112} \mathrm{Pd}-{ }^{112} \mathrm{Ag}$.

$\vec{I}$ 


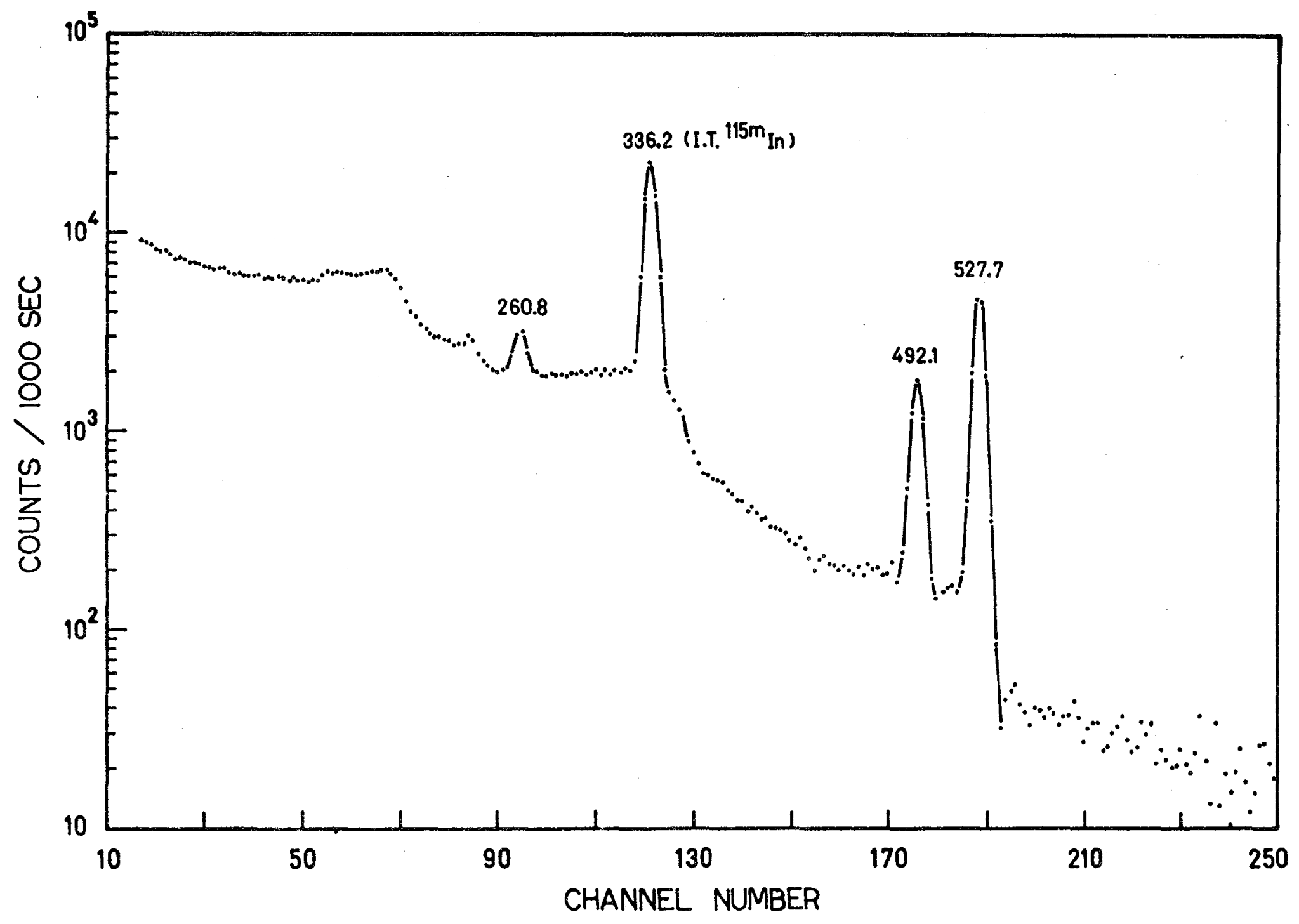

Fig. 16. The Ge(Li) gamma-ray spectrum of ${ }^{115} \mathrm{Cd}-{ }^{115 \mathrm{~m}} \mathrm{In}$. 
Measured and Normalized Mass-Yield Ratios

for Thermal to epi-Sm Fission of $239 \mathrm{Pu}$

$\left({ }^{72} \mathrm{zn},{ }^{112} \mathrm{Pd}\right.$, and $\left.{ }^{115} \mathrm{Cd}\right)$.

\begin{tabular}{|c|c|c|c|c|c|}
\hline \multirow[b]{2}{*}{ Nuclide } & \multicolumn{2}{|c|}{$\begin{array}{c}\text { Measured } \\
\text { Activity Ratio } \\
\end{array}$} & \multicolumn{2}{|c|}{$\begin{array}{l}\text { Normalized mass- } \\
\text { yield ratio (R) }\end{array}$} & \multirow[t]{2}{*}{$\begin{array}{l}\text { Average } \\
\text { o decre }\end{array}$} \\
\hline & $\# 1$ & $\# 2$ & $\# 1$ & \#2 & \\
\hline $72 \mathrm{zn}$ & $4.20 \pm 0.10$ & $1.91 \pm 0.03$ & $1.46 \pm 0.07$ & $1.42 \pm 0.04$ & $30 \pm 1$ \\
\hline${ }^{112} \mathrm{Pd}$ & $3.96 \pm 0.22$ & $1.70 \pm 0.04$ & $1.38 \pm 0.09$ & $1.26 \pm 0.04$ & $24 \pm 3$ \\
\hline${ }^{115} \mathrm{Cd}$ & $5.54 \pm 0.20$ & $2.45 \pm 0.11$ & $1.92 \pm 0.10$ & $1.82 \pm 0.09$ & $46 \pm 1$ \\
\hline
\end{tabular}


D. The Thermal to epi-Cd and Thermal to epi-Sm Mass-Yield Ratios of ${ }^{77} \mathrm{Ge}$ and ${ }^{77} \mathrm{As}$

Presented in Figs. 17. and 18 are the Ge(Li) spectra of ${ }^{77} \mathrm{Ge}$ and $77 \mathrm{As}$ respectively. Gamma lines due to impurities are indicated. Gamma peaks used for the activity measurements are $239.3 \mathrm{keV}$ for $77_{\mathrm{As}}$, and $264.4 \mathrm{keV}$ and the $211.0-215.6 \mathrm{keV}$ doublet for ${ }^{77} \mathrm{Ge}$. Countings were usually followed through several half-lives. Both the energy and half-life calibration of these gamma peaks showed no interference from impurity activities of other isotopes.

The activity ratios were computed and given in Table 9, in a similar way as for the ${ }^{72} \mathrm{zn}$ group. The " $R$ " values were also obtained.

E. The Thermal to epi-Sm Mass Yield Ratios of ${ }^{166}$ Ho - ${ }^{166}$ Dy A spectrum of ${ }^{166}$ Ho obtained with the $7.5 \mathrm{~cm} \times 7.5 \mathrm{~cm}$ well-type NaI(TI) detector is shown in Fig. 19, with room background and counter noise self-subtracted. The activity ratios and " $R$ " values were obtained after necessary half-life and chemical yield corrections and presented in Table 10. These values are designated for ${ }^{166}$ DY $-{ }^{166}$ Ho because of the equilibrium of the two isotopes at the stage of chemical separation.

F. Cadmium Ratios and Fast Neutron Fission Contribution The measured cadmium ratios of the two in-core positions usually employed in the irradiations of this work were in 


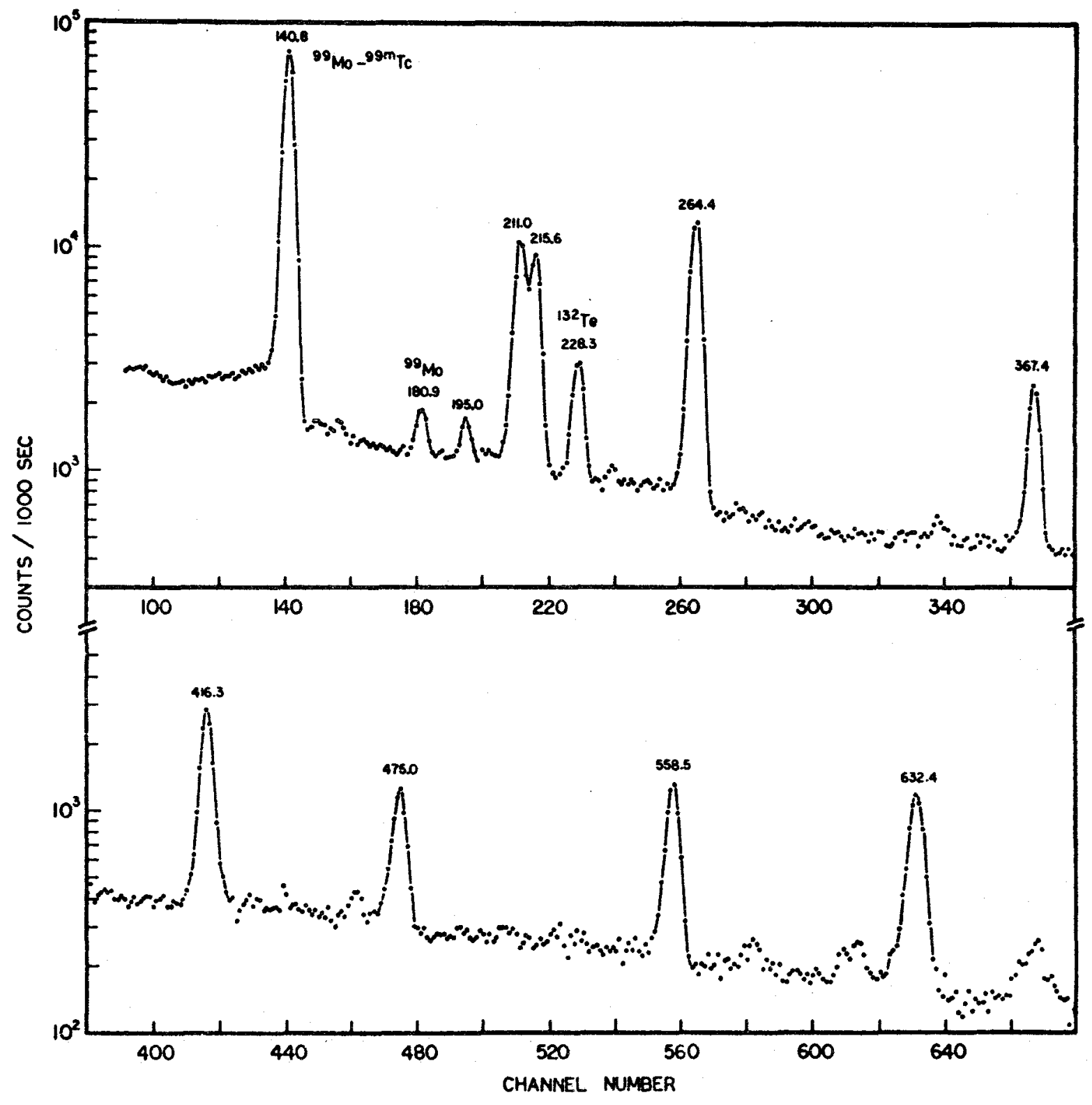

Fig. 17. The Ge(Li) gamma-ray spectrum of ${ }^{77} \mathrm{Ge}$. 


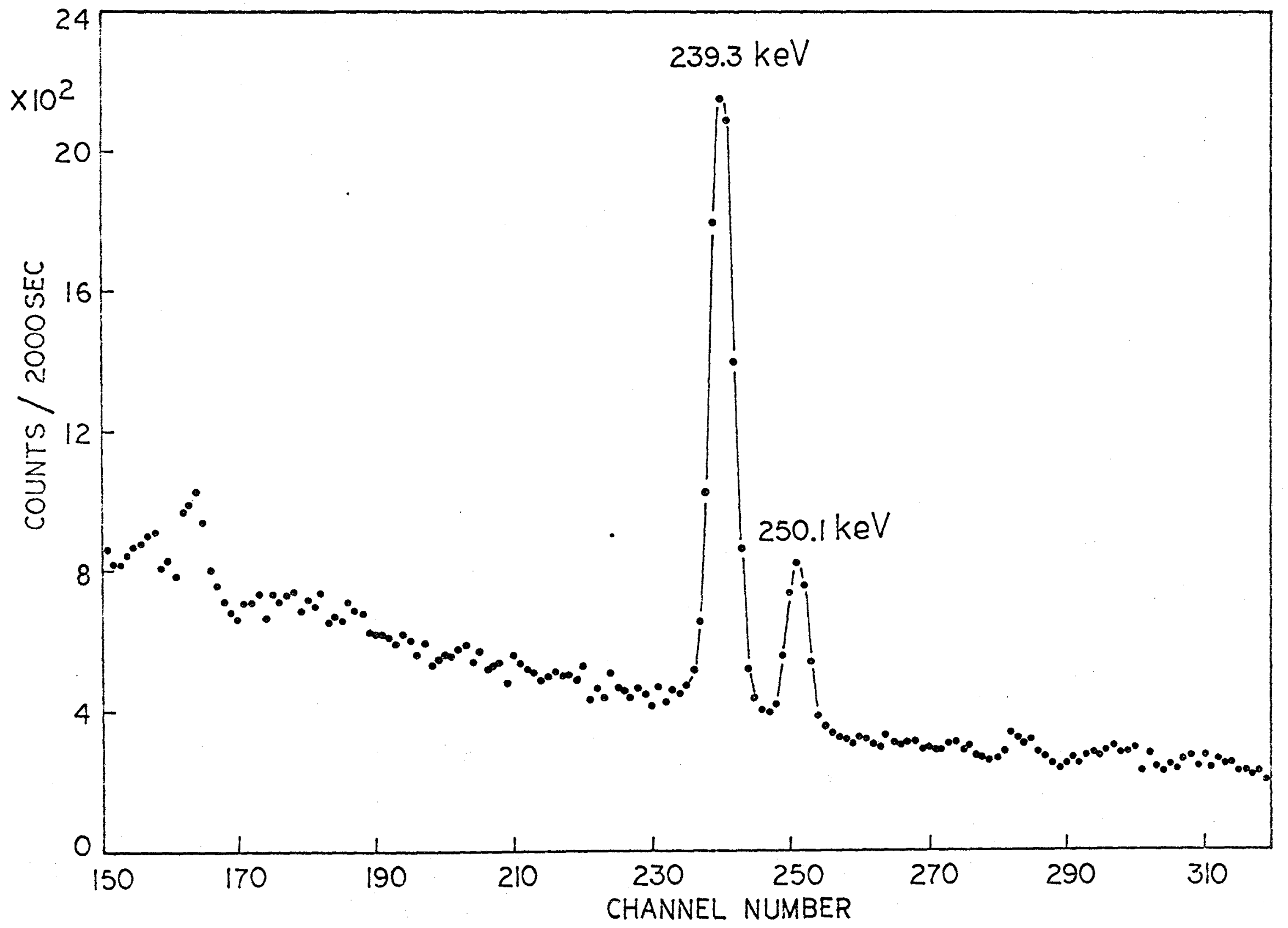

Fig. 18. The Ge(Li) gamma-ray spectrum of ${ }^{77} \mathrm{As}$. 
TABLE 9

Measured and Normalized Mass-Yield Ratios for Thermal to Epi-thermal Fission of ${ }^{239} \mathrm{Pu} \quad\left({ }^{77} \mathrm{Ge}\right.$ and $\left.{ }^{77} \mathrm{As}\right)$

-

Measured Activity Ratio

$\longrightarrow$

Nuclide

\#3.

thermal epi-Sm

\#4

thermal epi-cd

\#5 $\frac{\text { thermal }}{\text { epi-cd }}$

$\# 3$

$1.08 \pm 0.05$

$1.07 \pm 0.05$

${ }^{77} \mathrm{Ge}$

$1.87 \pm 0.08 \quad 2.50 \pm 0.10$

$1.79 \pm 0.08$

$1.03 \pm 0.05$ 


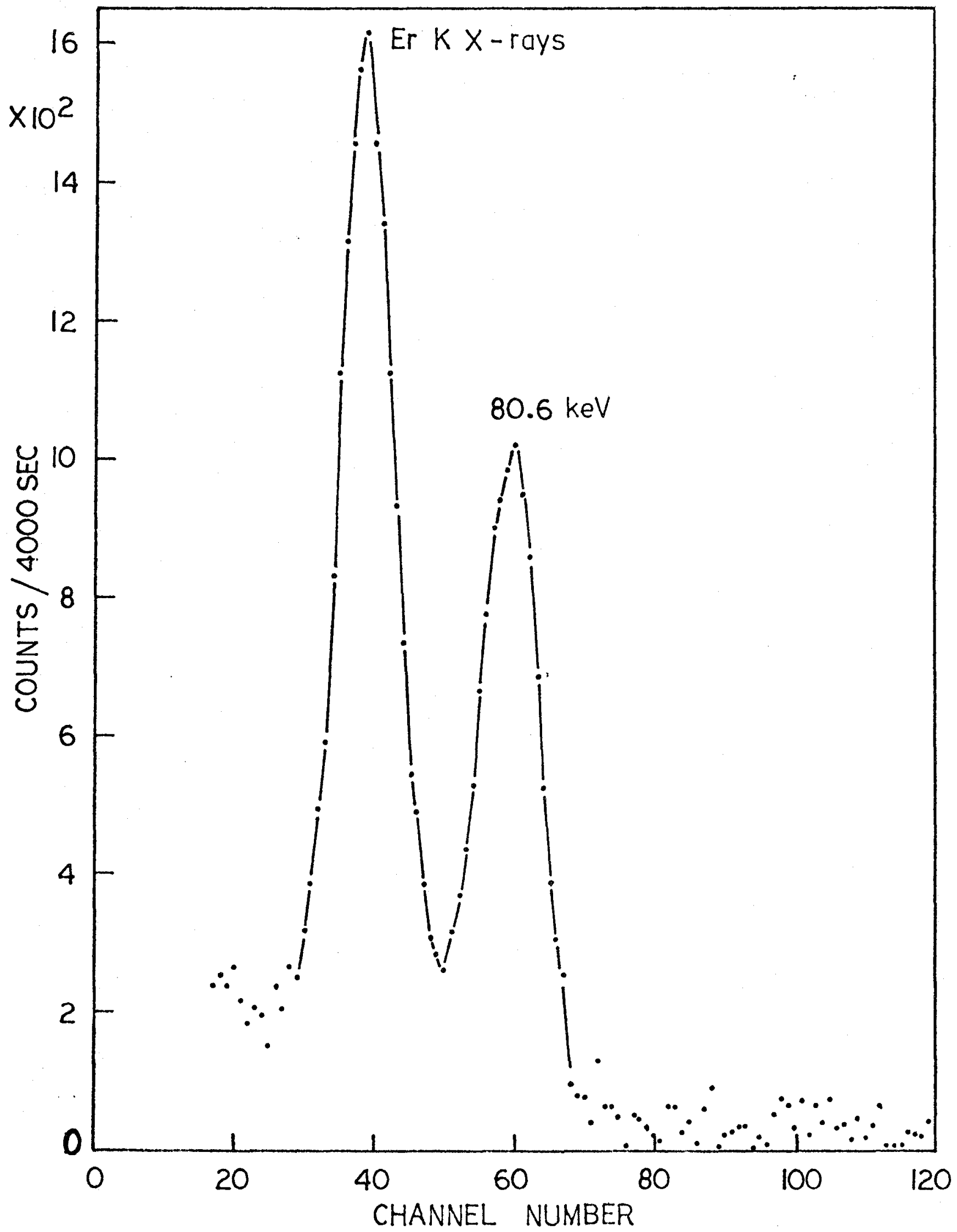

Fig. 19. The NaI(T1) gamma-ray spectrum of ${ }^{166}$ Ho. 


\section{TABLE 10}

Measured and Normalized Mass-Yield

Ratios for Thermal and epi-Sm Fission of ${ }^{239} \mathrm{Pu}\left({ }^{166} \mathrm{DY}-{ }^{166} \mathrm{HO}\right)$.

Experiment \# Measured Activity Normalized mass-

Average $\%$ Ratio yield ratio (R)

6

$$
1.68 \pm 0.06 \quad 1.40 \pm 0.07
$$

7
$2.13 \pm 0.07$
$1.50 \pm 0.07$

$31 \pm 1$ 
agreement within the experimental error. They were found to be $14.0 \pm 0.8$ for $\mathrm{Mn}$ and $1.98 \pm 0.10$ for $\mathrm{Au}$.

As mentioned in the previous chapter, a known amount of thorium metal was irradiated under similar condition as for the epi-sm ${ }^{239} \mathrm{Pu}$ sample. The thorium sample showed mostly activity of $27.4-d{ }^{233} \mathrm{~Pa}$ formed by the activation of ${ }^{232}$ Th followed by beta decay. However, activities due to fission products from 232 Th (fast $n, f$ ) reaction could also be identified in the highresolution Ge(Li) spectrum shown in Fig. 20. The $364.3 \mathrm{keV}$ and $486.8 \mathrm{keV}$ gamma lines associated with ${ }^{13 I \mathrm{I}}$ and ${ }^{140} \mathrm{La}$ respectively are indicated. Comparison of the results with the epi-Sm sample counted with the same Ge(Li) detector at identical geometry is shown in Table 11. With these data and taking the fast-neutron fission cross-sections of ${ }^{232} \mathrm{Th}$ and ${ }^{239} \mathrm{Pu}$, and the spectrum of fission neutronsinto consideration, it was estimated that $\sim 18$ of the total fission events of epi-Sm ${ }^{239} \mathrm{Pu}$ were induced by fast neutrons. 


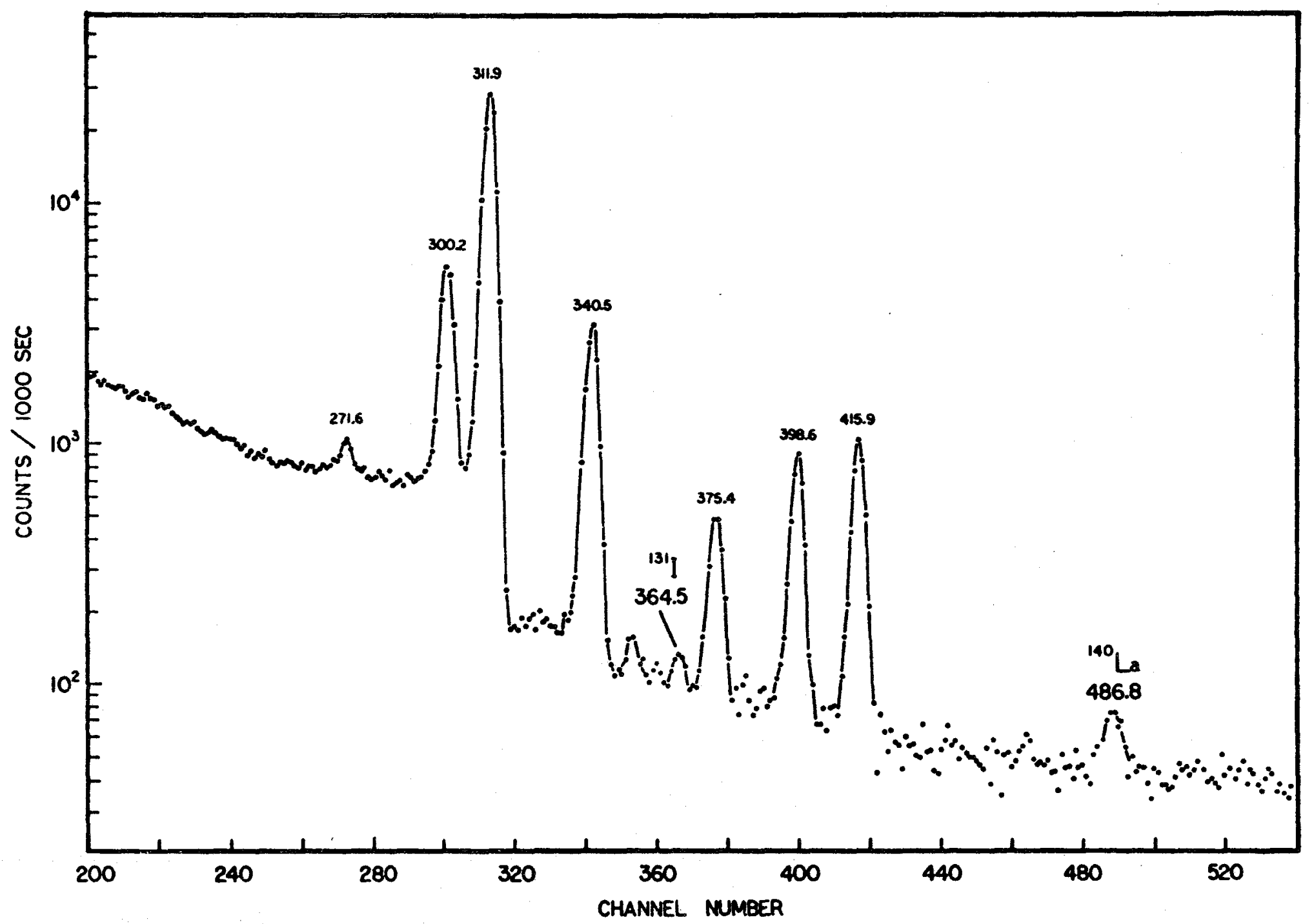

Fig. 20. Ge (Li). gamma-ray spectrum of epi-Sm neutron irradiated ${ }^{232} \mathrm{Th}$. Gamma-rays from fission products $\left({ }^{131} \mathrm{I}\right.$ and $\left.{ }^{140} \mathrm{La}\right)$ are indicated. 
TABLE 11

Fast-Neutron Fission Contribution Measurement

${ }^{239} \mathrm{Pu}(\mathrm{epi}-\mathrm{Sm}) \quad{ }^{232} \mathrm{Th}$

Sample weight

$1.1 \mathrm{mg}$

$24.5 \mathrm{mg}$

Fast-neutron fission crosssection

2 barns

$0.1-0.2$ barns

Corrected count rate of ${ }^{131} I$

36000

120

( 8 cumulative mass yield)*

$(3.88)$

(1.28)

Corrected count rate of ${ }^{140} \mathrm{La}$

13000

130

( 8 cumulative mass yield) *

$(5.58)$

$(6.28)$

* From reference (29). 


\section{CHAPTER 4}

\section{DISCUSSION}

A. Relative Fission Rates of the Low-Energy Resonances in Thermal, epi-Cadmium and epi-Samarium Fissions of ${ }^{239} \mathrm{Pu}$

It is of interest to investigate the relative fission rates of the low energy resonances in the thermal, epi-Cd and epi-sm neutron fissions of ${ }^{239} \mathrm{Pu}$. Such information is useful in the analysis of the present experimental results. Relatively crude estimations were carried out because only semiquantitative interpretations were possible.

It has been shown from the irradiation of metallic thorium that the contribution of fission induced by fission spectrum neutrons in the epi-sm fission ${ }^{239} \mathrm{Pu}$ sample was $\sim 1 \frac{8}{6}$ of the total fission events. In addition, Cuninghame et al. (86) reported that the peak-to-valley mass-yield ratio in terms of ${ }^{99} \mathrm{Mo} /{ }^{113} \mathrm{Ag}$ for $1 \mathrm{MeV}$ and $6 \mathrm{Mev}$ neutron-induced fission of ${ }^{239} \mathrm{Pu}$ were $71 \pm 9$ and $16.7 \pm 2$ respectively compared with 86 for thermal neutroninduced fission. Fission contribution due to fission spectrum neutronsmay therefore be neglected in considering the mass yield variations in the thermal and epi-thermal fissions. The measured cadmium ratio for the irradiation positions was $14.0 \pm 0.8$ for manganese and $1.98 \pm 0.10$ for gold. According 
to the Westcott convention, the effective cross-section $\hat{\sigma}$ is given in terms of the $2200 \mathrm{~m} / \mathrm{sec}$ value $\sigma_{0}$ by the relation

$$
\hat{\sigma}=\sigma_{0}(g+r s)
$$

where $g$ and $s$ are factors compiled in reference (87), and $r$ is a measure of the proportion of epithermal to thermal neutrons of the reactor neutron spectrum. The value of $r$ may be deduced from the cadmium ratio measurement. Using a thin detector having $g \approx 1$ the formula applicable is

$$
R_{C d}=\frac{1+r s}{r\left(s+4 \sqrt{k T / \pi E_{C d}}\right)}
$$

where $\mathrm{E}_{\mathrm{Cd}}=$ cadmium cut off energy, assumed to be $0.67 \mathrm{eV}$ for

a $1 \mathrm{~mm}$ thick cadmium cylinder

$k=$ Boltzman constant

and $T=$ reactor neutron temperature, $\sim 35^{\circ} \mathrm{C}$.

The $r$ value evaluated with the above formula was

0.059. Hence the neutron spectrum can be approximately represented according to Beckurtz and Wirtz ${ }^{(88)}$ by

$$
\Phi(E)=c\left[\frac{E}{(k T)^{2}} e^{-E / k T}+0.059 \frac{\Delta(E / k T)}{E}\right]
$$

where $c$ is a constant characteristic of the total flux and $\Delta(E / k T)$ is the "joining function" of the thermal flux, represented by the first term, and the epi-thermal flux, expressed as a $1 / E$ function in the second term.

The joining function $\Delta(E / k T)$ can be either the unit step $E=\mu \mathrm{kr}$ or the function

$$
1 /\left[1+(\mu k T / E)^{16}\right]
$$


where $\mu$ is an integer multiple. Westcott ${ }^{(87)}$ has chosen $\mu=5$ as a reasonable fit to the theoretical spectra computed for weakly absorbing $\mathrm{D}_{2} \mathrm{O}$ media. Since the "joining function" has been observed to depend rather weakly on the properties of the moderator (88) ${ }^{(8)} \mu=5$ was adopted for the present calculation.

Figure 21 shows the computed neutron spectrum with $c=2 \times 10^{13}$.

Fission rates of ${ }^{239} \mathrm{Pu}$ as a function of energy can be computed if the fission cross-section function and the neutron spectrum are known. For a ${ }^{239} \mathrm{Pu}$ sample with $\mathrm{N}_{\mathrm{o}}$ nuclei of ${ }^{239} \mathrm{Pu}$ per $\mathrm{cm}^{2}$, the number of interactions is

$$
N_{I}(E)=\Phi(E)\left[1-e^{-N_{0} \sigma_{t}(E)}\right]
$$

where $\sigma_{t}(E)$ is the total cross-section. The fission rate is therefore given as

$$
R_{f}(E)=\frac{\sigma_{f}(E)}{\sigma_{t}(E)}\left[1-e^{-N_{0} \sigma_{t}(E)}\right] \Phi(E)
$$

where $\sigma_{f}(E)$ is the fission cross-section. For $N_{0} \sigma_{t}(E) \ll 1$, this formula reduces to

$$
R_{f}(E) \simeq N_{O} \sigma_{f}(E) \Phi(E)
$$

This approximation is valid for the present calculation with $N_{0} \sigma_{t}$ always less than 0.1 and error as high as about 108 permissible for the present purpose.

It is more useful to evaluate fission rates due to 


$$
\text { D. }
$$


different. resonances, if the ${ }^{239} \mathrm{pu}$ fission cross-section can be described in detail in terms of a full set of individual level parameters. According to Lynn ${ }^{(51)}$ this is possible for low energy neutron fissions of ${ }^{239} \mathrm{Pu}$ based on the results of extensive studies by various authors (see (51)). These investigations indicate that the interfernces of the narrow $1^{+}$levels (constitute about three quarters of the $S$-Wave levels) and the widely spaced $0^{+}$levels are negligible. Hence, the total fission cross section $\sigma_{f}(E)$ can be expressed in terms of the sum of single-level Breit-Wigner terms as

$$
\sigma_{f}(E)=\sum_{i} \sigma_{f i}(E)=\sum_{i} 4 \pi x^{2} \frac{{ }{ }_{J i}{ }{ }_{n i}{ }^{\Gamma} f i}{4\left(E-E_{O i}\right)^{2}+\Gamma_{i}}
$$

where subscript $i$ refers to the resonance centered at energy $E_{o i} \star \star$ is the de Broglie wavelength of the relative motion of neutron and target divided by $2 \pi, \Gamma_{n}$ and $\Gamma_{f}$ are the neutron and fission widths respectively, $\Gamma_{t}$ is the total width and $g_{J}$ is a statistical spin factor depending on the total angular momentum $J$ of the compound state. These parameters for resonances up to $260 \mathrm{eV}$ as derived by various authors have been compiled in reference and they are used in the subsequent calculations.

The fission rate due to resonance $i$ is given by

$$
R_{i}=\int_{E_{1}}^{E_{2}} N_{0} \sigma_{f i}(E) \Phi(E) d E
$$

where $E_{1}$ and $E_{2}$ are two limits chosen to give sufficient accuracy in the integration. The $R_{i}$ values were computed 
using different sets of input parameters due to various authors.

Calculations of the relative fission rates of different resonances for the epi-Cd and epi-Sm samples are more complicated. The Cd-and Sm-filtered neutron spectrum as seen by the ${ }^{239} \mathrm{Pu}$ samples is approximately

$$
\Phi^{\prime}(E)=\Phi(E) e^{-n \sigma} a b s(E)
$$

where $\Phi(E)$ is the unperturbed neutron spectrum, $e^{-n \sigma_{a b s}(E)}$ is the transmission factor, $\mathrm{n}$ equals the number of $\mathrm{Cd}$ or $\mathrm{Sm}$ atoms per $\mathrm{cm}^{2}$ used in shielding, and $\sigma_{\text {abs }}(\mathrm{E})$ is the absorption cross-section of $\mathrm{Cd}$ or $\mathrm{Sm}$ as a function of neutron energy. The fission rate equation for individual resonance then becomes

$$
R_{i}=\int_{E_{2}}^{E_{2} N_{0} \sigma_{f i}(E) \Phi(E) e^{-n \sigma a b s}(E)} d E \text {. }
$$

From the cross-section curve for cadmium (89), we see that only a single-level Breit-Wigner fit for the resonance at $0.178 \mathrm{eV}$ is required for the cross-section expression to be used in the transmission factor. This is given as follows $(90)$ :

$$
n \sigma_{a b s}(E)=0.0050762 \ell_{0} /\left\{\sqrt{ } E\left[4 \cdot(E-0.178)^{2}+0.013112\right]\right\}
$$

where $\ell_{O}$ is the thickness of the cadmium filter in mils, and $\mathrm{E}$ in $\mathrm{eV}$.

More resonance terms have to be included in the transmission factor calculation for sm-filter. In the present evaluation, the input parameters of the resonances involved are listed in Table 12 .

Presented in Table 13 are the relative fission rates 
TABLE 12

Neutron Capture Resonance Parameters of

Samarium

$E_{0}(e V)$

$\Gamma(\mathrm{mV})$

$\Gamma_{n}(m V)$

$\Gamma_{\gamma}(\mathrm{mV})$

$\mathrm{J}$

$147 \mathrm{Sm} \mathrm{(158)}$

$\begin{array}{rcccc}3.4 & 62 \pm 5 & 0.94 \pm 0.06 & 61 \pm 5 & 3 \\ 18.3 & 107 \pm 20 & 66 \pm 7 & 41 \pm 17 & 4\end{array}$

$149 \mathrm{Sm} \mathrm{(13.88)}$

$\begin{array}{lllll}0.0976 & 63.5 \pm 1.0 & 0.47 \pm 0.03 & 63 \pm 1.0 & 4\end{array}$

$\begin{array}{lllll}0.873 & 60 \pm 1 & 0.73 \pm 0.05 & 59.8 \pm 1.0 & 4\end{array}$

$\begin{array}{lllll}4.98 & 68.7 \pm 3.0 & 2.0 \pm 0.2 & 66.7 \pm 3.0 & 4\end{array}$

9.0

$74 \pm 8$

$7 \pm 3$

$66.6 \pm 6.0$

4

Bound level

$152 \mathrm{Sm} \mathrm{(26.78)}$
8.03
$201 \pm 8$
$130 \pm 5$
$71 \pm 10$ 
TABLE 13

Relative Fission Rates of Low Energy Resonance in the Thermal, epi-Cd and epi-Sm Fissions of ${ }^{239} \mathrm{Pu}$ *

\begin{tabular}{|c|c|c|c|c|c|c|}
\hline \multirow{2}{*}{$\begin{array}{c}\text { Resonance } \\
\text { Energy } \\
\text { (ev) }\end{array}$} & \multicolumn{2}{|c|}{ Thermal Fission } & \multicolumn{2}{|c|}{ Epi-Cd Fission } & \multicolumn{2}{|c|}{ Epi-Sm Fission } \\
\hline & $\begin{array}{l}\text { Relative } \\
\text { Rate }\end{array}$ & 8 & $\begin{array}{l}\text { Relative } \\
\text { Rate }\end{array}$ & 8 & $\begin{array}{l}\text { Relative } \\
\text { Rate }\end{array}$ & 8 \\
\hline-0.26 & 0.112 & 35 & 0.0014 & 3 & 0.006 & 2 \\
\hline 0.298 & 0.225 & 64 & 0.0161 & 30 & 0.240 & 87 \\
\hline 7.9 & 0.00064 & 0.2 & 0.0064 & 12 & 0.0002 & 0.1 \\
\hline 11.0 & 0.0011 & 0.3 & 0.0112 & 21 & 0.011 & 4 \\
\hline 11.9 & 0.00025 & 0.1 & 0.0025 & 5 & 0.0024 & 1 \\
\hline 14.3 & 0.00015 & $<0.1$ & 0.0015 & 3 & 0.0015 & 0.6 \\
\hline 14.7 & 0.00032 & 0.1 & 0.0032 & 6 & 0.0031 & 1.2 \\
\hline 15.5 & 0.00037 & 0.1 & 0.0027 & 5 & 0.0027 & 1 \\
\hline 17.6 & 0.00026 & 0.1 & 0.0026 & 5 & 0.0024 & 1 \\
\hline 22.2 & 0.00028 & 0.1 & 0.0028 & 5 & 0.0028 & 1 \\
\hline 23.9 & 0.000007 & $<0.1$ & 0.00007 & 0.1 & 0.00007 & $<0.1$ \\
\hline 26.2 & 0.00011 & $<0.1$ & 0.0011 & 2 & 0.0011 & 0.4 \\
\hline 27.3 & 0.00001 & $<0.1$ & 0.0001 & 0.2 & 0.0001 & $<0.1$ \\
\hline 32.3 & 0.00001 & $<0.1$ & 0.0002 & 0.4 & 0.0002 & $<0.1$ \\
\hline 35.3 & 0.000001 & $<0.1$ & 0.00002 & $<0.1$ & 0.00002 & $<0.1$ \\
\hline 41.4 & 0.00009 & $<0.1$ & 0.0009 & 1.6 & 0.0009 & 0.3 \\
\hline 44.5 & 0.00005 & $<0.1$ & 0.0005 & 0.8 & 0.0005 & 0.2 \\
\hline 47.6 & 0.00005 & $<0.1$ & 0.0006 & 1.2 & 0.0006 & 0.2 \\
\hline & 0.351 & & 0.0538 & & 0.276 & \\
\hline
\end{tabular}

* Input resonance parameters are taken fron Bollinger et al. (58). 
of the low energy resonances in the thermal, epi-Cd and epi-Sm fissions of ${ }^{239} \mathrm{Pu}$, as obtained from the above manipulations. We note that several authors (51), (54), (58) have suggested that for the $0.297 \mathrm{eV}$ resonance-neutron fission of ${ }^{239} \mathrm{Pu}$, the compound nucleus should have spin and parity of $1^{+}$, which is different from the "negative energy" resonant states formed in higher proportion in thermal neutron fission. The results of Regier et al. (46) indicated that most of the fissions with epi-Sm neutrons were attributed to the $0.297 \mathrm{eV}$ resonance and they found the prominent variation of the peakto-valley mass-yield ratios for epi-sm neutron compared with thermal fission was only slightly less than the effect observed for monochromatic neutrons of $0.297 \mathrm{eV}$.

B. Mass Yield Variations of Asymmetric fissions

Experimental results (Table 7) from careful nondestructive $\mathrm{Ge}(\mathrm{Li})$ spectrometric measurements indicate that the mass-yield ratios of the asymmetric fission products for both thermal to epi-Cd and thermal to epi-Sm fissions of ${ }^{239} \mathrm{Pu}$ remained constant within the limits of statistical errors. Due to the very high thermal neutron capture cross-section, the loss in the yield of certain isotope such as ${ }^{135} \mathrm{Xe}\left(\sigma_{c}=\right.$ $2.7 \times 10^{6}$ barns) has been estimated with the method given in Appendix A. 
Considering the difference in the fission rates of the low energy resonances of the three types of fission (Table 13), these results suggest that the relative yields of the asymmetric fission products do not vary as a function of the spin state of the fissioning compound nucleus. The upper limit of any such effect is estimated to be $<58$ which is attributed to systematic error of the present measurement. As a matter of fact, this is in agreement with the assumption implied in Wheeler's ${ }^{(51)}$ extension of Bohr's theory ${ }^{(24)}$ in accounting for the variations in the mass-yield ratios of the asymmetric to symmetric fission products.

These results are also consistentwith the two-mode of fission hypothesis, since the relative yields of fission products within the "asymmetric mode" showed no significant fluctuations from one type of fission compared with the other.

It has been mentioned in Chapter 1 that Popa et al. have found significant fluctuations in the relative yields of the asymmetric products from fissions of ${ }^{235} \mathrm{U}$ induced by thermal and epi-cd neutrons. According to these authors, the relative yields of ${ }^{105} \mathrm{Ru}$ and ${ }^{131} \mathrm{I}$ were much higher ( 20\%) for epi-cd fission than for thermal neutron fission, while most of the other nuclides they studied showed less significant changes (Table 14). It seems unlikely that this can be accounted for in terms of shejl structure preference; and it is also impossible to relate the result with the observed variations 
TABLE 14

Activity Ratios of Fission Products for Thermal to epi-Cd Fission of ${ }^{235} \mathrm{U}$ from Nondestructive $\mathrm{Ge}$ (Li) Measurement by Popa et al. (63)

Nuclide

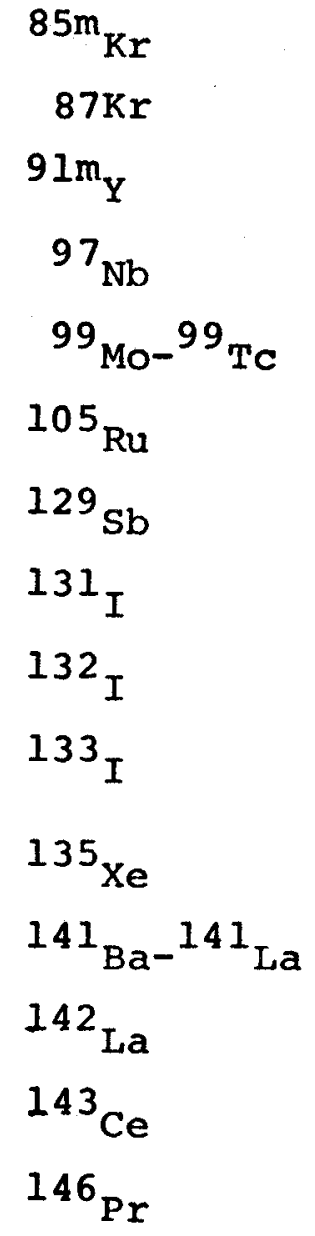

$$
E_{\gamma}(\mathrm{keV})
$$

Ratio of Activities

$$
\left(A_{\text {epi-Cd }} / A_{t h}\right)
$$


in the mass-yield ratios of asymmetric to symmetric fission products (e.g. results of Faler and Tromp ${ }^{(45)}$ ) using Wheeler's extension of A. Bohr's theory. If such effect was due to the difference of spin states of the compound nucleus at the saddle point, the fluctuations would probably be much larger for ${ }^{239} \mathrm{Pu}$ than for ${ }^{235} \mathrm{U}$. In actual fact, the results of the present work on ${ }^{239} \mathrm{Pu}$ exclude such possibility.

c. Mass Yield Variations of Symmetric and Very Asymmetric Fissions

Before discussing the mass yield variations of the symmetric and very asymmetric fissions there are two points needed to be taken into consideration. First, the fractional chain yields of the radiochemically separated nuclides of ${ }^{72} \mathrm{Zn},{ }^{77} \mathrm{Ge},{ }^{77} \mathrm{As},{ }^{112} \mathrm{Pd},{ }^{115} \mathrm{Cd}$ and ${ }^{166} \mathrm{Dy}-{ }^{166} \mathrm{Ho}$ of the respective mass chains have to be evaluated. Results of the estimation based on the method given in Appendix $B$ showed that the yields of these nuclides account for over 998 of the chain yields of the respective chain. Second, because of the high thermal capture cross-section of 139-min ${ }^{165}$ Dy (4700 barns), contribution to the total yield of ${ }^{166}$ Dy from ${ }^{165}$ Dy $(n, \gamma)$ has also been estimated based on method in Appendix A. This was found to be negligible.

It has been found that the relative mass yields of asymmetric fission products do not vary significantly for thermal, epi-Cd and epi-Sm fissions of ${ }^{239} \mathrm{Pu}$. On this basis, 
mass yield variations of some symmetric and very asymmetric fission products were observed. The results are summarized in Table 15 together with experimental data of similar measurements obtained by other authors .

We see that the yield of ${ }^{115} \mathrm{Cd}$ from near symmetric fission of ${ }^{239} \mathrm{Pu}$ decreased by $46 \pm 28$ in epi-sm fission as compared with thermal fission. This is somewhat less than the value reported by Regier et al. ${ }^{(46)}$ in which they found a decrease of $60 \%$. The difference is probably due to the fact that Regier et al. had used metallic samarium shielding which is expected to be a more effective filter in cutting off thermal neutrons. The samarium oxide and cement mixture on the other hand might possibly have "windows" arising from nonuniform packing, and also the thick $\mathrm{Sm}_{2} \mathrm{O}_{3}$-cement $(\sim \mathrm{Im})$ shielding could have certain extent of thermalization effects on the penetrating neutron flux.

A similar effect was observed for ${ }^{112} \mathrm{Pd}$ which showed a decrease of $24 \pm 38$ for the epi-Sm fission compared with thermal fission. Other nuclides in the symmetric region investigated by Regier et al. (46) include ${ }^{121} \mathrm{Sn}$ and ${ }^{125} \mathrm{Sn}$ where they observed decreases of $\sim 558$ and 458 respectively.

In general, the more symmetric fission products showed a larger effect and this became decreasingly smaller for less symmetric fission.

For products from very asymmetric fission, a similar 
Summary of the Mass-Yield Ratios for Thermal to epi-Thermal Fission of ${ }^{239} \mathrm{Pu}$.

\section{(Yield) Thermal}

(Yield) epi-Thermal

Nuclide Ratio This Work odecrease Fritze(49) Croall and Willis (48) Regier et al. (46)

\begin{tabular}{|c|c|c|c|c|c|c|c|}
\hline $72 \mathrm{zn}$ & $\mathrm{Th} / \mathrm{Sm}$ & $1.44 \pm 0.05$ & $30 \pm 2$ & & & & \\
\hline${ }^{72} \mathrm{Zn}$ & $\mathrm{Th} / \mathrm{Cd}$ & & & $1.13 \pm 0.07$ & & & \\
\hline${ }^{77} \mathrm{Ge}$ & $\mathrm{Th} / \mathrm{Sm}$ & $1.08 \pm 0.04$ & & & & & \\
\hline${ }^{77} \mathrm{Ge}$ & $\mathrm{Th} / \mathrm{Cd}$ & $1.07 \pm 0.04$ & & & & & \\
\hline${ }^{77}$ As & $\mathrm{Th} / \mathrm{Cd}$ & $1.03 \pm 0.04$ & & & 0.55 & & \\
\hline${ }^{78}$ As & $\mathrm{Th} / \mathrm{Cd}$ & & & & 0.78 & & \\
\hline${ }^{111} \mathrm{Ag}$ & $\mathrm{Th} / \mathrm{Cd}$ & & & & 1.07 & & \\
\hline $112 \mathrm{Pd}$ & $\mathrm{Th} / \mathrm{Sm}$ & $1.32 \pm 0.08$ & $24 \pm 3$ & & & & \\
\hline $113 \mathrm{Ag}$ & $\mathrm{Th} / \mathrm{Cd}$ & & & & 1.38 & & \\
\hline${ }^{115} \mathrm{Cd}$ & $\mathrm{Th} / \mathrm{Sm}$ & $1.87 \pm 0.08$ & $46 \pm 2$ & & & $2.41 \pm 0.15$ & \\
\hline${ }^{115} \mathrm{Cd}$ & $\mathrm{Th} / \mathrm{Cd}$ & & & $1.42 \pm 0.05$ & & & \\
\hline${ }^{121} \mathrm{Sn}$ & $\mathrm{Th} / \mathrm{Sm}$ & & & & & $2.34 \pm 0.06$ & \\
\hline${ }^{125} \mathrm{Sn}$ & $\mathrm{Th} / \mathrm{Sm}$ & & & & & $1.79 \pm 0.06$ & $\stackrel{6}{a}$ \\
\hline $\begin{array}{l}166_{\mathrm{DY}}- \\
166_{\mathrm{HO}}\end{array}$ & $\mathrm{Th} / \mathrm{Sm}$ & $1.45 \pm 0.07$ & $3 I \pm 2$ & & & & \\
\hline
\end{tabular}


trend of effect was observed in this work for ${ }^{72} \mathrm{zn}$ and ${ }^{166} \mathrm{Ho}$, the two approximately complimentary fragments formed by the fission of $240 \mathrm{Pu}$ compound nucleus. Specifically, the yields of both nuclides were decreased by $30 \pm 2 \%$ for epi-Sm fission as compared with thermal fission.

Only a very slight decrease was observed for the $A=77$ mass chain in the epi-Sm and epi-Cd fissions through careful radiochemical and $\mathrm{Ge}(\mathrm{L} \mathrm{i})$ spectrometric measurements. This is in contradiction with Croall and Willis' investigation (48) in which they reported considerable increases for both ${ }^{77} \mathrm{As}$ and ${ }^{78}$ As in the epi-Ca fission. It is to be noted that in the work of these authors who used end-window $\beta$-counting, any contamination due to $26-\mathrm{hr}{ }^{76}$ As formed by ${ }^{75}$ As $(n, \gamma)$ would be difficult to resolve from the $38.7-\mathrm{hr}{ }^{77} \mathrm{As}$. Furthermore, the specific activity produced in the epi-Sm sample of any contamination of natural arsenic would be higher (resonance integral for activation $=36.8$ barns) than that could be obtained from the thermal sample (thermal neutron activation cross-section $5.4 \pm 1.0$ barns). Hence, any contamination of arsenic would cause an increase in the yield of the epi-Cd sample.

In the present work, the high-resolution Ge(Li) gamma counting technique was employed, and it has been proved experimentally that the contamination of ${ }^{77} \mathrm{Ge}$ due to ${ }^{76} \mathrm{Ge}$ $(n, \gamma)$ is unlikely, especially as an impurity in the aluminum catcher foil. 
(1) Interpretation in terms of two-mode of fission hypothesis The results reported here are in agreement with the two-mode of fission hypothesis of Turkevich and Niday (34), and Fairhall et al. (33), or the so-called "two-component" of Schmitt and Konecny (42) provided one assumes a broad symmetric mode of the gaussian type.

This assumption can be illustrated in Fig. 22 in which the total mass yield curve is represented by the superposition of an asymmetric fission yield curve and a broad symmetric fission yield curve. In order to account for the observed effect in ${ }^{72} \mathrm{Zn}$ and ${ }^{166} \mathrm{Dy}-{ }^{166} \mathrm{Ho}$ similar to the effect in symmetric fission, the width of the gaussian "symmetric mode" was estimated to be $\sim 30$ mass unit (full width at half maximum, FWHM). The analysis of Britt et al. showed an increase in the width of symmetric mode as a function of increasing mass number of the fissioning nucleus. For ${ }^{226} \mathrm{Ra}$ (13MeV p, f), schmitt and Konecny ${ }^{(42)}$ have fitted a "symmetric component" for the triple-peaked mass-yield curve with a gaussian of FWHM of 23 mass unit as compared with the FWHM of 17 mass unit for the purely symmetric fission of ${ }^{209} \mathrm{Bi}$ (22MeV d, f) (see reference (33)).

The invoking of the two-mode hypothesis in explaining the mass yield variations requires two separate saddle points with different densities of states in the channel spectra. Businaro and Gallone (91) and Nosoff (92) argued that quadrupole 


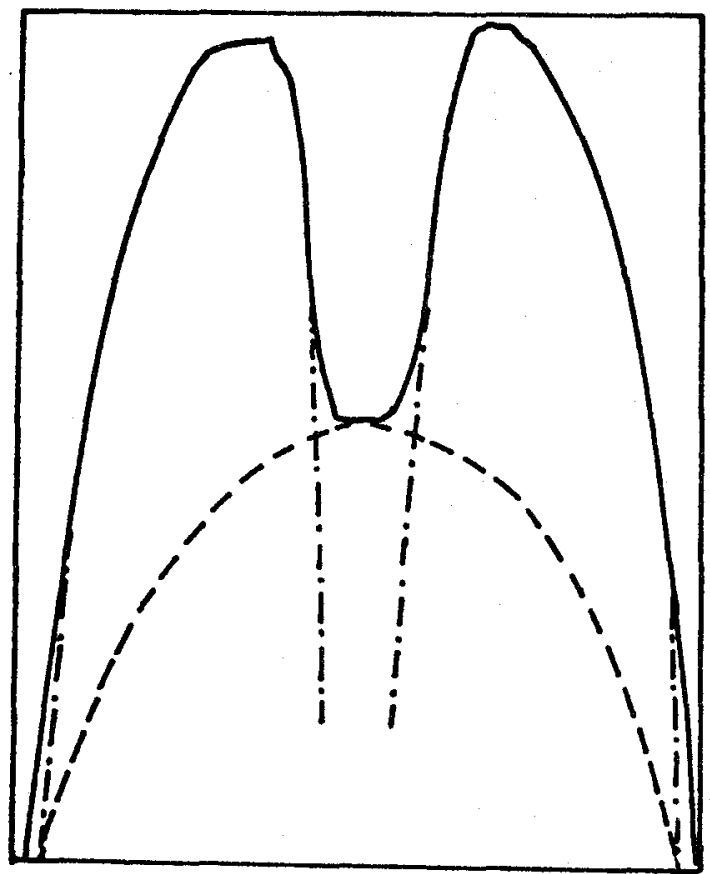

Fig. 22. Decomposition of the fission mass-yield curve (solid line) into two components:

- - "asymmetric component" and

$\longrightarrow-$ "symmetric component". 
deformations might be expected to lead to a saddle point characteristic of symmetric fission while octupole deformations involve a different saddle point which leads to asymmetric fission. The calculation of Johansson (93) shows that by including shell effects with octupole deformations, the energy levels at large deformations are considerably lowered, leading to an enhancement of asymmetric fission.

This complication seems unnecessary for the description of the mechanism of fission because pertinent experiments (angular distribution measurements) have not indicated more than one saddle point. This is avoided by using the "twocomponents" of fission of Schmitt and Konecny (42) in which each component may or may not involve different saddle points. Accordingly, the symmetric component is characteristic of a liquid drop while the asymmetric peaks are described by the fragment-structure. As they observed, since the fragments in the symmetric component are formed in softer, relatively highly deformed configurations, it is possible to have a wider range of fission products.

The broad symmetric mode (or component) assumption is not exclusive as far as the present results are concerned. It is also possible that the observed effect is just a consequence of the broadening of the asymmetric fission mass-yield distribution as the yields of symmetric fission is increased. 
(2) Interpretation in terms of the effect of the transition states of the compound nucleus at the saddle point

The review in Chapter 1 suggests that most of the evidencesconcerning the fission fragment mass, angular and kinetic energy distributions clearly support the channel theory of A. Bohr (24). That is in the process of fission some constraints are maintained in the transition from saddle point to scission.

The asymmetric to symmetric mass-yield ratios have been observed to vary in a systematic manner from resonance to resonance. Resonances are properties of the compound nucleus. Some property of the saddle point compound nucleus, a certain shape parameter in this case, must still be in effect at the point at which the mass distribution is determined. It has been argued that for low energy neutron fission of ${ }^{239} \mathrm{Pu}$, the $0^{+}$resonant states will give rise to a mass-yield distribution with an appreciable amount of symmetric fission, while in the $1^{+}$resonant states symmetric division is forbidden. We also noted that the $0.297 \mathrm{eV}$ resonance might have spin and parity of $\mathrm{I}^{+}$but the negative energy resonance of thermal neutron fission form different states $0^{+}$.

The analysis earlier showed that for thermal fission about $\sim 408$ of the fission events occured in the negative energy resonance and about $\sim 608$ was due to the $0.297 \mathrm{eV}$ resonance. For epiSm fission, negative energy resonance fission contributes very little 
while the $.297 \mathrm{eV}$ resonance fission predominates (85\%), with the rest due to other resonances. Hence the observed decreases of the symmetric products, $46 \%$ in ${ }^{115} \mathrm{Cd}$ and $24 \%$ in ${ }^{112} \mathrm{Pd}$ for epi-Sm fission compared with thermal fission is consistent with the assignments of $\mathrm{O}^{+}$for the negative energy resonance and $\mathrm{I}^{+}$for the $0.297 \mathrm{eV}$ resonance. And the interpretation that fission through the $0^{+}$states has relatively higher yield for symmetric fission than fission through the $1^{+}$states is satisfied. The much smaller fraction of contributions due to other resonances in the epi-thermal region for both thermal and epi-Sm fission is a mixture of the two types of resonant states. The effect of these resonances on the mass yield variations should therefore be small in comparison with the two with lowest energies. This is justified if one takes the experimental data of Fritze (49) on the epi-Cd fission of ${ }^{239} \mathrm{pu}$ into consideration. Table 13 shows roughly the compositions of thermal, epi-Sm and epi-Cd fissions of ${ }^{239} \mathrm{Pu}$ where we see that fissions occur mostly with incident neutron energy greater than $l e v$ in the case with cadmium filter. But the mass-yield variations for ${ }^{115} \mathrm{Cd}$ and ${ }^{72} \mathrm{zn}$ found by Fritze (49) were significantly lower than has been found for epi-Sm fission in this work.

As the mass yields of symmetric fission were decreased, the products from very asymmetric fissions such as $72 \mathrm{zn}$ and ${ }^{166} \mathrm{DY}^{-166}$ Ho were also found lowered by 308 in the epi-Sm 
fission. The decrease in ${ }^{77} \mathrm{Ge}-{ }^{7 / 7}$ As was slight, if any. Presumably, for fission through the $0^{+}$resonant states, the fragments formed in the compound nucleus are soft and highly deformed so that not only symmetric fission is allowed but also very asymmetric fission is probable. For fission passing through the $1^{+}$resonant states, due to the mass-sloshing (pear-shaped) mode of collective vibration, the fragments in the compound nucleus are stiffer because of the preferred configuration of a core corresponding to a double closedshell $(N=82, Z=50)$ being formed in the transition from saddle point to scission; both symmetric fission and very asymmetric fission are forbidden.

If the mass distribution is dependent upon the energy available for deformation and excitation, the spin effect (corresponding to different modes of motion) would imply that the heights of the Coulomb barrier at scission must be different. Moore (94) has pointed out that there are two possible mechanisms for the transition from the saddle point to scission. One of these was the proposition of Blyumkina et al.(95) that the potential energy of collective motion was regarded to be maintained in the transition and finally changed to kinetic energy at scission but not available as deformation energy. The other mechanism viewed the relative separations of the nascent fragments at scission to be different for different types of quadrupole or octupole vibrations. This is 
expected to be reflected in different shapes or curvatures of the fission barriers for the different channels, as deduced by Vandenbosch (96)

Obviously, the present results are better explained consistently in terms of the second mechanism. Accordingly, the separation of the nascent fragments is larger for fission through $0^{+}$resonant states than the separation for fission through the $\mathrm{I}^{+}$states. The fragment kinetic energy is therefore expected to be higher for the latter kind of fission.

The idea lends its support from the results of the measurement of Melkonian and Mehta (53) which showed that the variations of fragment energy in low energy neutron fission of ${ }^{239} \mathrm{Pu}$ is correlated with the J-values. Among the 19 levels they studied, 15 levels were found with high average kinetic energy and 4 levels with lower values. The former being assigned $\mathrm{J}=1$ and the latter $\mathrm{J}=0$ (see Table 2) on the basis of the expected ratio of population of the spin states and also for maximum compatibility with other measurements. The neutron multiplicity measurements of Weinstein et al. (54) also agree with such a mechanism. The average neutron yield per fission $\left(\widetilde{v}_{t}\right)$ in low energy neutron fission of ${ }^{239} \mathrm{pu}$ was found to be higher for fission through the $0^{+}$states than through the $1^{+}$ states.

For fission at higher energies, though the correlation between the compound nucleus spin states and the post-scission 
characteristics was not established (95-97), the above mechanism also seems to hold well. Similar arguments have been invoked by Konecny and schmitt (42) in the study of ${ }^{226} \mathrm{Ra}$ ( $13 \mathrm{MeV} \mathrm{p}, \mathrm{f})$ to describe the "two fission components", as we have discussed earlier. Recently, Burnett et al. have studied the neutron emissions and the total fragment kinetic energies of ${ }^{233} \mathrm{U}(\mathrm{p}, \mathrm{f})$ at proton energies of 13 and $8.5 \mathrm{MeV}$. The increase in fragment excitation energy at a prompt fragment of mass -130 amu was found to be greater than the increase in excitation energy of the compound nucleus. The excess excitation energy wras compensated by a decrease in total fragment kinetic energy. The additional excitation energy was preferentially dissipated to the heavy fragment based on the variations found in the neutron-yield curves. This view also tends to be supported by the fact that in the $13 \mathrm{MeV}$ case, the total fragment kinetic energy is higher at symmetric fission and lower for asymmetric fission, compared with fission induced by $8.5 \mathrm{MeV}$ proton.

\section{Conclusions}

(1) The Ge (Li) spectroscopic measurements indicate that for high-yield products from asymmetric fission, the relative yields do not vary significantly for epi-Cd and epi-Sm fissions of ${ }^{239} \mathrm{Pu}$ as compared with the thermal fission. This is consistent with the assumptions of both the "two-mode" 
(or "two-component") of fission hypothesis, and the extension of the channel theory of A. Bohr often used to interpret the mass-yield variation of symmetric fission as proposed by Wheeler.

(2) The radiochemical measurements observed for epi-Sm fission as compared with thermal fission of ${ }^{239} \mathrm{Pu}$ show (i) marked decreases in the yields of both ${ }^{72} \mathrm{zn}$ and ${ }^{166}$ Ho associated with a large decrease in the yield of ${ }^{115} \mathrm{Cd}$; (ii) the decrease in ${ }^{112} \mathrm{Pd}$ is appreciably less than that of ${ }^{115} \mathrm{Cd}$, the latter being closer to an exact symmetric division of the compound nucleus; (iii) ${ }^{77} \mathrm{Ge}-{ }^{77} \mathrm{As}$, produced in very asymmetric fission, showed a very slight decrease, if any. These results are consistent with the "two-mode" or "two-component" of fission hypothesis. Interpretation in terms of the spin states effect of the compound nucleus at the saddle point is also possible. It is thought that for fission through the $0^{+}$resonant states, the nascent fragments are formed softer and the heavier fragment is relatively highly deformed and so the distance of separation is larger. Symmetric fission is allowed and very asymmetric fission is also possible. In fission through the $1^{+}$resonant states, the nascent fragments formed are stiffer, the heavy fragment probably corresponding to a closed-shell structure of $\mathrm{Z}=50$ and $\mathrm{N}=82$ while the interfragment separation is shorter. This results in the narrow doublepeaked mass distribution. 
APPENDIX A

Correction for Neutron Capture Effect

Because of the high neutron capture cross-sections of certain nucleides formed in fission, it is necessary to estimate the effect on the experimental data obtained in this work. Two most prominent cases were considered in the following.

(i) The loss of the yield of ${ }^{135} \mathrm{Xe}$ due to neutron capture reaction during irradiation:

The formation and disappearance of ${ }^{135} \mathrm{Xe}$ can be expressed as follows:

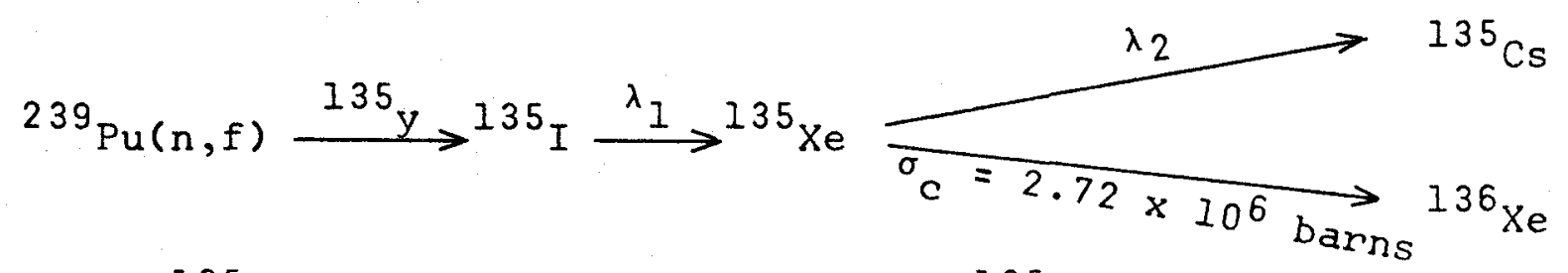

where ${ }^{135} \mathrm{y}$ is the cumulative yield of ${ }^{135} \mathrm{I}$ in fission, $\lambda_{I}$ and $\lambda_{2}$ are decay constants of ${ }^{135} \mathrm{I}$ and ${ }^{135} \mathrm{Xe}$ respectively, and $\sigma_{C}$ is the thermal neutron capture cross-section of ${ }^{135} \mathrm{Xe}$. The rate of formation of ${ }^{135} I$ is given by

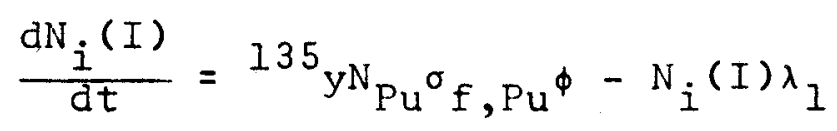

where ${ }^{135} \mathrm{yN}_{\mathrm{Pu}^{\sigma}} \mathrm{f}, \mathrm{Pu}^{\phi}=\mathrm{R}_{\mathrm{f}}{ }^{135} \mathrm{y}=$ rate of production of ${ }^{135} \mathrm{I}$ from fission,

and

$$
\begin{aligned}
& N_{P u}=\text { number of }{ }^{239} \mathrm{Pu} \text { in sample, } \\
& \sigma_{f}=\text { fission cross-section of }{ }^{239} \mathrm{Pu},
\end{aligned}
$$

$\phi=$ thermal neutron flux. 
The rate of formation of ${ }^{135} \mathrm{Xe}$ is given as

$$
\frac{d N_{i}(X e)}{d t}=N_{i}(I) \lambda_{I}-N_{i}(X e) \lambda_{2}-N_{i}(X e) \sigma_{c} \phi
$$

The independent yield of ${ }^{135} \mathrm{Xe}$ from fission was assumed to be negligible based on the estimation made according to Appendix B.

The solutions of differential equations ( 1 ) and

(2) are:

and

$$
N_{i}(I)=\frac{135 y R_{f}}{\lambda_{I}}\left(I-e^{-\lambda_{I} t}\right)
$$

$N_{i}(X e)=\frac{135 y R_{f}}{\lambda_{2}{ }^{+\sigma} c_{c}^{\phi}}\left[1+\frac{\lambda_{2}+\sigma_{c} \phi}{\lambda_{1}-\lambda_{2} 2^{-\sigma} c^{\phi}} e^{-\lambda_{1} t}-\frac{\lambda_{1}}{\lambda_{1}-\lambda_{2} 2^{-\sigma} c^{\phi}} e^{-\left(\lambda_{2}+\sigma_{c} \phi\right) t}\right]$

If $\sigma_{c} \phi=0$,

$$
N_{i}(X e)=\frac{135 y R_{f}}{\lambda_{2}}\left[1+\frac{\lambda_{2}}{\lambda_{1}-\lambda_{2}} e^{-\lambda_{1} t}-\frac{\lambda_{1}}{\lambda_{1}-\lambda_{2}} e^{-\lambda_{2} t}\right]
$$

However, at the time of gamma-ray counting with Ge(Li) detector, a large proportion of ${ }^{135} \mathrm{Xe}$ would have been formed from the decay of ${ }^{135}$ I during the cooling period of the sample through the sequence below,

$$
{ }^{135} \mathrm{I} \stackrel{\lambda_{1}}{\longrightarrow}{ }^{135} \mathrm{Xe} \stackrel{\lambda_{2}}{\longrightarrow}{ }^{135} \mathrm{Cs}
$$

for which

$$
N_{f}(X e)=\frac{\lambda_{1}}{\lambda_{2}-\lambda_{1}} N_{i}(I)\left(e^{-\lambda_{1} t}-e^{-\lambda_{2} t}\right) \text {. }
$$

From the relative values of $\mathrm{N}_{i}(\mathrm{Xe}), \mathrm{N}_{i}^{\prime}(\mathrm{Xe})$ and $\mathrm{N}_{\mathrm{f}}(\mathrm{Xe})$ at the time of radio-counting, the loss of the yield 
of ${ }^{135} \mathrm{Xe}$ due to neutron capture reaction in the thermal fission sample of ${ }^{239} \mathrm{Pu}$ can be corrected and this was $\sim 6 \%$ of the total count rates.

(ii) The contribution of ${ }^{165} \mathrm{Dy}(\mathrm{n}, \gamma)^{166} \mathrm{Dy}$ to the final yield of ${ }^{166}$ Dy. Consider the reactions,

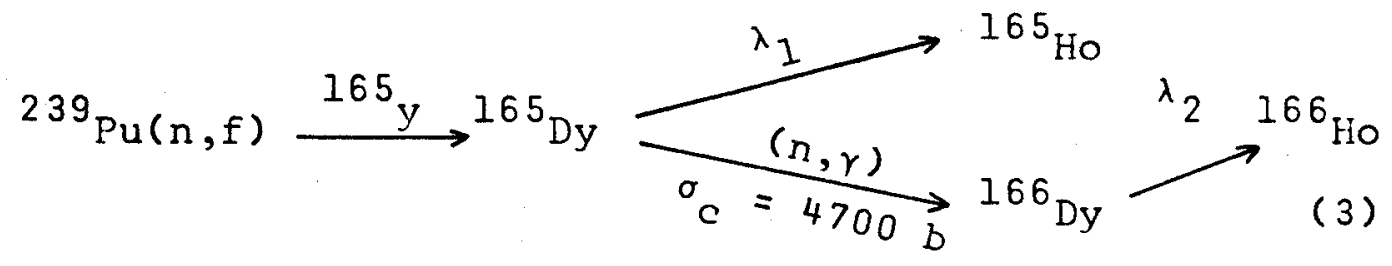

$$
\begin{aligned}
& 239 \mathrm{Pu}(\mathrm{n}, \mathrm{f}) \stackrel{{ }^{166} \mathrm{y}}{\longrightarrow}{ }^{166} \mathrm{Dy} \stackrel{{ }^{\lambda} 2}{\longrightarrow}{ }^{166} \mathrm{HO}
\end{aligned}
$$

and

for which the following differential equations are obvious,

$$
\begin{aligned}
& \frac{d N_{f}(165)}{d t}=165 y R_{f}-N_{f}(165) \lambda_{I}-N_{f}(165) \sigma_{c} \phi \\
& \frac{d N_{c}(166)}{d t}=N_{f}(165) \sigma_{c} \phi-N_{c}(166) \lambda_{2} \\
& \text { and } \quad \frac{d N_{f}(166)}{d t}=166 y R_{f}-N_{f}(166) \lambda_{2}
\end{aligned}
$$

where subscripts ' $c$ ' and ' $f$ ' refer to capture and fission contributions, while the symbols have usual significance as in case ( $i)$. The solutions are given as:

and

$$
\begin{aligned}
& N_{f}(165)=\frac{165 y R}{\lambda_{I}+\sigma_{c} \phi}\left[1-e^{-\left(\lambda_{I}+\sigma_{c} \phi\right) t}\right] \\
& N_{f}(166)=\frac{166 y R}{\lambda_{2}}\left[1-e^{-\lambda_{2} t}\right] \\
& N_{c}(166)=\frac{\sigma_{c}{ }^{\phi 165} y R_{f}}{\lambda_{I}+\sigma_{c}{ }^{\phi}}\left[\frac{1}{\lambda_{2}}+\frac{e^{-\left(\lambda_{I}+\sigma_{c} \phi\right) t}}{\lambda_{I}+\sigma_{c} \phi-\lambda_{2}}\right.
\end{aligned}
$$




$$
\left.-\frac{\lambda_{1}+\sigma_{c} \phi}{\lambda_{2}\left(\lambda_{1}+\sigma_{c}^{\phi-\lambda_{2}}\right)} e^{-\lambda_{2} t}\right]
$$

By substitution of the appropriate values, the ratio of ${ }^{166}$ Dy formed by fission to that formed by neutron capture of ${ }^{165}$ Dy after an irradiation period of 20 hours is $\frac{N_{f}(166)}{N_{c}(166)} \approx 1 / 400$. 
APPENDIX B

Calculation of the Fractional Chain Yield

Approximate fractional chain yields can be obtained by the method of Coryell et al. (99). These authors assumed that the equal charge displacement hypothesis and the wellestablished shape of the charge distribution curve along any isobaric sequence for the thermal fission of ${ }^{235} \mathrm{U}$ are generally valid for fissions differing in compound nucleus and excitation energy.

The following expression which uses the $Z_{p}$ (most probable charge) function of the thermal fission of ${ }^{235} \mathrm{U}$ as reference, was derived for computing, the value of $Z_{p}$ of any mass number A for the fission of a compound nucleus with atomic number $Z_{c}$, and mass number $A_{c}$ :

$$
\begin{gathered}
Z_{p}(A)-Z_{p}(A)^{235}=\Delta Z_{p}(A) \\
\Delta Z_{p}(A)=\frac{1}{2}\left(Z_{c}-92\right)-0.21\left(A_{c}-236\right)+0.19\left(v_{t}-v_{t}^{235}\right)
\end{gathered}
$$

where $\nu_{t}$ is the average number of neutrons emitted per fission. With $v_{t}^{235}=2.43$ and $v_{t}\left({ }^{239} \mathrm{Pu}\right)=2.87^{(5)}$, the $\mathrm{Z}_{\mathrm{P}}$ function for the thermal fission of ${ }^{239} \mathrm{Pu}$ is given as:

$$
Z_{p}(A)=Z_{p}(A)^{235}+0.32 .
$$

The fractional chain yield of a specific nuclide with atomic number $Z$ of an isobaric sequence is then 
evaluated by applying the empirical charge dispersion formula:

$$
P(Z)=(1 / \sqrt{c \pi}) \exp \left(-\left(Z-Z_{p}\right)^{2} / c\right)
$$

where $c=0.80 \pm 0.14$ according to a recent publication of Wahl et al. (9). This Gaussian distribution is normalized so that the sum of all points taken at unit intervals is unity

Cumulative fractional chain yield of a nuclide $(Z, A)$ is obtained by summing the fractional chain yields of itself and its precursors. In this way, using the $\mathrm{z}_{\mathrm{p}}(\mathrm{A})^{235}$ values compiled by Wahl et al. ( 9 ), the cumulative fractional chain yields of ${ }^{72} \mathrm{Zn},{ }^{77} \mathrm{As},{ }^{112} \mathrm{Pd},{ }^{115} \mathrm{Cd}$ and ${ }^{166}$ Dy were found to represent over $99 \%$ of the total yields of the respective mass chain. The sum of the fractional chain yields of ${ }^{77} \mathrm{Ge}$ and $77 \mathrm{~m}_{\mathrm{Ge}}$ also amounts over $99 \%$. 


\section{REFERENCES}

1. 0. Hahn and F. Strassmann, Naturwiss. 27, 11,89 (1939).

2. L. Meitner and O. R. Frisch, Nature 143, 239,471 (1939).

3. N. Bohr and J. A. Wheeler, Phys. Rev. 56, 426 (1939).

4. C. D. Coryell and N. Sugarman (editors), "Radiochemical Studies: The Fission Products". Nat. Nucl. Inergy Ser., Division IV, Plutonium Project Record, Vol. 9 (New York: McGraw-Hill Book Company, 1951).

5. E. K. Hyde, "The Nuclear Properties of the Heavy Elements", Vol. III: Fission Phenomena. PrenticeHall, Englewood Cliffs, New Jersey (1964).

6. J. C. D. Milton and J. S. Fraser, "Nuclear Fission", Ann. Rev. Nucl. Sci. (1966).

7. J. E. Gindler and J. R. Huizenga, "Nuclear Chemistry", Vol. II, p. 2, L. Yaffe (Editor), Academic Press (1968).

8. L. E. Glendenin, C. D. Coryell and R.R. Edwards, Distribution of Nuclear Charge in Fission, Paper 52 in Reference (4).

9. A. C. Wahl, A. E. Norris, R. A. Rouse and J. C. Williams, Proceedings of the Second Symposium on Physics and Chemistry of Fission, Paper IAEA-SM-122/116, Vienna (1969).

10. S. Cohen and W. J. Swiatecki, Ann. Phys. 19, 67 (1962). 22, 406 (1963). 
11. V. M. Strutinskii, N. Ya. Lyashchenko and N. A. Popov, JETP (Sov. Phys.) 16, 418 (1963).

12. D. L. Hill and J. A. Wheeler, Phys. Rev. 89, 1102 (1953).

13. J. R. Nix, Nucl. Phys. Al30, 241 (1969).

14. V. M. Strutinsky, Nucl. Phys. A95, 420 (1965).

15. S. Bjørholm and V. M. Strutinsky, Nucl. Phys. Al36, 1 (1969).

16. P. Fong, Phys. Rev. 102, 434 (1956).

17. J. K. Perring and J. S. Storey, Phys. Rev. 98, 1525 (1955).

18. P. Fong, Phys. Rev. 135, B1338 (1964).

19. W. Brunner and H. Paul, Ann. Physik. 6, 267 (1960).

$$
\begin{aligned}
& \text { 7, } 326 \text { (1961). } \\
& \text { 7, } 333 \text { (1961). } \\
& 8,146 \text { (1961). }
\end{aligned}
$$

20. T. D. Thomas and R. Vandenbosch, Phys. Rev. 133, B976 (1964).

21. R. Ramanna R. Subramanian and R. N. Aiyer, Nucl. Phys. 67, $529(1965)$.

22. L. Wilets, "Theories of Nuclear Fission", Clarendon Press, Oxford (1964).

23. W. J. Swiatecki, Paper SM-60/I, Proceedings of Symposium on Physics and Chemistry of fission, Salzburg, 1965, (Vienna) Vol. 1, p. 3.

24. A. Bohr, Proceedings of the Int. Conf. on the Peaceful Uses of Atomic Energy, Geneva, 1955 (United Nations, New York, 1956), Vol. 2, p. 151. 
25. H. Faissner and K. Wildermuth, Nucl. Phys. 58, 177

$$
\text { (1964). }
$$

26. J. J. Griffin, Proceedings of the Second Symposium on Physics and Chemistry of Fission, Paper IAEA-SM-122/200, IAEA, Vienna (1969).

27. I. Halpern and E. M. Henley, Comments on Nuclear and Particle Physics, V. III, Number 2, 52 (1969).

28. N. Feather, Proceedings of the Second Symposium on Physics and Chemistry of Fission, Paper IAEA-SM-122/201, Vienna (1969).

29. S. Katcoff, Nucleonics 18, 201 (1960).

30. H. G. Thode and R. L. Graham, Can. J. Research 25A, I (1947).

R. K. Wanless and H. G. Thode, Can. J. Phys. 33, 541 (1955).

H. Farrar and R. H. Tomlinson, Can. J. Phys. 40, 943 (1962).

31. R. B. Levy, H. G. Hicks, W. E. Nervik, P. C. Stevenson, J. B. Niday and J. C. Armstrong, Jr., Phys. Rev. 124, 544 (1961).

32. N. I. Borisova, S. M. Dubrovia, V. I. Novgorodtseva, V. A. Pchelin, V. A. Shigin and V. M. Shubko, Sov. J. of Nucl. Phys. (English) $\underline{6}, 331$ (1967).

33. A. W. Fairhall, R. C. Jensen and E. F. Neuzil, paper P/677, Proc. of the 2nd U.N. Conf. on the Peaceful Uses of Atomic Energy 15, Geneva (1958). 
34. A. Turkevich and J. B. Niday, Phys. Rev. 84, 52 (1951).

35. L. J. Colby, M. La Salle-Shouf and J. Cobble, Phys. Rev. 121, 1415 (1961).

36. R. H. Iyer et al., J. Inorg. Nucl. Chem. 25, 465 (1963).

37. H. C. Britt and S. L. Whetstone, Jr., Phys. Rev. 133, B603 (1964).

H. C. Britt, H. E. Wegner and J. C. Gursky, Phys. Rev. $129,2239(1963)$.

38. G. P. Ford, Phys. Rev. 118, 1261 (1960).

39. H. G. Hicks, H. B. Levy, W. E. Nervik, P. C. Stevenson, J. B. Niday and J. C. Armstrong, Jr., Phys. Rev. 128, 700 (1962).

40. G. P. Ford and R. B. Leachman, In "Physics and Chemistry of Fission", Vol. I, p. 333, IAEA, Vienna (1965).

4I. G. R. Choppin and E. F. Meyer, Jr., J. Inorg. Nucl. Chem. 28, 1509 (1966).

42. E. Konecny and H. W. Schmitt, Phys. Rev. 172, 1213 (1968).

43. J. R. Nix and W. J. Swiatecki, Nucl. Phys. 71, I (1965). 44. Los Alamos Radiochemistry Group, Phys. Rev. 107, 325 (1957).

45. K. T. Faler and R. L. Tromp, Phys. Rev. 131, 1746 (1963).

46. R. B. Regier, W. H. Burgus, R. L. Tromp and B. H. Sorenson, Phys. Rev. 119, 2017 (1960). 
47. G. A. Cowan, B. P. Bayhurst and R. J. Prestwood, Phys. Rev. 130,2380 (1963).

G. A. Cowan, B. P. Bayhurst, R. J. Prestwood, J. S. Gilmore and G. W. Knobeloch, Phys. Rev. 144, 979 (1966). Phys. Rev. C 2,615 (1970).

48. J. F. Croall and H. H. Willis, In "Physics and Chemistry of Fission", Vol. I, P. 355, IAEA, Vienna (1965).

49. K. Fritze, Radiochim. Acta $\underline{5}, 57$ (1966).

50. J. A. Wheeler, Physica 22, 1103 (1956). , In "Fast Neutron Physics" (J. B. Marion and J. L. Fowler, eds.) Part 2, p. 2051, Interscience, New York (1963).

51. J. E. Lynn, "Theory of Neutron Resonance Reactions", Clarendon Press, Oxford, 1968.

52. J. J. Griffin, In "Physics and Chemistry of Fission", Vol. I, P. 23, IAEA, Vienna (1965).

53. E. Melkonian and G. K. Mehta, In "Physics and Chemistry of Fission", Vol. II, p. 355, IAEA, Vienna (1965).

54. S. Weinstein, R. Reed and R. C. Block, "Proceedings of the Second Symposium on Physics and Chemistry of Fission", p. 477, IAEA, Vienna (1969).

55. M. Asghar, A. Michaudon, and D. Payer, Phys. Lett. 26B, $664(1964)$.

56. H. Weigman, J. Winter and M. Heske, Nucl. Phys. Al34, 
57. C. D. Bowman, J. Berman and R. Bagham, WASH-1127, Comp. by M. S. Moore (1969).

58. L. M. Bollinger, R. E. Cote and G. E. Thomas, In "Proceedings of the Second Int. Conf. on the Peaceful Uses of Atomic Energy", Vol. 15, p. 127, United Nations, Geneva ( 1958 ).

59. G. D. Sauter and C. D. Bowman, Phys. Rev. Lett. 15, $761(1965)$.

60. T. J. King and R. C. Block, Nucl. Phys. Al38, 556 (1969).

61. M. Asghar, Nucl. Phys. 198, 33 (1967).

62. J. A. Farrell, Phys. Rev. 165, 1371 (1968).

63. P. Popa, M. De. Coster and P. H. M. Van Assche, Nucl. Sci. Eng. 39, 50 (1970).

64. G. E. Gordon, J. W. Harvey and H. Nakahara, Nucleonics 24, 62 (1966).

65. L. Wish, Phys. Rev. 172, 1262 (1968).

66. G. M. Lederer, J. M. Hollander and I. Perlman, "Table of Isotopes", sixth edition, John Wiley $\varepsilon$ Sons (1967).

67. A. Ng, Phys. Rev. 176, 1329 (1968).

68. D. J. Gorman, Ph.D. Thesis, McMaster University (1967).

69. J. Korkisch, "Modern Method for the Separation of Rarer Metal Ions", Oxford, New York, Pergamon Press (1969).

70. J. L. Ryan and E. J. Wheelwright, Proceedings of 2 nd Int. Conf. on the Peaceful Uses of Atomic Energy, United Nations, Geneva (1958), Vol. 17, p. 137. 
71. I. M. Kolthoff and P. J. Elving (editors), "Treatise on Analytical Chemistry", N.Y., Part II, Vol. 9, p. 283 (1962).

72. H. G. Hicks, The Radiochemistry of Zinc, NAS-NS3015 $(1960)$.

73. 0. T. Hogdahl, The Radiochemistry of Palladium, NASNS3052 (1961).

74. J. De Voe, The Radiochemistry of Cadmium, NAS-NS3001 $(1960)$.

75. H. C. Beard, The Radiochemistry of Arsenic, NAS-NS 3002 $(1960)$.

J. A. Marinsky, The Radiochemistry of Germanium, NASNS3043 (1961).

I. F. Croall and H. H. Willis, AERE-R4723 (1964).

76. W. F. Hillebrand, G. E. F. Lundell, H. A. Bright and J. I. Hoffman, "Applied Inorganic Analysis", John Wiley $\varepsilon$ Sons, New York (1953).

77. P. C. Stevenson and W. E. Nervik, The Radiochemistry of the Rare Earths, Scandium, Yttrium and Actinium, NAS-NS3020 (1961).

78. G. R. Choppin and R. I. Silv,, J. Inc g. Nucl. Chem. $\underline{3}, 153$ (1956).

79. H. L. Smith and D. C. Hoffman, J. Inorg. Nucl. Chem. 3, $243(1956)$.

80. J. Minzewski and R. Dybezinski, J. of Chromatog. ?, 98 (1962). 
8I. I. P. Alimarin, Yu. V. Jekolev, A. Z. Miklishanky, N. N. Dogadkin and 0. V. Stepanets, J. Radioanal. Chem. I, 139 (1968).

82. K. Wolfsberg, Anal. Chem. 34, 518 (1962).

83. W. W. Wendlandt, Anal. Chem. 31, 408 (1959).

84. R. C. Vickery, "Analytical Chemistry of the Rare Earths", Oxford, New York, Pergamon Press (1961).

85. R. L. Heath, "Modern Trends in Activation Analysis", J. R. De Voe (Editor), NBS Special publ. 312, Vol. II, p. 959 (1969).

86. J. G. Cuninghame, K. Fritze, J. E. Lynn and C. B. Webster, Nucl. Phys. 84, 49 (1966).

87. C. H. Westcott, CRRP-680 (1957). , AECL-1101 (1962).

88. K. H. Beckurtz and K. Writz, "Neutron Physics", Springer-Verlag, N.Y. (1964).

89. D. J. Hughes and J. A. Harvey, "Neutron Cross-Sections", BNL-325 (1958).

90. R. W. Stoughton and J. Halperin, Nucl. Sci. Eng. 15, $314(1963)$.

91. U. L. Businaro and S. Gallone (1955), Nuovo Cimento I, $629(1955)$.

92. V. G. Nossoff, In "Proc. of the lst Int. Conf. on the Peaceful Uses of Atomic Energy", Vol. 2, p. 205, United Nations, New York (1956).

93. S. A. E. Johansson, Nucl. Phys. 22, 529 (1961). 
94. M. S. Moore, IN-1222 (1969).

95. Yu. A. Blyumkina I. I. Bondarenko, V. F. Kuznetsov, V. G. Nesterov, V. N. Okolovitch, G. N. Smirenkin and L. N. Usachev, Nucl. Phys. 52, 648 (1964).

96. R. Vandenbosch, Nucl. Phys. Al0I, 460 (1967).

97. J. G. Cuninghame, G. P. Kitt and F. R. Rae, Nucl. Phys. 27, 154 (1961).

98. S. C. Burnett, R. L. Ferguson, F. Plasil and H. W. Schmitt, Phys. Rev. C 3, 2034 (1971).

99. C. D. Coryell, M. Kaplan and R. D. Fink, Can. J. Chem. $\underline{39}, 646(1961)$. 